DOI 10.4171/JEMS/410

Gianni Dal Maso

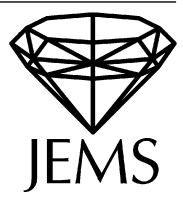

\title{
Generalised functions of bounded deformation
}

Received March 22, 2012 and in revised form December 7, 2012

Abstract. We introduce the space GBD of generalized functions of bounded deformation and study the structure properties of these functions: the rectifiability and the slicing properties of their jump sets, and the existence of their approximate symmetric gradients. We conclude by proving a compactness results for GBD, which leads to a compactness result for the space GSBD of generalized special functions of bounded deformation. The latter is connected to the existence of solutions to a weak formulation of some variational problems arising in fracture mechanics in the framework of linearized elasticity.

Keywords. Free discontinuity problems, special functions of bounded deformation, jump set, rectifiability, slicing, approximate differentiability

\section{Introduction}

The space $\mathrm{BD}(\Omega)$ of functions of bounded deformation was investigated in $[25,31,32,24$, $30]$ to study mathematical models of small strain elasto-plasticity (see also [22, 29, 8, 7]). If $\Omega \subset \mathbb{R}^{n}$ is a bounded open set and $\mathbb{M}^{n \times n}$ denotes the space of $n \times n$-matrices, $\operatorname{BD}(\Omega)$ is the space of functions $u \in L^{1}\left(\Omega ; \mathbb{R}^{n}\right)$ such that the $\mathbb{M}^{n \times n}$-valued distribution $E u$, defined by $(E u)_{i j}:=\frac{1}{2}\left(D_{i} u_{j}+D_{j} u_{i}\right)$, is a bounded Radon measure.

The fine structure of the functions $u \in \mathrm{BD}(\Omega)$ was investigated in [23, 5]. In particular it can be proved that the jump set $J_{u}$ of $u$ is countably $\left(\mathcal{H}^{n-1}, n-1\right)$-rectifiable, where $\mathcal{H}^{n-1}$ is the $(n-1)$-dimensional Hausdorff measure, and that the measure $E u$ can be written as the sum of three measures:

$$
E u=E^{a} u+E^{c} u+E^{j} u,
$$

where $E^{a} u$ is absolutely continuous with respect to the Lebesgue measure $\mathcal{L}^{n}, E^{c} u$ is singular with respect to $\mathcal{L}^{n}$ and satisfies $\left|E^{c} u\right|(B)=0$ for every Borel set $B \subset \Omega$ with $\mathcal{H}^{n-1}(B)<\infty$, while $E^{j} u$ is concentrated on the jump set $J_{u}$. Moreover, if $\mathcal{E} u \in$ $L^{1}\left(\Omega ; \mathbb{M}^{n \times n}\right)$ is the density of $E^{a} u$ with respect to $\mathcal{L}^{n}$, then for $\mathcal{L}^{n}$-a.e $x \in \Omega$ we have (see [5, Theorem 4.3])

$$
\lim _{\rho \rightarrow 0+} \frac{1}{\rho^{n}} \int_{B_{\rho}(x)} \frac{|(u(y)-u(x)-\mathcal{E} u(x)(y-x)) \cdot(y-x)|}{|y-x|^{2}} d y=0,
$$

G. Dal Maso: SISSA, Via Bonomea 265, 34136 Trieste, Italy; e-mail: dalmaso@ sissa.it Mathematics Subject Classification (2010): Primary 49Q20; Secondary 49J45, 26B30, 46E99 
where $B_{\rho}(x)$ denotes the open ball with centre $x$ and radius $\rho$, while the dot denotes the scalar product in $\mathbb{R}^{n}$. Finally, $\mathcal{E} u$ and $J_{u}$ can be reconstructed from the derivatives and the jump sets of the one-dimensional slices of the function $u$ (see [5, Theorem 4.5]).

The space $\operatorname{SBD}(\Omega)$ of special functions of bounded deformation was introduced in [5] and is defined as the space of all functions $u \in \mathrm{BD}(\Omega)$ with $E^{c} u=0$. In the framework of linearized elasticity the variational models for fracture mechanics originated by the seminal paper [21] have a sound mathematical formulation in the space $\operatorname{SBD}(\Omega)$ (see, e.g., $[27,10,13,28,12])$. The common feature of these models is that the main energy term has the form

$$
F_{Q}(u):=\int_{\Omega} Q(\mathcal{E} u) d x+\mathcal{H}^{n-1}\left(J_{u}\right),
$$

where $Q$ is a positive definite quadratic form, which gives the stored elastic energy density as a function of the strain $\mathcal{E} u$.

To prove the existence of solutions to minimum problems related to (1.2) one can use a compactness result proved in [9, Theorem 1.1]: if $u_{k}$ is a sequence in $\operatorname{SBD}(\Omega)$ such that $\left\|u_{k}\right\|_{L^{\infty}\left(\Omega ; \mathbb{R}^{n}\right)}$ and $F_{Q}\left(u_{k}\right)$ are bounded uniformly with respect to $k$, then there exist a subsequence, still denoted by $u_{k}$, and a function $u \in \operatorname{SBD}(\Omega)$, such that $u_{k} \rightarrow u$ pointwise $\mathcal{L}^{n}$-a.e. on $\Omega, \mathcal{E} u_{k} \rightarrow \mathcal{E} u$ weakly in $L^{1}\left(\Omega ; \mathbb{M}^{n \times n}\right)$, and $\mathcal{H}^{n-1}\left(J_{u}\right) \leq \liminf _{k} \mathcal{H}^{n-1}\left(J_{u_{k}}\right)$. The drawback of this result is that it is difficult to obtain a priori bounds of $\left\|u_{k}\right\|_{L^{\infty}\left(\Omega ; \mathbb{R}^{n}\right)}$ for a minimizing sequence, even if lower order terms are present.

A similar difficulty appears also in the study of variational models of fracture mechanics in the framework of finite elasticity (see [16, 17]), whose mathematical formulation uses the function space $\operatorname{SBV}\left(\Omega ; \mathbb{R}^{n}\right)$, for which we refer to [6, Chapter 4]. In these models $\mathcal{E} u$ is replaced by $\nabla u \in L^{1}\left(\Omega ; \mathbb{M}^{n \times n}\right)$, defined for every $u \in \operatorname{SBV}\left(\Omega ; \mathbb{R}^{n}\right)$ as the density of the absolutely continuous part of the measure $D u$ with respect to $\mathcal{L}^{n}$, and the main energy term has the form

$$
F_{W}(u):=\int_{\Omega} W(\nabla u) d x+\mathcal{H}^{n-1}\left(J_{u}\right)
$$

where $W$ is polyconvex and satisfies $W(A) \geq|A|^{2}$ for every $A \in \mathbb{M}^{n \times n}$. The basic compactness theorem for SBV (see [2, 4] and [6, Theorem 4.8]) requires that $\left\|u_{k}\right\|_{L^{\infty}\left(\Omega ; \mathbb{R}^{n}\right)}$ and $F_{W}\left(u_{k}\right)$ are bounded, and an $L^{\infty}$ bound for the minimizing sequences is problematic also in this setting.

In the antiplane case (see [20]), $u$ is a scalar function on $\Omega$ and the $L^{\infty}$ bound is obtained by truncation, assuming that the prescribed boundary values are bounded in $L^{\infty}$. In the vector case, the solution adopted in $[16,17]$ is to formulate the problems in the larger space $\operatorname{GSBV}\left(\Omega ; \mathbb{R}^{n}\right)$, defined as the set of all $\mathcal{L}^{n}$-measurable functions $u: \Omega \rightarrow \mathbb{R}^{n}$ such that $\psi(u) \in \operatorname{BV}_{\text {loc }}\left(\Omega ; \mathbb{R}^{n}\right)$ for every $\psi \in C^{1}\left(\mathbb{R}^{n} ; \mathbb{R}^{n}\right)$ such that $\nabla \psi$ has compact support. For every $u \in \operatorname{GSBV}\left(\Omega ; \mathbb{R}^{n}\right)$ one can define a unique $\mathcal{L}^{n}$-measurable function $\nabla u: \Omega \rightarrow \mathbb{M}^{n \times n}$ such that $\nabla(\psi(u))=\nabla \psi(u) \nabla u \mathcal{L}^{n}$-a.e. in $\Omega$ for every $\psi$ considered above, so that the functional $F_{W}$ can be defined on $\operatorname{GSBV}\left(\Omega ; \mathbb{R}^{n}\right)$.

In this new setting one can rely on the compactness result for GSBV proved in [3] (see also [6, Theorem 4.36]): if $u_{k}$ is a sequence in $\operatorname{GSBV}\left(\Omega ; \mathbb{R}^{n}\right)$ such that $\left\|u_{k}\right\|_{L^{1}\left(\Omega ; \mathbb{R}^{n}\right)}$ and $F_{W}\left(u_{k}\right)$ are bounded uniformly with respect to $k$, then there exist a subsequence, still 
denoted by $u_{k}$, and a function $u \in \operatorname{GSBV}\left(\Omega ; \mathbb{R}^{n}\right)$, such that $u_{k} \rightarrow u$ pointwise $\mathcal{L}^{n}$-a.e. on $\Omega, \nabla u_{k} \rightarrow \nabla u$ weakly in $L^{1}\left(\Omega ; \mathbb{M}^{n \times n}\right)$, and $\mathcal{H}^{n-1}\left(J_{u}\right) \leq \liminf _{k} \mathcal{H}^{n-1}\left(J_{u_{k}}\right)$. An $L^{1}$ bound for a minimizing sequence can be easily obtained from the lower order terms that are usually present in the minimum problems for (1.3).

One may think that the same strategy can be used to formulate and solve the minimum problems for (1.2). The first difficulty in this approach comes from the fact that, if $u \in \operatorname{SBD}(\Omega)$, then, in general, the composite function $\psi(u)$ does not belong to $\operatorname{SBD}(\Omega)$ (it does not even belong to $\operatorname{BD}(\Omega)$ ), unless $\psi(y)=y_{0}+\lambda y$ for some $y_{0} \in \mathbb{R}^{n}$ and $\lambda \in \mathbb{R}$. Therefore a definition of $\operatorname{GSBD}(\Omega)$ that mimics the definition of $\operatorname{GSBV}\left(\Omega ; \mathbb{R}^{n}\right)$ is doomed to failure, since it would not lead to a space containing $\operatorname{SBD}(\Omega)$.

In this paper we propose a different definition of the space $\operatorname{GSBD}(\Omega)$ of generalized special functions of bounded deformation and of the larger space $\operatorname{GBD}(\Omega)$ of generalized functions of bounded deformation. The definition is given by slicing. For every $\xi \in \mathbb{S}^{n-1}=\left\{\xi \in \mathbb{R}^{n}:|\xi|=1\right\}$ let $\Pi^{\xi}:=\left\{y \in \mathbb{R}^{n}: y \cdot \xi=0\right\}$ be the hyperplane orthogonal to $\xi$ passing through the origin. For every set $B \subset \mathbb{R}^{n}$ and for every $y \in \Pi^{\xi}$ we define

$$
B_{y}^{\xi}:=\{t \in \mathbb{R}: y+t \xi \in B\} .
$$

Moreover, for every function $u: B \rightarrow \mathbb{R}^{n}$ we define the function $\hat{u}_{y}^{\xi}: B_{y}^{\xi} \rightarrow \mathbb{R}$ by

$$
\hat{u}_{y}^{\xi}(t):=u(y+t \xi) \cdot \xi
$$

If $u: B \rightarrow \mathbb{R}^{n}$ is $\mathcal{L}^{n}$-measurable, for $\mathcal{H}^{n-1}$-a.e. $y \in \Pi^{\xi}$ the jump set of $\hat{u}_{y}^{\xi}$ is denoted by $J_{\hat{u}_{y}^{\xi}}$. Moreover we set

$$
J_{\hat{u}_{y}^{\xi}}^{1}:=\left\{t \in J_{\hat{u}_{y}^{\xi}}:\left|\left(\hat{u}_{y}^{\xi}\right)^{+}(t)-\left(\hat{u}_{y}^{\xi}\right)^{-}(t)\right| \geq 1\right\}
$$

where $\left(\hat{u}_{y}^{\xi}\right)^{-}(t)$ and $\left(\hat{u}_{y}^{\xi}\right)^{+}(t)$ are the approximate left and right limits of $\hat{u}_{y}^{\xi}$ at $t$.

The space $\operatorname{GBD}(\Omega)$ is defined (see Definition 4.1) as the space of all $\mathcal{L}^{n}$-measurable functions $u: \Omega \rightarrow \mathbb{R}^{n}$ such that there exists a bounded Radon measure $\lambda$ on $\Omega$ with the following property: for every $\xi \in \mathbb{S}^{n-1}$ and for $\mathcal{H}^{n-1}$-a.e. $y \in \Pi^{\xi}$ the function $\hat{u}_{y}^{\xi}$ belongs to $\mathrm{BV}_{\text {loc }}\left(\Omega_{y}^{\xi}\right)$ and

$$
\int_{\Pi \xi}\left(\left|D \hat{u}_{y}^{\xi}\right|\left(B_{y}^{\xi} \backslash J_{\hat{u}_{y}^{\xi}}^{1}\right)+\mathcal{H}^{0}\left(B_{y}^{\xi} \cap J_{\hat{u}_{y}^{\xi}}^{1}\right)\right) d \mathcal{H}^{n-1}(y) \leq \lambda(B)
$$

for every Borel set $B \subset \Omega$. If we replace $\operatorname{BV}_{\text {loc }}\left(\Omega_{y}^{\xi}\right)$ by $\operatorname{SBV}_{\text {loc }}\left(\Omega_{y}^{\xi}\right)$, we obtain the definition of the space $\operatorname{GSBD}(\Omega)$ (see Definition 4.2).

The inclusion $\operatorname{BD}(\Omega) \subset \operatorname{GBD}(\Omega)$ follows from the structure theorem for BD functions (see [5, Theorem 4.5]), while the inclusion $\operatorname{SBD}(\Omega) \subset \operatorname{GSBD}(\Omega)$ follows from $[5$, Proposition 4.7]. Example 12.3 shows that these inclusions are strict.

We prove (see Theorem 6.2) that for every $u \in \operatorname{GBD}(\Omega)$ the approximate jump set $J_{u}$ (see Definition 2.4) is countably $\left(\mathcal{H}^{n-1}, n-1\right)$-rectifiable according to [19, Section 3.2.14] and can be reconstructed from the jump sets of the one-dimensional slices $\hat{u}_{y}^{\xi}$ 
(see Theorem 8.1). More precisely, if $[u]:=u^{+}-u^{-}$is the jump of $u$ on $J_{u}$ (see Definition 2.4), $J_{u}^{\xi}:=\left\{x \in J_{u}:[u](x) \cdot \xi \neq 0\right\}$, and $\left(J_{u}^{\xi}\right)_{y}^{\xi}$ is its slice according to (1.4), then $\left(J_{u}^{\xi}\right)_{y}^{\xi}=J_{\hat{u}_{y}^{\xi}}$ for every $\xi \in \mathbb{S}^{n-1}$ and for $\mathcal{H}^{n-1}$-a.e. $y \in \Pi^{\xi}$.

To prove these results we first study the traces of a function $u \in \operatorname{GBD}(\Omega)$ on a $C^{1}$ submanifold $M$ of $\Omega$ of dimension $n-1$. In this analysis we use the fact that the directional derivative $D_{\xi}(\tau(u \cdot \xi))$ is a bounded Radon measure for every $u \in \operatorname{GBD}(\Omega)$, for every $\xi \in \mathbb{S}^{n-1}$, and every $\tau \in C^{1}(\mathbb{R})$ with $-1 / 2<\tau<1 / 2$ and $0<\tau^{\prime}<1$ (see Theorem 3.5). Then we can apply the result proved in [31, Lemma 1.1] on the traces on $M$ of functions $v \in L^{1}(\Omega)$ such that a single directional derivative $D_{\xi} v$ is a bounded Radon measure on $\Omega$, provided that $\xi$ is transversal to $M$. Inverting $\tau$ we find that the trace of $u \cdot \xi$ is well defined for a set of vectors $\xi$ forming a basis of $\mathbb{R}^{n}$, and this allows us to define the trace of $u$ (see Theorem 5.2).

In the proof of the rectifiability of $J_{u}$ the measure $|\mathcal{E} u|$ used in [5] is replaced by the measure $\hat{\mu}_{u}$ defined for every Borel set $B \subset \Omega$ by

$$
\hat{\mu}_{u}(B):=\sup _{k} \sup \sum_{i=1}^{k} \hat{\mu}_{u}^{\xi_{i}}\left(B_{i}\right),
$$

where $\hat{\mu}_{u}^{\xi}(B)$ is defined as the left-hand side of (1.5) and the second supremum is over all families $\xi_{1}, \ldots, \xi_{k}$ of elements of $\mathbb{S}^{n-1}$ and over all families $B_{1}, \ldots, B_{k}$ of pairwise disjoint Borel subsets of $B$. We first prove (see Theorem 6.1) that the set

$$
\Theta_{u}:=\left\{x \in \Omega: \limsup _{\rho \rightarrow 0+} \frac{\hat{\mu}_{u}\left(B_{\rho}(x)\right)}{\rho^{n-1}}>0\right\}
$$

is countably $\left(\mathcal{H}^{n-1}, n-1\right)$-rectifiable, following an argument developed in [23]. Then we prove (see Theorem 6.2) that $J_{u} \subset \Theta_{u}$ and $\mathcal{H}^{n-1}\left(\Theta_{u} \backslash J_{u}\right)=0$, using the results on the traces of GBD functions on $C^{1}$ manifolds.

A crucial step in the proof of the slicing result for $J_{u}$ is a difficult technical result (see Theorem 7.1) concerning the jump points of the restriction to hyperplanes of a GBD function. The proof of this result follows the lines of the analogous result for BD functions proved in [5, Theorem 5.1], with $|E u|$ replaced again by $\hat{\mu}_{u}$.

Another result of this paper is the existence, for every $u \in \operatorname{GBD}(\Omega)$, of a symmetric approximate gradient. This is a function $\mathcal{E} u \in L^{1}\left(\Omega ; \mathbb{M}_{\text {sym }}^{n \times n}\right)$, where $\mathbb{M}_{\text {sym }}^{n \times n}$ is the space of symmetric $n \times n$ matrices, such that the following variant of (1.1) holds (see Theorem 9.1 and Remark 2.2):

$$
\lim _{\rho \rightarrow 0+} \frac{1}{\rho^{n}} \int_{B_{\rho}(x)} \psi\left(\frac{|(u(y)-u(x)-\mathcal{E} u(x)(y-x)) \cdot(y-x)|}{|y-x|^{2}}\right) d y=0
$$

for $\mathcal{L}^{n}$-a.e $x \in \Omega$ and for every bounded increasing continuous function $\psi: \mathbb{R} \rightarrow \mathbb{R}$ with $\psi(0)=0$. Moreover we prove that $\mathcal{E} u$ can be reconstructed from the approximate gradients $\nabla \hat{u}_{y}^{\xi}$ of the one-dimensional slices $\hat{u}_{y}^{\xi}$ (see Theorem 9.1): for every $\xi \in \mathbb{S}^{n-1}$ and for $\mathcal{H}^{n-1}$-a.e. $y \in \Pi^{\xi}$ we have $(\mathcal{E} u)_{y}^{\xi} \xi \cdot \xi=\nabla \hat{u}_{y}^{\xi} \mathcal{L}^{1}$-a.e. on $\Omega_{y}^{\xi}$. 
In the last section we prove the following analogue of the compact embedding of $\operatorname{BD}(\Omega)$ into $L^{1}\left(\Omega ; \mathbb{R}^{n}\right)$ (see Theorem 11.1 ): every sequence $u_{k}$ in $\operatorname{GBD}(\Omega)$ satisfying uniform bounds for $\left\|u_{k}\right\|_{L^{1}\left(\Omega ; \mathbb{R}^{n}\right)}$ and for the measures $\hat{\mu}_{u_{k}}^{\xi}$ has a subsequence that converges pointwise $\mathcal{L}^{n}$-a.e. on $\Omega$. A slightly stronger bound implies that the limit function belongs to $\operatorname{GBD}(\Omega)$ (see Corollary 11.2).

For the proof we have to modify the well-known Fréchet-Kolmogorov compactness criterion in $L^{1}$ and to find a new version, based on the behaviour of the one-dimensional slices (see Lemma 10.7). The proof follows the lines of [1, Theorem 6.6]. The main difference is that our assumptions concern only the components $u \cdot \xi$ of $u$ and the corresponding slices in the same direction $\xi$.

Arguing as in the proof of [9, Theorem 1.1], we deduce from these results on GBD $(\Omega)$ the following compactness property for $\operatorname{GSBD}(\Omega)$ (see Theorem 11.3): if $u_{k}$ is a sequence in $\operatorname{GSBD}(\Omega)$ such that $\left\|u_{k}\right\|_{L^{1}\left(\Omega ; \mathbb{R}^{n}\right)},\left\|\mathcal{E} u_{k}\right\|_{L^{2}\left(\Omega ; \mathbb{M}_{\text {sym }}^{n \times n}\right)}$, and $\mathcal{H}^{n-1}\left(J_{u_{k}}\right)$ are bounded uniformly with respect to $k$, then there exist a subsequence, still denoted by $u_{k}$, and a function $u \in \operatorname{GSBD}(\Omega)$, such that $u_{k} \rightarrow u$ pointwise $\mathcal{L}^{n}$-a.e. on $\Omega, \mathcal{E} u_{k} \rightarrow \mathcal{E} u$ weakly in $L^{1}\left(\Omega ; \mathbb{M}_{\text {sym }}^{n \times n}\right)$, and $\mathcal{H}^{n-1}\left(J_{u}\right) \leq \liminf \mathcal{H}_{k} \mathcal{H}^{n-1}\left(J_{u_{k}}\right)$.

Finally, Example 12.3 shows that there exists a sequence in $\operatorname{SBD}(\Omega)$, satisfying the hypotheses of the compactness theorem for $\operatorname{GSBD}(\Omega)$, such that the limit function, which necessarily belongs to $\operatorname{GSBD}(\Omega)$, does not belong to $\operatorname{BD}(\Omega)$.

\section{Notation and preliminary results}

For every $x \in \mathbb{R}^{n}$ the open ball of centre $x$ and radius $\rho$ is denoted by $B_{\rho}(x)$. For every $x, y \in \mathbb{R}^{n}$, we use the notation $x \cdot y$ for the scalar product and $|x|$ for the norm. The $n$-dimensional Lebesgue measure on $\mathbb{R}^{n}$ is denoted by $\mathcal{L}^{n}$, while $\mathcal{H}^{k}$ is the $k$-dimensional Hausdorff measure. We use the standard notation $\mathbb{S}^{n-1}:=\left\{\xi \in \mathbb{R}^{n}:|\xi|=1\right\}$ and $\omega_{n}:=\mathcal{L}^{n}\left(B_{1}(0)\right)$, so that $\mathcal{H}^{n-1}\left(\mathbb{S}^{n-1}\right)=n \omega_{n}$.

If $\mu$ is a Borel measure on a Borel set $E \subset \mathbb{R}^{n}$, its total variation is denoted by $|\mu|$. If $A \subset E$ is a Borel set, the Borel measure $\mu\llcorner A$ is defined by $(\mu\llcorner A)(B):=\mu(A \cap B)$ for every Borel set $B \subset E$. If $U \subset \mathbb{R}^{n}$ is an open set, $\mathcal{M}(U)$ is the space of all Radon measures on $U, \mathcal{M}_{b}(U):=\{\mu \in \mathcal{M}(U):|\mu|(U)<\infty\}$ is the space of all bounded Radon measures on $U$, and $\mathcal{M}_{b}^{+}(U):=\left\{\mu \in \mathcal{M}_{b}(U): \mu(B) \geq 0\right.$ for every Borel set $\left.B \subset U\right\}$ is the space of all nonnegative bounded Radon measures on $U$.

Definition 2.1. Let $A$ be a subset of $\mathbb{R}^{n}$, let $v: A \rightarrow \mathbb{R}^{m}$ be an $\mathcal{L}^{n}$-measurable function, let $x \in \mathbb{R}^{n}$ be such that

$$
\limsup _{\rho \rightarrow 0+} \frac{\mathcal{L}^{n}\left(A \cap B_{\rho}(x)\right)}{\rho^{n}}>0,
$$

and let $a \in \mathbb{R}^{m}$. We say that $a$ is the approximate limit of $v$ as $y$ tends to $x$, and write

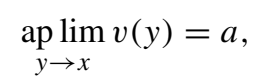

if

$$
\lim _{\rho \rightarrow 0+} \frac{\mathcal{L}^{n}\left(\left\{y \in A \cap B_{\rho}(x):|v(y)-a|>\varepsilon\right\}\right)}{\rho^{n}}=0 \quad \text { for every } \varepsilon>0 .
$$


Remark 2.2. Let $A, v, x$, and $a$ be as in Definition 2.1 and let $\psi$ be a homeomorphism between $\mathbb{R}^{m}$ and a bounded open subset of $\mathbb{R}^{m}$. It is easy to prove that (2.1) holds if and only if

$$
\lim _{\rho \rightarrow 0+} \frac{1}{\rho^{n}} \int_{A \cap B_{\rho}(x)}|\psi(v(y))-\psi(a)| d y=0 .
$$

Definition 2.3. Let $U$ be an open subset of $\mathbb{R}^{n}$. For every $\mathcal{L}^{n}$-measurable function $v: U \rightarrow \mathbb{R}^{m}$ we define the approximate continuity set as the set of points $x \in U$ for which there exists $a \in \mathbb{R}^{m}$ such that

$$
\underset{y \rightarrow x}{\operatorname{ap} \lim } v(y)=a .
$$

The vector $a$ is uniquely determined and is denoted by $\tilde{v}(x)$. The approximate discontinuity set $S_{v}$ is defined as the complement in $U$ of the approximate continuity set.

Definition 2.4. Let $U$ be an open subset of $\mathbb{R}^{n}$. For every $\mathcal{L}^{n}$-measurable function $v: U \rightarrow \mathbb{R}^{m}$ we define the approximate jump set $J_{v}$ as the set of points $x \in U$ for which there exist $a, b \in \mathbb{R}^{m}$, with $a \neq b$, and $v \in \mathbb{S}^{n-1}$ such that

$$
\underset{\substack{(y-x) \cdot v>0 \\ y \rightarrow x}}{\operatorname{ap} \lim } v(y)=a \text { and } \underset{\substack{(y-x) \cdot v<0 \\ y \rightarrow x}}{\operatorname{ap} \lim } v(y)=b .
$$

The triplet $(a, b, v)$ is uniquely determined up to a permutation of $(a, b)$ and a change of sign of $v$, and is denoted by $\left(v^{+}(x), v^{-}(x), v_{v}(x)\right)$. The jump of $v$ is the function $[v]: J_{v} \rightarrow \mathbb{R}^{m}$ defined by $[v](x):=v^{+}(x)-v^{-}(x)$ for every $x \in J_{v}$. Finally, we define

$$
J_{v}^{1}:=\left\{x \in J_{v}:|[v](x)| \geq 1\right\} .
$$

Remark 2.5. It follows easily from the definitions that $J_{v} \subset S_{v}$ for every $\mathcal{L}^{n}$-measurable function $v: U \rightarrow \mathbb{R}^{m}$. Moreover, $\mathcal{L}^{n}\left(S_{v}\right)=0$ and $v=\tilde{v} \mathcal{L}^{n}$-a.e. in $U$ by Remark 2.2 and by Lebesgue's differentiation theorem.

Thanks to Remark 2.2 the next proposition follows from [6, Proposition 3.69].

Proposition 2.6. Let $U$ be an open subset of $\mathbb{R}^{n}$ and let $v: U \rightarrow \mathbb{R}^{m}$ be an $\mathcal{L}^{n}$-measurable function. Then $S_{v}, J_{v}$, and $J_{v}^{1}$ are Borel sets and $\tilde{v}: U \backslash S_{u} \rightarrow \mathbb{R}^{m}$ is a Borel function. Moreover, for every $x \in J_{v}$ we can choose the sign of $v_{v}(x)$ so that $v^{+}: J_{v} \rightarrow \mathbb{R}^{m}$, $v^{-}: J_{v} \rightarrow \mathbb{R}^{m}$, and $v_{v}: J_{v} \rightarrow \mathbb{S}^{n-1}$ are Borel functions.

If $U \subset \mathbb{R}^{n}$ is an open set and $v \in L_{\text {loc }}^{1}(U)$, the gradient $D v$ of $v$ in the sense of distributions is the $\mathbb{R}^{n}$-valued distribution defined by $D v=\left(D_{1} v, \ldots, D_{n} v\right)$. For every $\xi \in \mathbb{R}^{n}$ the directional derivative $D_{\xi} v$ is the distribution $D_{\xi} v:=D v \cdot \xi=\sum_{i} \xi_{i} D_{i} v$. The space $\mathrm{BV}(U)$ of functions of bounded variation is defined as the space of functions $v \in L^{1}(U)$ such that $D_{i} v \in \mathcal{M}_{b}(U)$ for $i=1, \ldots, n$, while $\operatorname{BV}_{\text {loc }}(U)$ is the space of functions $v \in L_{\text {loc }}^{1}(U)$ such that $D_{i} v \in \mathcal{M}(U)$ for $i=1, \ldots, n$. For the properties of BV functions we refer to [18] and [6]. 


\section{Slicing of directional derivatives}

For every $\xi \in \mathbb{R}^{n} \backslash\{0\}$, for every $y \in \mathbb{R}^{n}$, and every set $B \subset \mathbb{R}^{n}$ and we define

$$
B_{y}^{\xi}:=\{t \in \mathbb{R}: y+t \xi \in B\} .
$$

Moreover, for every function $v: B \rightarrow \mathbb{R}^{m}$ we define the function $v_{y}^{\xi}: B_{y}^{\xi} \rightarrow \mathbb{R}^{m}$ by

$$
v_{y}^{\xi}(t):=v(y+t \xi)
$$

When $m=n$, we also consider the function $\hat{v}_{y}^{\xi}: B_{y}^{\xi} \rightarrow \mathbb{R}$ defined by

$$
\hat{v}_{y}^{\xi}(t):=v(y+t \xi) \cdot \xi=v_{y}^{\xi}(t) \cdot \xi .
$$

The hyperplane orthogonal to $\xi$ passing through the origin is denoted by $\Pi^{\xi}:=\left\{y \in \mathbb{R}^{n}\right.$ : $y \cdot \xi=0\}$ and the orthogonal projection from $\mathbb{R}^{n}$ onto $\Pi^{\xi}$ is denoted by $\pi^{\xi}: \mathbb{R}^{n} \rightarrow \Pi^{\xi}$.

Throughout the paper, $\Omega$ is a fixed bounded open subset of $\mathbb{R}^{n}$. The following proposition is proved in [6, Corollary 2.29 and Theorems 3.103 and 3.107] (see also [26]).

Proposition 3.1. Let $v \in L^{1}(\Omega)$ and let $\xi \in \mathbb{R}^{n} \backslash\{0\}$. The following conditions are equivalent:

(a) $D_{\xi} v \in \mathcal{M}_{b}(\Omega)$;

(b) For $\mathcal{H}^{n-1}$-a.e. $y \in \Pi^{\xi}$ the function $v_{y}^{\xi}$ belongs to $\mathrm{BV}\left(\Omega_{y}^{\xi}\right)$ and

$$
\int_{\Pi^{\xi}}\left|D v_{y}^{\xi}\right|\left(\Omega_{y}^{\xi}\right) d \mathcal{H}^{n-1}(y)<\infty .
$$

If these conditions are satisfied, then for every Borel function $g: \Omega \rightarrow \mathbb{R}^{+}$the function

$$
y \mapsto \int_{\Omega_{y}^{\xi}} g_{y}^{\xi} d\left|D v_{y}^{\xi}\right|
$$

is $\mathcal{H}^{n-1}$-measurable on $\Pi^{\xi}$ and

$$
\int_{\Omega} g d\left|D_{\xi} v\right|=\int_{\Pi^{\xi}}\left(\int_{\Omega_{y}^{\xi}} g_{y}^{\xi} d\left|D v_{y}^{\xi}\right|\right) d \mathcal{H}^{n-1}(y) .
$$

Given an open set $U \subset \mathbb{R}$, let $E \subset U$ be $\mathcal{L}^{1}$-measurable with $\mathcal{L}^{1}(U \backslash E)=0$, and let $v: E \rightarrow \mathbb{R}^{m}$ be an integrable function. As $v$ is defined $\mathcal{L}^{1}$-a.e. in $U$, it can be considered as a distribution on $U$, whose derivative is denoted by $D v$. The pointwise variation $(V v)(I)$ of $v$ on an open interval $I \subset U$ is defined by

$$
(V v)(I):=\sup \left\{\sum_{i=1}^{k}\left|v\left(t_{i}\right)-v\left(t_{i-1}\right)\right|: t_{0}<t_{1}<\cdots<t_{k}, t_{i} \in E \cap I\right\} .
$$

We observe that $V v$, unlike $D v$, is sensitive to changes of $v$ (or of the domain of $v$ ) on sets of Lebesgue measure zero. If $(V v)(I)$ is finite, then $D v$ can be represented by a bounded 
measure on $I$ with $|D v|(I) \leq(V v)(I)$. Moreover, if $(V v)(I)<\infty$ for every open interval $I \subset U$, then $V v$ can be extended to a nonnegative Radon measure, still denoted by $V v$, defined on all Borel subsets of $U$. Indeed, if $A \subset U$ is open, we define $(V v)(A)$ as the sum of $(V v)(I)$ over all connected components $I$ of $A$. Then $A \mapsto(V v)(A)$, defined now for all open subsets of $U$, is increasing, inner regular, subadditive and additive on disjoint open sets. Therefore the set function defined for every Borel set $B \subset U$ by

$$
(V v)(B):=\inf \{(V v)(A): A \text { open, } B \subset A \subset U\}
$$

is a Radon measure on $U$, which coincides with (3.4) on all open intervals $I \subset U$ (see, e.g., $[15$, Theorem 14.23]).

Let $v: \Omega \rightarrow \mathbb{R}$ be $\mathcal{L}^{n}$-measurable. By Definition 2.3 for every $\xi \in \mathbb{R}^{n} \backslash\{0\}$ and every $y \in \Pi^{\xi}$ the function $\tilde{v}_{y}^{\xi}$ is defined on $\Omega_{y}^{\xi} \backslash\left(S_{u}\right)_{y}^{\xi}$.

Proposition 3.2. Let $v \in L^{1}(\Omega)$ and let $\xi \in \mathbb{R}^{n} \backslash\{0\}$. Assume that $D_{\xi} v \in \mathcal{M}_{b}(\Omega)$. Then the following conditions are satisfied for $\mathcal{H}^{n-1}$-a.e $y \in \Pi^{\xi}$ :

(a) $\tilde{v}_{y}^{\xi}$ is defined and coincides with $v_{y}^{\xi} \mathcal{L}^{1}$-a.e. on $\Omega_{y}^{\xi}$;

(b) $v_{y}^{\xi} \in \mathrm{BV}\left(\Omega_{y}^{\xi}\right)$ and $\left|D v_{y}^{\xi}\right|(B)=\left(V \tilde{v}_{y}^{\xi}\right)(B)$ for every Borel set $B \subset \Omega_{y}^{\xi}$.

Proof. It is enough to repeat the proof of [5, Proposition 3.2].

We now investigate the behaviour of truncations of scalar functions, and the combined effect of truncation and slicing. The following definition introduces the relevant truncation functions.

Definition 3.3. Let $\mathcal{T}$ be the set of all functions $\tau \in C^{1}(\mathbb{R})$ with $-1 / 2 \leq \tau \leq 1 / 2$ and $0 \leq \tau^{\prime} \leq 1$

The following proposition deals with the one-dimensional case. It provides a bound on the distributional derivative of a function starting from a uniform bound of its truncations.

Proposition 3.4. Let $U$ be a bounded open subset of $\mathbb{R}$, let $v: U \rightarrow \mathbb{R}$ be $\mathcal{L}^{1}$-measurable, and let $\lambda \in \mathcal{M}_{b}^{+}(U)$. Suppose that for every $\tau \in \mathcal{T}$ we have $\tau(v) \in \mathrm{BV}(U)$ and

$$
|D(\tau(v))|(B) \leq \lambda(B) \quad \text { for every Borel set } B \subset U .
$$

Then $v \in \mathrm{BV}_{\mathrm{loc}}(U)$ and

$$
|D v|\left(B \backslash J_{v}^{1}\right)+\mathcal{H}^{0}\left(B \cap J_{v}^{1}\right) \leq \lambda(B) \quad \text { for every Borel set } B \subset U .
$$

If $U$ has a finite number of connected components, then $v \in \mathrm{BV}(U)$.

Proof. It is enough to prove the result when $U$ is a bounded open interval. In this case we have to prove that $v \in \mathrm{BV}(U)$ and that (3.6) holds. Let us fix $\tau_{0} \in \mathcal{T}$ with $\tau_{0}^{\prime}(t)>0$ and $\tau_{0}(-t)=-\tau_{0}(t)$ for every $t \in \mathbb{R}$. Then the function $v_{0}:=\tau_{0}(v)$ belongs to $\operatorname{BV}(U)$. Since $\tau_{0}^{-1}$ is continuous, we have $J_{v}=J_{v_{0}}$. 
For every $a>0$ let $\sigma_{a}$ be the truncation function defined by $\sigma_{a}(t)=-a$ for $t \leq-a$, $\sigma_{a}(t)=t$ for $-a \leq t \leq a$, and $\sigma_{a}(t)=a$ for $t \geq a$. Let us fix an integer $m>0$ and let $v_{m}:=\sigma_{m}(v)$. We claim that $v_{m} \in \operatorname{BV}(U)$ and

$$
\left|D v_{m}\right|\left(B \backslash J_{v_{m}}^{1}\right)+\mathcal{H}^{0}\left(B \cap J_{v_{m}}^{1}\right) \leq \lambda(B)
$$

for every Borel set $B \subset U$. Indeed, since $v_{0} \in \mathrm{BV}(U), v_{m}=\sigma_{m}\left(\tau_{0}^{-1}\left(v_{0}\right)\right)$, and $\sigma_{m}\left(\tau_{0}^{-1}\right)=\tau_{0}^{-1}\left(\tau_{\tau_{0}(m)}\right)$ is Lipschitz continuous on $\mathbb{R}$, we deduce that $v_{m} \in \mathrm{BV}(U)$. By (3.5) and by Vol'pert's chain rule in BV (see [33] and [6, Theorem 3.96]) for every $\tau \in \mathcal{T}$ and every Borel set $B \subset U$ we have

$$
\begin{aligned}
\int_{B \backslash J_{v_{m}}} \tau^{\prime}\left(\tilde{v}_{m}\right) d\left|D v_{m}\right| & =\left|D\left(\tau\left(v_{m}\right)\right)\right|\left(B \backslash J_{v_{m}}\right) \leq|D(\tau(v))|\left(B \backslash J_{v_{m}}\right) \\
& \leq \lambda\left(B \backslash J_{v_{m}}\right),
\end{aligned}
$$

where $\tilde{v}_{m}$ is the precise representative introduced in Definition 2.3. Note that $\tilde{v}_{m}(t)$ is defined for every $t \in U \backslash J_{v_{m}}$ by well known properties of BV functions in dimension one.

For every integer $k$ there exists a function $\tau_{k} \in \mathcal{T}$ such that $\tau_{k}(t)=t-k / 2$ for $k / 2-1 / 4 \leq t \leq k / 2+1 / 4$. Thus (3.8) gives

$$
\left|D v_{m}\right|\left(B_{k}\right) \leq \lambda\left(B_{k}\right),
$$

where $B_{k}:=\left\{t \in B \backslash J_{v_{m}}: k / 2-1 / 4<\tilde{v}_{m}(t) \leq k / 2+1 / 4\right\}$. Summing over $k$ we obtain

$$
\left|D v_{m}\right|\left(B \backslash J_{v_{m}}\right) \leq \lambda\left(B \backslash J_{v_{m}}\right) .
$$

Let us fix $t \in J_{v_{m}} \subset J_{v}$. By (3.5) for every $\tau \in \mathcal{T}$ we have

$$
\left|\tau\left(v^{+}(t)\right)-\tau\left(v^{-}(t)\right)\right| \leq \lambda(\{t\})
$$

If $t \in J_{v_{m}} \backslash J_{v_{m}}^{1}$, there exists $\tau \in \mathcal{T}$ such that $\left|\left[v_{m}\right](t)\right|=\left|\tau\left(v_{m}^{+}(t)\right)-\tau\left(v_{m}^{-}(t)\right)\right| \leq$ $\left|\tau\left(v^{+}(t)\right)-\tau\left(v^{-}(t)\right)\right|$. This implies $\left|\left[v_{m}\right](t)\right| \leq \lambda(\{t\})$, hence

$$
\left|D v_{m}\right|(\{t\}) \leq \lambda(\{t\}) \quad \text { for every } t \in J_{v_{m}} \backslash J_{v_{m}}^{1} .
$$

If $t \in J_{v_{m}}^{1}$, for every $\varepsilon>0$ there exists $\tau \in \mathcal{T}$ such that $1-\varepsilon \leq\left|\tau\left(v_{m}^{+}(t)\right)-\tau\left(v_{m}^{-}(t)\right)\right| \leq$ $\left|\tau\left(v^{+}(t)\right)-\tau\left(v^{-}(t)\right)\right|$, which gives

$$
1 \leq \lambda(\{t\}) \quad \text { for every } t \in J_{v_{m}}^{1} \text {. }
$$

Inequality (3.7) follows now from (3.9)-(3.11).

Let $J_{v_{m}}^{1 / 2}:=\left\{t \in J_{v_{m}}:\left|\left[v_{m}\right](t)\right| \geq 1 / 2\right\}$. By (3.10) and (3.11) we have $\mathcal{H}^{0}\left(J_{v_{m}}^{1 / 2}\right) \leq$ $2 \lambda\left(J_{v_{m}}^{1 / 2}\right) \leq 2 \lambda(U)<\infty$. Since $J_{v}^{1}$ is contained in the union of the increasing sequence $J_{v_{m}}^{1 / 2}$, we obtain $\mathcal{H}^{0}\left(J_{v}^{1}\right) \leq 2 \lambda(U)<\infty$. Using (3.7) we obtain

$$
\left|D v_{m}\right|(U) \leq \lambda(U)+\int_{J_{v_{m}}^{1}}\left|\left[v_{m}\right]\right| d \mathcal{H}^{0} \leq \lambda(U)+\int_{J_{v}^{1}}|[v]| d \mathcal{H}^{0}<\infty .
$$


Let us fix $t_{0} \in U \backslash J_{v} \subset U \backslash J_{v_{m}}$. Since $U$ is an interval, we have

$$
\left|\tilde{v}_{m}(t)\right| \leq\left|\tilde{v}_{m}(t)-\tilde{v}_{m}\left(t_{0}\right)\right|+\left|\tilde{v}_{m}\left(t_{0}\right)\right| \leq\left|D v_{m}\right|(U)+\left|\tilde{v}\left(t_{0}\right)\right|
$$

for every $m$ and every $t \in U \backslash J_{v} \subset U \backslash J_{v_{m}}$. By (3.12) this inequality implies

$$
\left\|v_{m}\right\|_{L^{\infty}(U)} \leq \lambda(U)+\int_{J_{v}^{1}}|[v]| d \mathcal{H}^{0}+\left|\tilde{v}\left(t_{0}\right)\right|<\infty .
$$

Since the right-hand side does not depend on $m$, there exists $m_{0}$ such that $\left\|v_{m_{0}}\right\|_{L^{\infty}(U)}$ $<m_{0}$. This implies that $v=v_{m_{0}}$, hence $v \in \mathrm{BV}(U)$ and (3.6) follows from (3.7).

The following theorem is the main result of this section. It connects a uniform estimate on the directional derivatives of the truncations with an estimate on the one-dimensional slices. The equivalence proved in the theorem will be used in the definition of the space $\operatorname{GBD}(\Omega)$.

Theorem 3.5. Let $v: \Omega \rightarrow \mathbb{R}$ be $\mathcal{L}^{n}$-measurable, let $\xi \in \mathbb{R}^{n} \backslash\{0\}$, and let $\lambda \in \mathcal{M}_{b}^{+}(\Omega)$. The following conditions are equivalent:

(a) for every $\tau \in \mathcal{T}$ the partial derivative $D_{\xi}(\tau(v))$ belongs to $\mathcal{M}_{b}(\Omega)$ and its total variation satisfies

$$
\left|D_{\xi}(\tau(v))\right|(B) \leq \lambda(B) \quad \text { for every Borel set } B \subset \Omega ;
$$

(b) for $\mathcal{H}^{n-1}$-a.e. $y \in \Pi^{\xi}$ the function $v_{y}^{\xi}$ belongs to $\mathrm{BV}_{\text {loc }}\left(\Omega_{y}^{\xi}\right)$ and

$$
\int_{\Pi \xi}\left(\left|D v_{y}^{\xi}\right|\left(B_{y}^{\xi} \backslash J_{v_{y}^{\xi}}^{1}\right)+\mathcal{H}^{0}\left(B_{y}^{\xi} \cap J_{v_{y}^{\xi}}^{1}\right)\right) d \mathcal{H}^{n-1}(y) \leq \lambda(B)
$$

for every Borel set $B \subset \Omega$.

The following lemma justifies the integral in (3.14).

Lemma 3.6. Let $v: \Omega \rightarrow \mathbb{R}$ be $\mathcal{L}^{n}$-measurable and let $\xi \in \mathbb{R}^{n} \backslash\{0\}$. Assume that for $\mathcal{H}^{n-1}$-a.e. $y \in \Pi^{\xi}$ the function $v_{y}^{\xi}$ belongs to $\mathrm{BV}_{\text {loc }}\left(\Omega_{y}^{\xi}\right)$. Then for every Borel set $B \subset \Omega$ the function

$$
y \mapsto\left|D v_{y}^{\xi}\right|\left(B_{y}^{\xi} \backslash J_{v_{y}^{\xi}}^{1}\right)+\mathcal{H}^{0}\left(B_{y}^{\xi} \cap J_{v_{y}^{\xi}}^{1}\right)
$$

is $\mathcal{H}^{n-1}$-measurable on $\Pi^{\xi}$.

Proof. By modifying $v$ on a set of Lebesgue measure zero, we may assume that $v$ is a Borel function on $\Omega$ and that $v_{y}^{\xi} \in \mathrm{BV}_{\text {loc }}\left(\Omega_{y}^{\xi}\right)$ for every $y \in \Pi^{\xi}$. For every $x \in \Omega$ we define

$$
v_{+}^{\xi}(x):=\limsup _{\rho \rightarrow 0+} \frac{1}{\rho} \int_{0}^{\rho} v(x+s \xi) d s \quad \text { and } \quad v_{-}^{\xi}(x):=\limsup _{\rho \rightarrow 0+} \frac{1}{\rho} \int_{-\rho}^{0} v(x+s \xi) d s .
$$


By Fubini's theorem $v_{+}^{\xi}$ and $v_{-}^{\xi}$ are Borel functions on $\Omega$. Therefore $F:=\{x \in \Omega$ : $\left.\left|v_{+}^{\xi}(x)-v_{-}^{\xi}(x)\right| \geq 1\right\}$ is a Borel set. For every $y \in \Pi^{\xi}$ we have $\left(v_{+}^{\xi}\right)_{y}^{\xi}=\left(v_{-}^{\xi}\right)_{y}^{\xi}=v_{y}^{\xi}$ $\mathcal{L}^{1}$-a.e. in $\Omega_{y}^{\xi}$ thanks to Lebesgue's differentiation theorem. By elementary properties of $\mathrm{BV}$ functions in dimension one, this implies $J_{v_{y}^{\xi}}^{1}=F_{y}^{\xi}$ for every $y \in \Pi^{\xi}$. Therefore

$$
\left|D v_{y}^{\xi}\right|\left(B_{y}^{\xi} \backslash J_{v_{y}^{\xi}}^{1}\right)+\mathcal{H}^{0}\left(B_{y}^{\xi} \cap J_{v_{y}^{\xi}}^{1}\right)=\left|D v_{y}^{\xi}\right|\left(B_{y}^{\xi} \backslash F_{y}^{\xi}\right)+\mathcal{H}^{0}\left(B_{y}^{\xi} \cap F_{y}^{\xi}\right)
$$

for every Borel set $B \subset \Omega$ and every $y \in \Pi^{\xi}$. The measurability of (3.15) follows now from (3.2) and from the measurable projection theorem (see, e.g., [14, Proposition 8.4.4]).

Definition 3.7. If condition (b) of Theorem 3.5 is satisfied, for $\mathcal{H}^{n-1}$-a.e. $y \in \Pi^{\xi}$ we can define a measure $\mu_{y}^{\xi} \in \mathcal{M}_{b}^{+}\left(\Omega_{y}^{\xi}\right)$ by setting

$$
\mu_{y}^{\xi}(B):=\left|D v_{y}^{\xi}\right|\left(B \backslash J_{v_{y}^{\xi}}^{1}\right)+\mathcal{H}^{0}\left(B \cap J_{v_{y}^{\xi}}^{1}\right) \quad \text { for every Borel set } B \subset \Omega_{y}^{\xi} .
$$

Moreover, by Lemma 3.6 and (3.14) we can define a measure $\mu^{\xi} \in \mathcal{M}_{b}^{+}(\Omega)$ by setting

$$
\mu^{\xi}(B):=\int_{\Pi^{\xi}} \mu_{y}^{\xi}\left(B_{y}^{\xi}\right) d \mathcal{H}^{n-1}(y) \quad \text { for every Borel set } B \subset \Omega .
$$

It follows from condition (b) of Theorem 3.5 that

$$
\mu^{\xi}(B) \leq \lambda(B) \quad \text { for every Borel set } B \subset \Omega \text {. }
$$

Proof of Theorem 3.5. Assume (a). Let $\hat{\mathcal{T}}$ be a countable subset of $\mathcal{T}$ such that for every $\tau \in \mathcal{T}$ there exists a sequence $\tau_{k}$ in $\hat{\mathcal{T}}$ converging to $\tau$ pointwise on $\mathbb{R}$. Let us fix $\tau \in \hat{\mathcal{T}}$ and let $w:=\tau(v)$ and $\omega:=\pi^{\xi}(\lambda)$, where $\pi^{\xi}$ is the orthogonal projection onto $\Pi^{\xi}$. Let $N$ be a Borel subset of $\Pi^{\xi}$, with $\mathcal{H}^{n-1}(N)=0$, such that the singular part of $\omega$ with respect to $\mathcal{H}^{n-1}\left\llcorner\Pi^{\xi}\right.$ is concentrated on $N$, and let $g: \Pi^{\xi} \rightarrow \mathbb{R}^{+}$be the density of the absolutely continuous part of $\omega$ with respect to $\mathcal{H}^{n-1}\left\llcorner\Pi^{\xi}\right.$. By the disintegration theorem (see, e.g., [6, Theorem 2.28]) there exists a Borel measurable family $\left(\lambda_{y}^{\xi}\right)_{y \in \Pi^{\xi}}$ of Radon measures, with $\lambda_{y}^{\xi} \in \mathcal{M}_{b}^{+}\left(\Omega_{y}^{\xi}\right)$, such that

$$
\lambda(B)=\int_{\Pi^{\xi}} \lambda_{y}^{\xi}\left(B_{y}^{\xi}\right) d \omega(y) \quad \text { for every Borel set } B \subset \Omega .
$$

By (a) and Proposition 3.1 the function $w_{y}^{\xi}$ belongs to BV $\left(\Omega_{y}^{\xi}\right)$ for $\mathcal{H}^{n-1}$-a.e. $y \in \Pi^{\xi}$. Given a Borel set $A \subset \Pi^{\xi}$ and an interval $I \subset \mathbb{R}$, by (3.3), (3.13), and (3.20), applied to the set $\left\{y+t \xi: y \in A, t \in I \cap \Omega_{y}^{\xi}\right\}$, we have

$$
\begin{aligned}
\int_{A}\left|D w_{y}^{\xi}\right|\left(I \cap \Omega_{y}^{\xi}\right) d \mathcal{H}^{n-1}(y) & =\int_{A \backslash N}\left|D w_{y}^{\xi}\right|\left(I \cap \Omega_{y}^{\xi}\right) d \mathcal{H}^{n-1}(y) \\
& \leq \int_{A} \lambda_{y}^{\xi}\left(I \cap \Omega_{y}^{\xi}\right) g(y) d \mathcal{H}^{n-1}(y)
\end{aligned}
$$


It follows that for $\mathcal{H}^{n-1}$-a.e. $y \in \Pi_{y}^{\xi}$ we have

$$
\left|D w_{y}^{\xi}\right|(B) \leq g(y) \lambda_{y}^{\xi}(B) \quad \text { for every Borel set } B \subset \Omega_{y}^{\xi} .
$$

Since $w_{y}^{\xi}=\tau\left(v_{y}^{\xi}\right)$, from (3.21) we deduce that

$$
\left|D\left(\tau\left(v_{y}^{\xi}\right)\right)\right|(B) \leq g(y) \lambda_{y}^{\xi}(B) \quad \text { for every Borel set } B \subset \Omega_{y}^{\xi},
$$

hence

$$
\int_{\Omega_{y}^{\xi}} \tau\left(v_{y}^{\xi}\right) D \varphi d t \leq g(y) \lambda_{y}^{\xi}(\operatorname{supp} \varphi)
$$

for every $\varphi \in C_{c}^{1}\left(\Omega_{y}^{\xi}\right)$ with $|\varphi| \leq 1$ on $\Omega_{y}^{\xi}$. Since $\hat{\mathcal{T}}$ is countable, for $\mathcal{H}^{n-1}$-a.e. $y \in \Pi_{y}^{\xi}$ inequality (3.23) holds for all $\tau \in \hat{\mathcal{T}}$. From the density property of $\hat{\mathcal{T}}$ we conclude that for $\mathcal{H}^{n-1}$-a.e. $y \in \Pi_{y}^{\xi}$ inequality (3.23), and hence (3.22), holds for every $\tau \in \mathcal{T}$. Therefore Proposition 3.4 implies that $v_{y}^{\xi} \in \mathrm{BV}_{\mathrm{loc}}\left(\Omega_{y}^{\xi}\right)$ and

$$
\left|D v_{y}^{\xi}\right|\left(B \backslash J_{v_{y}^{\xi}}^{1}\right)+\mathcal{H}^{0}\left(B \cap J_{v_{y}^{\xi}}^{1}\right) \leq g(y) \lambda_{y}^{\xi}(B)
$$

for $\mathcal{H}^{n-1}$-a.e. $y \in \Pi^{\xi}$ and every Borel set $B \subset \Omega_{y}^{\xi}$.

Integrating (3.24) over $\Pi^{\xi}$ we obtain

$$
\begin{aligned}
\int_{\Pi \xi}\left(\left|D v_{y}^{\xi}\right|\left(B_{y}^{\xi} \backslash J_{v_{y}^{\xi}}^{1}\right)+\mathcal{H}^{0}\left(B_{y}^{\xi} \cap J_{v_{y}^{\xi}}^{1}\right)\right) & d \mathcal{H}^{n-1}(y) \\
& \leq \int_{\Pi \xi} g(y) \lambda_{y}^{\xi}\left(B_{y}^{\xi}\right) d \mathcal{H}^{n-1}(y) \leq \lambda(B)
\end{aligned}
$$

where we have used (3.20) in the last line. This concludes the proof of (3.14) and of the implication (a) $\Rightarrow$ (b).

Assume now (b) and let $\mu_{y}^{\xi}$ and $\mu^{\xi}$ be the measures introduced in Definition 3.7. We fix $\tau \in \mathcal{T}$ and we set $w:=\tau(v)$. Then for $\mathcal{H}^{n-1}$-a.e. $y \in \Pi^{\xi}$ the function $w_{y}^{\xi}$ belongs to $\operatorname{BV}\left(\Omega_{y}^{\xi}\right)$. Since $0 \leq \tau^{\prime} \leq 1$ we have $\left|D w_{y}^{\xi}\right|(B) \leq\left|D v_{y}^{\xi}\right|(B)$ for every Borel set $B \subset \Omega_{y}^{\xi}$. Since $\left|\tau\left(\left(v_{y}^{\xi}\right)^{+}\right)-\tau\left(\left(v_{y}^{\bar{\xi}}\right)^{-}\right)\right| \leq 1$ we have also $\left|D w_{y}^{\xi}\right|(B) \leq \mathcal{H}^{0}(B)$ for every Borel set $B \subset J_{v_{y}^{\xi}}$. Using (3.17) we obtain

$$
\begin{aligned}
& \left|D w_{y}^{\xi}\right|\left(B \backslash J_{v_{y}^{\xi}}^{1}\right) \leq\left|D v_{y}^{\xi}\right|\left(B \backslash J_{v_{y}^{\xi}}^{1}\right)=\mu_{y}^{\xi}\left(B \backslash J_{v_{y}^{\xi}}^{1}\right), \\
& \left|D w_{y}^{\xi}\right|\left(B \cap J_{v_{y}^{\xi}}^{1}\right) \leq \mathcal{H}^{0}\left(B \cap J_{v_{y}^{\xi}}^{1}\right)=\mu_{y}^{\xi}\left(B \cap J_{v_{y}^{\xi}}^{1}\right)
\end{aligned}
$$

for every Borel set $B \subset \Omega_{y}^{\xi}$. It follows that $\left|D w_{y}^{\xi}\right|(B) \leq \mu_{y}^{\xi}(B)<\infty$ for $\mathcal{H}^{n-1}$-a.e. $y \in \Pi^{\xi}$ and for every Borel set $B \subset \Omega_{y}^{\xi}$. By Proposition 3.1 and by (3.18) and (3.19) we have $D_{\xi} w \in \mathcal{M}_{b}(\Omega)$ and $\left|D_{\xi} w\right|(B) \leq \mu^{\xi}(B) \leq \lambda(B)$ for every Borel set $B \subset \Omega$. This proves (3.13) and concludes the proof of the implication $(\mathrm{b}) \Rightarrow(\mathrm{a})$.

The following theorem shows that the measure $\mu^{\xi}$, which was defined by slicing, can also be obtained from the measures $D_{\xi}(\tau(v))$ with $\tau \in \mathcal{T}$. 
Theorem 3.8. Let $v: \Omega \rightarrow \mathbb{R}$ be $\mathcal{L}^{n}$-measurable and let $\xi \in \mathbb{R}^{n} \backslash\{0\}$. Assume that conditions (a) and (b) of Theorem 3.5 are satisfied, and let $\mu^{\xi}$ be the measure introduced in Definition 3.7. Then for every open set $U \subset \Omega$ we have

$$
\mu^{\xi}(U):=\sup _{k} \sup \sum_{i=1}^{k}\left|D_{\xi}\left(\tau_{i}(v)\right)\right|\left(U_{i}\right)
$$

where the second supremum is over all families $\tau_{1}, \ldots, \tau_{k}$ of elements of $\mathcal{T}$ and all families $U_{1}, \ldots, U_{k}$ of pairwise disjoint open subsets of $U$. In other words, $\mu^{\xi}$ coincides with the smallest measure $\lambda$ such that (3.13) holds for every $\tau \in \mathcal{T}$.

Proof. In the proof of the implication (b) $\Rightarrow$ (a) in Theorem 3.5 we have already shown that $\left|D_{\xi}(\tau(v))\right|(B) \leq \mu^{\xi}(B)$ for every Borel set $B \subset \Omega$. This implies that the right-hand side of (3.25) is less than or equal to $\mu^{\xi}(U)$.

To prove the opposite inequality we fix an open set $U \subset \Omega$. By modifying $v$ on a set of Lebesgue measure zero, we may assume that $v$ is a Borel function on $\Omega$ and that $v_{y}^{\xi} \in \mathrm{BV}_{\text {loc }}\left(\Omega_{y}^{\xi}\right)$ for every $y \in \Pi^{\xi}$. Let $v_{+}^{\xi}$ and $v_{-}^{\xi}$ be the Borel functions defined by (3.16) and let $E:=\left\{x \in \Omega: v_{+}^{\xi}(x) \neq v_{-}^{\xi}(x)\right\}$ and $F:=\left\{x \in E:\left|v_{+}^{\xi}(x)-v_{-}^{\xi}(x)\right| \geq 1\right\}$. For every $y \in \Pi^{\xi}$ we have $\left(v_{+}^{\xi}\right)_{y}^{\xi}=\left(v_{-}^{\xi}\right)_{y}^{\xi}=v_{y}^{\xi} \mathcal{L}^{1}$-a.e. in $\Omega_{y}^{\xi}$ by Lebesgue's differentiation theorem. By elementary properties of BV functions in dimension one, for every $y \in \Pi^{\xi}$ this implies

$$
\begin{gathered}
\lim _{\rho \rightarrow 0+} \frac{1}{2 \rho} \int_{t-\rho}^{t+\rho}\left|v_{y}^{\xi}(s)-\left(v_{+}^{\xi}\right)_{y}^{\xi}(t)\right| d s=0, \\
\left(v_{y}^{\xi}\right)^{+}(t)=\left(v_{+}^{\xi}\right)_{y}^{\xi}(t) \quad \text { and } \quad\left(v_{y}^{\xi}\right)^{-}(t)=\left(v_{-}^{\xi}\right)_{y}^{\xi}(t)
\end{gathered}
$$

for every $t \in \Omega_{y}^{\xi} \backslash E_{y}^{\xi}$. This implies that $J_{v_{y}^{\xi}}=E_{y}^{\xi}$ and $J_{v_{y}^{\xi}}^{1}=F_{y}^{\xi}$ for every $y \in \Pi^{\xi}$.

For every $0<\varepsilon<1$ we can find three sequences of pairwise disjoint Borel sets $A_{i}$, $B_{i}, C_{i}$ and six sequences of real numbers $a_{i}^{1}, a_{i}^{2}, b_{i}^{1}, b_{i}^{2}, c_{i}^{1}, c_{i}^{2}$ such that

$$
\begin{gathered}
U \backslash E=\bigcup_{i} A_{i}, \quad U \cap E \backslash F=\bigcup_{i} B_{i}, \quad U \cap F=\bigcup_{i} C_{i} \\
a_{i}^{1}<v_{+}^{\xi}(x)<a_{i}^{2} \quad \text { for every } x \in A_{i}, \\
b_{i}^{1}<\min \left\{v_{+}^{\xi}(x), v_{-}^{\xi}(x)\right\}<\max \left\{v_{+}^{\xi}(x), v_{-}^{\xi}(x)\right\}<b_{i}^{2} \quad \text { for every } x \in B_{i}, \\
\min \left\{v_{+}^{\xi}(x), v_{-}^{\xi}(x)\right\}<c_{i}^{1}<c_{i}^{2}<\max \left\{v_{+}^{\xi}(x), v_{-}^{\xi}(x)\right\} \quad \text { for every } x \in C_{i}, \\
a_{i}^{1}<a_{i}^{2}<a_{i}^{1}+1, \quad b_{i}^{1}<b_{i}^{2}<b_{i}^{1}+1, \quad c_{i}^{1}+1<c_{i}^{2}+\varepsilon .
\end{gathered}
$$

By (3.28) we have

$$
\mu^{\xi}(U)=\sum_{i=1}^{\infty} \mu^{\xi}\left(A_{i}\right)+\sum_{i=1}^{\infty} \mu^{\xi}\left(B_{i}\right)+\sum_{i=1}^{\infty} \mu^{\xi}\left(C_{i}\right)
$$


Let us fix a constant $\alpha<\mu^{\xi}(U)$. Then there exists an integer $k>0$ such that

$$
\alpha<\sum_{i=1}^{k} \mu^{\xi}\left(A_{i}\right)+\sum_{i=1}^{k} \mu^{\xi}\left(B_{i}\right)+\sum_{i=1}^{k} \mu^{\xi}\left(C_{i}\right) .
$$

By standard approximation properties there exist pairwise disjoint compact sets $\hat{A}_{1}, \ldots$, $\hat{A}_{k}, \hat{B}_{1}, \ldots, \hat{B}_{k}, \hat{C}_{1}, \ldots, \hat{C}_{k}$, with $\hat{A}_{i} \subset A_{i}, \hat{B}_{i} \subset B_{i}$, and $\hat{C}_{i} \subset C_{i}$, such that

$$
\alpha<\sum_{i=1}^{k} \mu^{\xi}\left(\hat{A}_{i}\right)+\sum_{i=1}^{k} \mu^{\xi}\left(\hat{B}_{i}\right)+\sum_{i=1}^{k} \mu^{\xi}\left(\hat{C}_{i}\right)
$$

Let $\tilde{A}_{1}, \ldots, \tilde{A}_{k}, \tilde{B}_{1}, \ldots, \tilde{B}_{k}, \tilde{C}_{1}, \ldots, \tilde{C}_{k}$ be pairwise disjoint open subsets of $U$ with $\hat{A}_{i} \subset \tilde{A}_{i}, \hat{B}_{i} \subset \tilde{B}_{i}$, and $\hat{C}_{i} \subset \tilde{C}_{i}$.

By (3.32) for every $i$ there exists $\rho_{i} \in \mathcal{T}$ such that $\rho_{i}^{\prime}(s)=1$ for every $s \in\left[a_{i}^{1}, a_{i}^{2}\right]$. Since $\hat{A}_{i} \subset A_{i}$ and $\left(A_{i}\right)_{y}^{\xi} \cap J_{v_{y}^{\xi}}=\emptyset$, from (3.17) and (3.29) we obtain, using Vol'pert's chain rule in $\mathrm{BV}$ (see [6, Theorem 3.96]) and (3.26),

$$
\mu_{y}^{\xi}\left(\left(\hat{A}_{i}\right)_{y}^{\xi}\right)=\left|D v_{y}^{\xi}\right|\left(\left(\hat{A}_{i}\right)_{y}^{\xi}\right)=\int_{\left(\hat{A}_{i}\right)_{y}^{\xi}} \rho_{i}^{\prime}\left(\left(v_{+}^{\xi}\right)_{y}^{\xi}\right) d\left|D v_{y}^{\xi}\right|=\left|D\left(\rho_{i}\left(v_{y}^{\xi}\right)\right)\right|\left(\left(\hat{A}_{i}\right)_{y}^{\xi}\right)
$$

for every $i$ and every $y \in \Pi^{\xi}$. Integrating over $\Pi^{\xi}$ and using Proposition 3.1 and (3.18) we obtain

$$
\mu^{\xi}\left(\hat{A}_{i}\right)=\left|D_{\xi}\left(\rho_{i}(v)\right)\right|\left(\hat{A}_{i}\right) \leq\left|D_{\xi}\left(\rho_{i}(v)\right)\right|\left(\tilde{A}_{i}\right) .
$$

By (3.32) for every $i$ there exists $\sigma_{i} \in \mathcal{T}$ such that $\left|\sigma_{i}\left(s_{2}\right)-\sigma_{i}\left(s_{1}\right)\right|=\left|s_{2}-s_{1}\right|$ for every $s_{1}, s_{2} \in\left[b_{i}^{1}, b_{i}^{2}\right]$. Since $\hat{B}_{i} \subset B_{i}$ and $\left(B_{i}\right)_{y}^{\xi} \subset J_{v_{y}^{\xi}} \backslash J_{v_{y}^{\xi}}^{1}$, from (3.17) and (3.30) we obtain, using Vol'pert's chain rule in BV and (3.27),

$$
\begin{aligned}
\mu_{y}^{\xi}\left(\left(\hat{B}_{i}\right)_{y}^{\xi}\right) & =\int_{\left(\hat{B}_{i}\right)_{y}^{\xi}}\left|\left(v_{y}^{\xi}\right)^{+}-\left(v_{y}^{\xi}\right)^{-}\right| d \mathcal{H}^{0}=\int_{\left(\hat{B}_{i}\right)_{y}^{\xi}}\left|\sigma_{i}\left(\left(v_{y}^{\xi}\right)^{+}\right)-\sigma_{i}\left(\left(v_{y}^{\xi}\right)^{-}\right)\right| d \mathcal{H}^{0} \\
& =\left|D\left(\sigma_{i}\left(v_{y}^{\xi}\right)\right)\right|\left(\left(\hat{B}_{i}\right)_{y}^{\xi}\right)
\end{aligned}
$$

for every $i$ and every $y \in \Pi^{\xi}$. Integrating over $\Pi^{\xi}$ and using Proposition 3.1 and (3.18) we obtain

$$
\mu^{\xi}\left(\hat{B}_{i}\right)=\left|D_{\xi}\left(\sigma_{i}(v)\right)\right|\left(\hat{B}_{i}\right) \leq\left|D_{\xi}\left(\sigma_{i}(v)\right)\right|\left(\tilde{B}_{i}\right) .
$$

By (3.32) for every $i$ there exists $\tau_{i} \in \mathcal{T}$ such that $\tau_{i}\left(s_{2}\right)-\tau_{i}\left(s_{1}\right) \geq 1-\varepsilon$ for every $s_{1}<c_{i}^{1}<c_{i}^{2}<s_{2}$. Since $\hat{C}_{i} \subset C_{i}$ and $\left(C_{i}\right)_{y}^{\xi} \subset J_{v_{y}^{\xi}}^{1}$, from (3.17) and (3.31) we obtain, using Vol'pert's chain rule in BV and (3.27),

$$
\begin{aligned}
(1-\varepsilon) \mu_{y}^{\xi}\left(\left(\hat{C}_{i}\right)_{y}^{\xi}\right) & =(1-\varepsilon) \mathcal{H}^{0}\left(\left(\hat{C}_{i}\right)_{y}^{\xi}\right) \\
& \leq \int_{\left(\hat{C}_{i}\right)_{y}^{\xi}}\left|\tau_{i}\left(\left(v_{y}^{\xi}\right)^{+}\right)-\tau_{i}\left(\left(v_{y}^{\xi}\right)^{-}\right)\right| d \mathcal{H}^{0}=\left|D\left(\tau_{i}\left(v_{y}^{\xi}\right)\right)\right|\left(\left(\hat{C}_{i}\right)_{y}^{\xi}\right)
\end{aligned}
$$


for every $i$ and every $y \in \Pi^{\xi}$. Integrating over $\Pi^{\xi}$ and using Proposition 3.1 and (3.18) we obtain

$$
(1-\varepsilon) \mu^{\xi}\left(\hat{C}_{i}\right)=\left|D_{\xi}\left(\tau_{i}(v)\right)\right|\left(\hat{C}_{i}\right) \leq\left|D_{\xi}\left(\tau_{i}(v)\right)\right|\left(\tilde{C}_{i}\right) .
$$

By (3.33) and (3.34)-(3.36) we obtain

$$
(1-\varepsilon) \alpha<\sum_{i=1}^{k}\left|D_{\xi}\left(\rho_{i}(v)\right)\right|\left(\tilde{A}_{i}\right)+\sum_{i=1}^{k}\left|D_{\xi}\left(\sigma_{i}(v)\right)\right|\left(\tilde{B}_{i}\right)+\sum_{i=1}^{k}\left|D_{\xi}\left(\tau_{i}(v)\right)\right|\left(\tilde{C}_{i}\right) .
$$

This concludes the proof of (3.28), since $\alpha<\mu^{\xi}(U)$ and $0<\varepsilon<1$ are arbitrary.

\section{Definition and first properties}

In this section we define the space $\operatorname{GBD}(\Omega)$ of generalised functions of bounded deformation and the space $\operatorname{GSBD}(\Omega)$ of generalised special functions of bounded deformation.

Definition 4.1. The space $\operatorname{GBD}(\Omega)$ of generalised functions of bounded deformation is the space of all $\mathcal{L}^{n}$-measurable functions $u: \Omega \rightarrow \mathbb{R}^{n}$ with the following property: there exists $\lambda \in \mathcal{M}_{b}^{+}(\Omega)$ such that the following equivalent (see Theorem 3.5) conditions hold for every $\xi \in \mathbb{S}^{n-1}$ :

(a) for every $\tau \in \mathcal{T}$ the partial derivative $D_{\xi}(\tau(u \cdot \xi))$ belongs to $\mathcal{M}_{b}(\Omega)$ and its total variation satisfies

$$
\left|D_{\xi}(\tau(u \cdot \xi))\right|(B) \leq \lambda(B) \quad \text { for every Borel set } B \subset \Omega ;
$$

(b) for $\mathcal{H}^{n-1}$-a.e. $y \in \Pi^{\xi}$ the function $\hat{u}_{y}^{\xi}:=u_{y}^{\xi} \cdot \xi$ belongs to $\mathrm{BV}_{\text {loc }}\left(\Omega_{y}^{\xi}\right)$ and

$$
\int_{\Pi^{\xi}}\left(\left|D \hat{u}_{y}^{\xi}\right|\left(B_{y}^{\xi} \backslash J_{\hat{u}_{y}^{\xi}}^{1}\right)+\mathcal{H}^{0}\left(B_{y}^{\xi} \cap J_{\hat{u}_{y}^{\xi}}^{1}\right)\right) d \mathcal{H}^{n-1}(y) \leq \lambda(B)
$$

for every Borel set $B \subset \Omega$.

Definition 4.2. The space $\operatorname{GSBD}(\Omega)$ of generalised special functions of bounded deformation is the set of all functions $u \in \operatorname{GBD}(\Omega)$ such that for every $\xi \in \mathbb{S}^{n-1}$ and for $\mathcal{H}^{n-1}$-a.e. $y \in \Pi^{\xi}$ the function $\hat{u}_{y}^{\xi}:=u_{y}^{\xi} \cdot \xi$ belongs to $\operatorname{SBV}_{\text {loc }}\left(\Omega_{y}^{\xi}\right)$ (see [6, Section 4.1] for the definition of this space).

Remark 4.3. Arguing as in the proof of Proposition 3.4 we can prove that condition (b) of Definition 4.1 is equivalent to

(b') for $\mathcal{H}^{n-1}$-a.e. $y \in \Pi^{\xi}$ the function $\hat{u}_{y}^{\xi}:=u_{y}^{\xi} \cdot \xi$ belongs to $\operatorname{GBV}\left(\Omega_{y}^{\xi}\right)$ and

$$
\int_{\Pi^{\xi}}\left(\left|D\left(\sigma_{a}\left(\hat{u}_{y}^{\xi}\right)\right)\right|\left(B_{y}^{\xi} \backslash J_{\sigma_{a}\left(\hat{u}_{y}^{\xi}\right)}^{1}\right)+\mathcal{H}^{0}\left(B_{y}^{\xi} \cap J_{\sigma_{a}\left(\hat{u}_{y}^{\xi}\right)}^{1}\right)\right) d \mathcal{H}^{n-1}(y) \leq \lambda(B)
$$

for every Borel set $B \subset \Omega$ and every $a>0$,

where $\sigma_{a}$ be the truncation function defined by $\sigma_{a}(t)=-a$ for $t \leq-a, \sigma_{a}(t)=t$ for $-a \leq t \leq a$, and $\sigma_{a}(t)=a$ for $t \geq a$. For the same reason, Definition 4.2 does not change if $\hat{u}_{y}^{\xi} \in \operatorname{SBV}_{\text {loc }}\left(\Omega_{y}^{\xi}\right)$ is replaced by $\hat{u}_{y}^{\xi} \in \operatorname{GSBV}\left(\Omega_{y}^{\xi}\right)$ (see [6, Section 4.5] for the definition of this space). 
Remark 4.4. When $n=1$ the space $\operatorname{GBD}(\Omega)$ reduces to $\left\{u \in \mathrm{BV}_{\text {loc }}(\Omega):|D u|(\Omega)<\infty\right\}$ and $\operatorname{GSBD}(\Omega)$ reduces to $\left\{u \in \operatorname{SBV}_{\text {loc }}(\Omega):|D u|(\Omega)<\infty\right\}$. In the case $n>1$, using the slicing theory for BV functions developed in [6, Section 3.11], we can prove that if $u \in[\operatorname{GBV}(\Omega)]^{n}$ (see [6, Definition 4.26]) and $u$ satisfies the natural estimate considered in $\left[6\right.$, Theorem 4.40], then $u \in \operatorname{GBD}(\Omega)$. A similar result holds for $[\mathrm{GSBV}(\Omega)]^{n}$ and $\operatorname{GSBD}(\Omega)$.

Remark 4.5. Let $u \in \mathrm{BD}(\Omega)$. By the structure theorem for BD functions (see [5, Theorem 4.5]) for every $\xi \in \mathbb{S}^{n-1}$ and for $\mathcal{H}^{n-1}$-a.e. $y \in \Pi^{\xi}$ we have $\hat{u}_{y}^{\xi} \in \operatorname{BV}\left(\Omega_{y}^{\xi}\right)$ and

$$
\int_{\Pi \xi}\left(\left|D \hat{u}_{y}^{\xi}\right|\left(B_{y}^{\xi} \backslash J_{\hat{u}_{y}^{\xi}}^{1}\right)+\mathcal{H}^{0}\left(B_{y}^{\xi} \cap J_{\hat{u}_{y}^{\xi}}^{1}\right)\right) d \mathcal{H}^{n-1}(y) \leq|E u|(B),
$$

where $E u$ is the matrix-valued Radon measure defined by $(E u)_{i j}:=\frac{1}{2}\left(D_{i} u_{j}+D_{j} u_{i}\right)$. It follows that $\operatorname{BD}(\Omega) \subset \operatorname{GBD}(\Omega)$. Using [5, Proposition 4.7] we can also prove that $\operatorname{SBD}(\Omega) \subset \operatorname{GSBD}(\Omega)$. These inclusions are strict, as shown in Example 12.3.

Remark 4.6. Let $\sigma: \mathbb{R} \rightarrow \mathbb{R}$ be the truncation function defined by $\sigma(s):=\min \{|s|, 1\}$. Since

$$
\left|D \hat{u}_{y}^{\xi}\right|\left(B \cap J_{\hat{u}_{y}^{\xi}}\right)=\int_{B \cap J_{\hat{u}_{y}^{\xi}}}\left|\left[\hat{u}_{y}^{\xi}\right]\right| d \mathcal{H}^{0}
$$

for every Borel set $B \subset \Omega_{y}^{\xi}$, inequality (4.2) is equivalent to

$$
\int_{\Pi^{\xi}}\left(\left|D \hat{u}_{y}^{\xi}\right|\left(B_{y}^{\xi} \backslash J_{\hat{u}_{y}^{\xi}}\right)+\int_{B_{y}^{\xi} \cap J_{\hat{u}}^{\xi}} \sigma\left(\left[\hat{u}_{y}^{\xi}\right]\right) d \mathcal{H}^{0}\right) d \mathcal{H}^{n-1}(y) \leq \lambda(B)
$$

for every Borel set $B \subset \Omega$. Using the fact that $\left|D \hat{u}_{y}^{\xi}\right|(\{t\})=0$ for every $t \in \Omega_{y}^{\xi} \backslash J_{\hat{u}_{y}^{\xi}}$, we can write the previous inequality as

$$
\int_{\Pi \xi}\left(\left|D \hat{u}_{y}^{\xi}\right|\left(B_{y}^{\xi} \backslash J_{\xi, y}\right)+\int_{B_{y}^{\xi} \cap J_{\xi, y}} \sigma\left(\left[\hat{u}_{y}^{\xi}\right]\right) d \mathcal{H}^{0}\right) d \mathcal{H}^{n-1}(y) \leq \lambda(B),
$$

where $J_{\xi, y}$ is an arbitrary countable set containing $J_{\hat{u}_{y}^{\xi}}$ and $\left[\hat{u}_{y}^{\xi}\right](t):=0$ for every $t \in$ $\Omega_{y}^{\xi} \backslash J_{\hat{u}_{y}^{\xi}}$.

Since $\sigma(s+t) \leq \sigma(s)+\sigma(t)$ and $\sigma(\rho s) \leq \max \{|\rho|, 1\} \sigma(s)$ for every $s, t, \rho \in \mathbb{R}$, we deduce from condition (b) of Definition 4.1 and from (4.3) that $\operatorname{GBD}(\Omega)$ and $\operatorname{GSBD}(\Omega)$ are vector subspaces of the vector space of all $\mathcal{L}^{n}$-measurable functions from $\Omega$ to $\mathbb{R}^{n}$.

Remark 4.7. For every $B \subset \Omega$, every $\rho \in \mathbb{R}$, and every $\xi \in \mathbb{R}^{n} \backslash\{0\}$ we have $\rho B_{y}^{\rho \xi}=B_{y}^{\xi}$. Moreover, for every $u: \Omega \rightarrow \mathbb{R}^{n}$ and every $t \in \Omega_{y}^{\rho \xi}$ we have $\hat{u}_{y}^{\rho \xi}(t)=\rho \hat{u}_{y}^{\xi}(\rho t)$. It follows that, if $u \in \operatorname{GBD}(\Omega)$ and $\xi \in \mathbb{R}^{n} \backslash\{0\}$, then $u_{y}^{\xi} \in \operatorname{BV}_{\text {loc }}\left(\Omega_{y}^{\xi}\right)$ for $\mathcal{H}^{n-1}$-a.e. $y \in \Pi^{\xi}$ and the left-hand side of (4.2) is finite. 
Definition 4.8. Let $u \in \operatorname{GBD}(\Omega)$, let $\xi \in \mathbb{S}^{n-1}$, and let $y \in \mathbb{R}^{n}$ with $\hat{u}_{y}^{\xi} \in \operatorname{BV}\left(\Omega_{y}^{\xi}\right)$, $\left|D \hat{u}_{y}^{\xi}\right|\left(\Omega_{y}^{\xi}\right)<\infty$, and $\mathcal{H}^{0}\left(J_{\hat{u}_{y}^{\xi}}^{1}\right)<\infty$. The measure $\hat{\mu}_{y}^{\xi} \in \mathcal{M}_{b}^{+}\left(\Omega_{y}^{\xi}\right)$ is defined by

$$
\hat{\mu}_{y}^{\xi}(B):=\left|D \hat{u}_{y}^{\xi}\right|\left(B \backslash J_{\hat{u}_{y}^{\xi}}^{1}\right)+\mathcal{H}^{0}\left(B \cap J_{\hat{u}_{y}^{\xi}}^{1}\right) \quad \text { for every Borel set } B \subset \Omega_{y}^{\xi} .
$$

Remark 4.9. By condition (b) of Definition 4.1 the measure $\hat{\mu}_{y}^{\xi}$ is defined for $\mathcal{H}^{n-1}$ a.e. $y \in \Pi^{\xi}$. More in general, an easy change of variables shows that $\hat{\mu}_{y}^{\xi}$ is defined for $\mathcal{H}^{n-1}$-a.e. $y \in \Pi^{\eta}$ for every $\eta \in \mathbb{S}^{n-1}$ with $\eta \cdot \xi \neq 0$.

Definition 4.10. Let $u \in \operatorname{GBD}(\Omega)$ and let $\xi \in \mathbb{S}^{n-1}$. The measure $\hat{\mu}^{\xi} \in \mathcal{M}_{b}^{+}(\Omega)$ is defined by

$$
\hat{\mu}^{\xi}(B):=\int_{\Pi^{\xi}} \hat{\mu}_{y}^{\xi}\left(B_{y}^{\xi}\right) d \mathcal{H}^{n-1}(y) \quad \text { for every Borel set } B \subset \Omega .
$$

We use the notation $\left(\hat{\mu}_{u}\right)_{y}^{\xi}$ and $\hat{\mu}_{u}^{\xi}$ when we want to underline the dependence on $u$.

Remark 4.11. If $\eta \in \mathbb{S}^{n-1}$ and $\eta \cdot \xi \neq 0$, an obvious change of variables shows that

$$
\hat{\mu}^{\xi}(B)=|\eta \cdot \xi| \int_{\Pi^{\eta}} \hat{\mu}_{y}^{\xi}\left(B_{y}^{\xi}\right) d \mathcal{H}^{n-1}(y) \quad \text { for every Borel set } B \subset \Omega \text {. }
$$

Remark 4.12. The measures $\hat{\mu}_{y}^{\xi}$ and $\hat{\mu}^{\xi}$ corresponding to $u \in \operatorname{GBD}(\Omega)$ coincide with the measures $\mu_{y}^{\xi}$ and $\mu^{\xi}$ introduced in Definition 3.7 for the scalar function $v:=u \cdot \xi$. By (4.2), (4.4), and (4.5) we have

$$
\hat{\mu}^{\xi}(B) \leq \lambda(B) \quad \text { for every Borel set } B \subset \Omega .
$$

Remark 4.13. Let $u \in \mathrm{BD}(\Omega)$. By [5, Theorem 4.5] and by the area formula (see, e.g., [6, Theorem 2.71]) for every $\xi \in \mathbb{S}^{n-1}$ and every Borel set $B \subset \Omega$ we have

$$
\hat{\mu}_{u}^{\xi}(B)=|E u \xi \cdot \xi|\left(B \backslash J_{u}\right)+\int_{B \cap J_{u}} \sigma([u] \cdot \xi)\left|v_{u} \cdot \xi\right| d \mathcal{H}^{n-1} \leq|E u \xi \cdot \xi|(B)
$$

where $\sigma$ is the function introduced in Remark 4.6.

Remark 4.14. Let $u \in \operatorname{GBD}(\Omega)$. For every $\tau \in \mathcal{T}$, every open set $U \subset \Omega$, and every $\varphi \in C_{c}^{1}(\Omega)$ the function

$$
\xi \mapsto \int_{U} \tau(u \cdot \xi) \nabla \varphi \cdot \xi d x
$$

is continuous on $\mathbb{S}^{n-1}$. Since

$$
\left|D_{\xi}(\tau(u \cdot \xi))\right|(U)=\sup _{\substack{\varphi \in C_{c}^{1}(\Omega) \\|\varphi| \leq 1}} \int_{U} \tau(u \cdot \xi) \nabla \varphi \cdot \xi d x,
$$

the function $\xi \mapsto\left|D_{\xi}(\tau(u \cdot \xi))\right|(U)$ is lower semicontinuous on $\mathbb{S}^{n-1}$. By Theorem 3.8 and Remark 4.12 it follows that $\xi \mapsto \hat{\mu}^{\xi}(U)$ is lower semicontinuous on $\mathbb{S}^{n-1}$. 
Remark 4.15. By standard properties of bounded measures, it is enough to check the inequalities in (4.1) and (4.2) when $B \in \mathcal{B}$, where $\mathcal{B}$ is a base for the topology of $\Omega$ and $\mathcal{B}$ is stable under finite unions and intersections. By the lower semicontinuity of $\xi \mapsto\left|D_{\xi}(\tau(u \cdot \xi))\right|(U)$ when $U$ is open (Remark 4.14), it is enough to check (4.1) for every $\xi$ in a dense subset $\Xi$ of $\mathbb{S}^{n-1}$. Since conditions (a) and (b) of Definition 4.1 are equivalent for every $\xi$ by Theorem 3.5 , it is enough that one of them is satisfied for every $B \in \mathcal{B}$ and every $\xi \in \Xi$.

Definition 4.16. For every $u \in \operatorname{GBD}(\Omega)$ let $\hat{\mu}_{u} \in \mathcal{M}_{b}^{+}(\Omega)$ be the measure defined by setting, for every Borel set $B \subset \Omega$,

$$
\hat{\mu}_{u}(B):=\sup _{k} \sup \sum_{i=1}^{k} \hat{\mu}_{u}^{\xi_{i}}\left(B_{i}\right),
$$

where the second supremum is over all families $\xi_{1}, \ldots, \xi_{k}$ of elements of $\mathbb{S}^{n-1}$ and over all families $B_{1}, \ldots, B_{k}$ of pairwise disjoint Borel subsets of $B$.

By (4.4) and (4.5) for every $u \in \operatorname{GBD}(\Omega)$ the measure $\hat{\mu}_{u}$ is the smallest measure $\lambda$ that satisfies condition (b) of Definition 4.1.

Proposition 4.17. Let $u \in \operatorname{GBD}(\Omega)$ and let $\lambda \in \mathcal{M}_{b}^{+}(\Omega)$ be the measure considered in Definition 4.1. Then for every Borel set $B \subset \Omega$ we have

$$
\hat{\mu}_{u}(B) \leq \lambda(B) \text {. }
$$

Moreover, if $\mathcal{H}^{n-1}\left(\pi^{\xi}(B)\right)=0$ for $\mathcal{H}^{n-1}$-a.e. $\xi \in \mathbb{S}^{n-1}$, where $\pi^{\xi}$ is the orthogonal projection onto $\Pi^{\xi}$, then $\hat{\mu}_{u}(B)=0$.

Proof. Inequality (4.9) follows from (4.6). To prove the second statement, we fix a Borel set $B_{0} \subset \Omega$. We consider the set $S_{0}:=\left\{\xi \in \mathbb{S}^{n-1}: \mathcal{H}^{n-1}\left(\pi^{\xi}\left(B_{0}\right)\right)=0\right\}$ and we assume that $\mathcal{H}^{n-1}\left(\mathbb{S}^{n-1} \backslash S_{0}\right)=0$. Let $\tilde{\mu}_{u}$ be the measure defined as in (4.8), with the constraint that $\xi_{1}, \ldots, \xi_{k}$ are now elements of $S_{0}$. By (4.6) we have

$$
\tilde{\mu}_{u}(B) \leq \lambda(B) \quad \text { for every Borel set } B \subset \Omega \text {. }
$$

Let $\tilde{\lambda}$ be the absolutely continuous part of $\lambda$ with respect to $\tilde{\mu}_{u}$. From (4.10) we deduce that $\tilde{\mu}_{u}(B) \leq \tilde{\lambda}(B)$ for every Borel set $B \subset \Omega$. Therefore the definition of $\tilde{\mu}_{u}$ gives $\mu_{u}^{\xi}(U) \leq \tilde{\lambda}(U)$ for every $\xi \in S_{0}$ and every open set $U \subset \Omega$. Since $\xi \mapsto \hat{\mu}_{u}^{\xi}(U)$ is lower semicontinuous on $\mathbb{S}^{n-1}$ by Remark 4.14 and $S_{0}$ is dense in $\mathbb{S}^{n-1}$, we conclude that $\mu_{u}^{\xi}(U) \leq \tilde{\lambda}(U)$ for every $\xi \in \mathbb{S}^{n-1}$ and every open set $U \subset \Omega$. It follows that $\mu_{u}^{\xi}(B) \leq \tilde{\lambda}(B)$ for every $\xi \in \mathbb{S}^{n-1}$ and every Borel set $B \subset \Omega$, which implies

$$
\hat{\mu}_{u}(B) \leq \tilde{\lambda}(B) \quad \text { for every Borel set } B \subset \Omega \text {. }
$$

Since by (4.5),

$$
\hat{\mu}^{\xi}(B):=\int_{\pi^{\xi}(B)} \hat{\mu}_{y}^{\xi}\left(B_{y}^{\xi}\right) d \mathcal{H}^{n-1}(y)
$$


we have $\hat{\mu}^{\xi}\left(B_{0}\right)=0$ for every $\xi \in S_{0}$. It follows that $\tilde{\mu}_{u}\left(B_{0}\right)=0$. As $\tilde{\lambda}$ is absolutely continuous with respect to $\tilde{\mu}_{u}$, we have also $\tilde{\lambda}\left(B_{0}\right)=0$. By (4.11) this gives $\hat{\mu}_{u}\left(B_{0}\right)=0$, which concludes the proof.

In the proof of the rectifiability of $J_{u}$ we need the following semicontinuity result.

Lemma 4.18. Let $u_{k}$ be a sequence in $\operatorname{GBD}(\Omega)$ converging in $\mathcal{L}^{n}$-measure to a function $u \in \operatorname{GBD}(\Omega)$. Then

$$
\hat{\mu}_{u}^{\xi}(U) \leq \liminf _{k \rightarrow \infty} \hat{\mu}_{u_{k}}^{\xi}(U)
$$

for every $\xi \in \mathbb{S}^{n-1}$ and every open set $U \subset \Omega$.

Proof. For every $\varphi \in C_{c}^{1}(\Omega)$ the function

$$
u \mapsto \int_{U} \tau(u \cdot \xi) \nabla \varphi \cdot \xi d x
$$

is lower semicontinuous with respect to convergence in $\mathcal{L}^{n}$-measure. By (4.7) the function $u \mapsto\left|D_{\xi}(\tau(u \cdot \xi))\right|(U)$ is lower semicontinuous. The conclusion follows now from Theorem 3.8 and Remark 4.12.

The following theorem concerns $k$-dimensional slices. For every linear subspace $V$ of $\mathbb{R}^{n}$ of dimension $k>0$ and for every bounded open set $\Omega_{V}$ in the relative topology of $V$, the space $\operatorname{GBD}\left(\Omega_{V}\right)$ is defined as in Definition 4.1, with $\Omega$ replaced by $\Omega_{V}, \mathbb{R}^{n}$ replaced by $V, \mathbb{S}^{n-1}$ replaced by $\mathbb{S}_{V}^{k-1}:=\mathbb{S}^{n-1} \cap V, \Pi^{\xi}$ replaced by $\Pi_{V}^{\xi}:=\Pi^{\xi} \cap V, \mathcal{L}^{n}$ replaced by $\mathcal{H}^{k}\left\llcorner V\right.$, and $\mathcal{H}^{n-1}$ replaced by $\mathcal{H}^{k-1}$.

Theorem 4.19. Let $V$ be a linear subspace of $\mathbb{R}^{n}$ of dimension $k>0$, let $V^{\perp}$ be its orthogonal subspace, and let $\pi_{V}$ be the orthogonal projection from $\mathbb{R}^{n}$ onto $V$. Given a function $u \in \operatorname{GBD}(\Omega)$, for every $y \in V^{\perp}$ let $\Omega_{y}:=\{z \in V: y+z \in \Omega\}$ and let $u_{y}: \Omega_{y} \rightarrow V$ be the function defined by $u_{y}(z):=\pi_{V}(u(y+z))$. Then $u_{y} \in \operatorname{GBD}\left(\Omega_{y}\right)$ for $\mathcal{H}^{n-k}$-a.e. $y \in V^{\perp}$.

Proof. By Fubini's theorem the function $u_{y}: \Omega_{y} \rightarrow V$ is $\mathcal{H}^{k}$-measurable on $\Omega_{y}$ for $\mathcal{H}^{n-k}$-a.e. $y \in V^{\perp}$. Let us prove that for $\mathcal{H}^{n-k}$-a.e. $y \in V^{\perp}$ there exists $\hat{\lambda}_{y} \in \mathcal{M}_{b}^{+}\left(\Omega_{y}\right)$ such that $u_{y}$ satisfies condition (b) of Definition 4.1 on $\Omega_{y} \subset V$ for every $\xi \in V$.

We begin by observing that, if $\xi \in V$, then the hyperplane $\Pi^{\xi}$ is the sum of the orthogonal subspaces $V^{\perp}$ and $\Pi_{V}^{\xi}:=\Pi^{\xi} \cap V$, of dimension $n-k$ and $k-1$, respectively. Since $\xi \in V$, we have $u \cdot \xi=\pi_{V}(u) \cdot \xi$. This implies that $\hat{u}_{y+z}^{\xi}=\widehat{\left(u_{y}\right)} z_{z}^{\xi}$ on $\Omega_{y+z}^{\xi}=\left(\Omega_{y}\right)_{z}^{\xi}$ for every $y \in V^{\perp}$ and every $z \in \Pi_{V}^{\xi}$.

For every Borel set $B \subset \Omega$ and every $y \in V^{\perp}$ we define $B_{y}:=\{z \in V: y+z \in B\}$, so that $B_{y+z}^{\xi}=\left(B_{y}\right)_{z}^{\xi}$ for every $z \in V$. Let $\omega=\pi_{V}(\lambda)$, let $N$ be a Borel subset of $V^{\perp}$, with $\mathcal{H}^{n-k}(N)=0$, such that the singular part of $\omega$ with respect to $\mathcal{H}^{n-k}\left\llcorner V^{\perp}\right.$ is concentrated on $N$, and let $g: V^{\perp} \rightarrow \mathbb{R}^{+}$be the density of the absolutely continuous part of $\omega$ with respect to $\mathcal{H}^{n-k}\left\llcorner V^{\perp}\right.$. By the disintegration theorem (see, e.g., [6, Theorem 2.28]) there 
exists a Borel measurable family $\left(\lambda_{y}\right)_{y \in V^{\perp}}$ of Radon measures, with $\lambda_{y} \in \mathcal{M}_{b}^{+}\left(\Omega_{y}\right)$, such that

$$
\lambda(B)=\int_{V^{\perp}} \lambda_{y}\left(B_{y}\right) d \omega(y) \quad \text { for every Borel set } B \subset \Omega .
$$

Let us fix a countable dense subset $\Xi$ of $\mathbb{S}_{V}^{k-1}:=\mathbb{S}^{n-1} \cap V$. By condition (b) of Definition 4.1 and by Fubini's theorem for $\mathcal{H}^{n-k}$-a.e. $y \in V^{\perp}$ the functions $\hat{u}_{y+z}^{\xi}=\left(\hat{u}_{y}\right)_{z}^{\xi}$ belong to $\mathrm{BV}_{\text {loc }}\left(\Omega_{y+z}^{\xi}\right)=\mathrm{BV}_{\text {loc }}\left(\left(\Omega_{y}\right)_{z}^{\xi}\right)$ for every $\xi \in \Xi$ and for $\mathcal{H}^{k-1}$-a.e. $z \in \Pi_{V}^{\xi}$.

Let $\mathcal{B}$ be a countable base for the topology of $V$ such that $U_{1} \cap U_{2} \in \mathcal{B}$ for every $U_{1}, U_{2} \in \mathcal{B}$. By Remark 4.15 to conclude the proof it is enough to show that for $\mathcal{H}^{n-k_{-}}$ a.e. $y \in V^{\perp}$ the function $u_{y}$ satisfies the analogue of (4.2) in $V$, with $\lambda$ replaced by $\hat{\lambda}_{y}:=g(y) \lambda_{y}$.

Given a Borel set $A \subset V^{\perp}$ and a open set $U \in \mathcal{B}$, we consider the Borel set $B \subset \Omega$ defined by $B:=\left\{y+z: y \in A, z \in U \cap \Omega_{y}\right\}$. Let $\tilde{A}:=A \backslash N$ and $\tilde{B}:=\{y+z: y \in \tilde{A}$, $z \in U \cap \Omega_{y}$ \}. By Fubini's theorem and by (4.4)-(4.6) and (4.13) we have

$$
\begin{aligned}
\int_{A}\left(\int _ { \Pi _ { V } ^ { \xi } } \hat { \mu } _ { y + z } ^ { \xi } \left(U_{z}^{\xi} \cap\right.\right. & \left.\left.\left(\Omega_{y}\right)_{z}^{\xi}\right) d \mathcal{H}^{k-1}(z)\right) d \mathcal{H}^{n-k}(y) \\
& =\int_{\tilde{A}}\left(\int_{\Pi_{V}^{\xi}} \hat{\mu}_{y+z}^{\xi}\left(U_{z}^{\xi} \cap\left(\Omega_{y}\right)_{z}^{\xi}\right) d \mathcal{H}^{k-1}(z)\right) d \mathcal{H}^{n-k}(y) \\
& =\int_{\Pi^{\xi}} \hat{\mu}_{x}^{\xi}\left(\tilde{B}_{x}^{\xi}\right) d \mathcal{H}^{n-1}(x)=\hat{\mu}^{\xi}(\tilde{B}) \leq \lambda(\tilde{B}) \\
& =\int_{\tilde{A}} \lambda_{y}\left(U \cap \Omega_{y}\right) d \omega(y)=\int_{A} \lambda_{y}\left(U \cap \Omega_{y}\right) g(y) \mathcal{H}^{n-k}(y) .
\end{aligned}
$$

Since this inequality holds for every Borel set $A \subset V^{\perp}$ we conclude that for every $U \in \mathcal{B}$ we have

$$
\int_{\Pi_{V}^{\xi}} \hat{\mu}_{y+z}^{\xi}\left(U_{z}^{\xi} \cap\left(\Omega_{y}\right)_{z}^{\xi}\right) d \mathcal{H}^{k-1}(z) \leq g(y) \lambda_{y}\left(U \cap \Omega_{y}\right)
$$

for $\mathcal{H}^{n-k}$-a.e. $y \in V^{\perp}$. Since $\mathcal{B}$ is countable, we conclude that for $\mathcal{H}^{n-k}$-a.e. $y \in V^{\perp}$ inequality (4.14) holds for every $U \in \mathcal{B}$. This shows that for $\mathcal{H}^{n-k}$-a.e. $y \in V^{\perp}$ the function $u_{y}$ satisfies condition (b) of Definition 4.1 on $V$ for every $\xi \in \Xi$ and every $B=U \in \mathcal{B}$, hence $u_{y} \in \operatorname{GBD}\left(\Omega_{V}\right)$ by Remark 4.15.

\section{Traces on regular submanifolds and on the boundary}

The following theorem summarizes the known results on the traces of functions $v \in$ $L^{1}(\Omega)$ satisfying $D_{\xi} v \in \mathcal{M}_{b}(\Omega)$ for some vector $\xi \in \mathbb{S}^{n-1}$.

Theorem 5.1. Let $U$ and $V$ be open subsets of $\mathbb{R}^{n}$ of the form

$$
U:=\{y+t \xi: y \in B, a<t<\psi(y)\} \text { and } V:=\{y+t \xi: y \in B, a<t<b\} \text {, }
$$


where $\xi \in \mathbb{S}^{n-1}, B$ is a relatively open ball in $\Pi^{\xi}, a, b \in \mathbb{R}$, with $a<b$, and $\psi: \bar{B} \rightarrow$ $(a, b)$ is Lipschitz continuous. Let $v \in L^{1}(\Omega)$ with $D_{\xi} v \in \mathcal{M}_{b}(\Omega)$, let

$$
M:=\{y+\psi(y) \xi: y \in B\},
$$

and let $v$ be the outer unit normal to $M$. Then there exists a function $v_{M} \in L_{\mathcal{H}^{n-1}}^{1}(M)$ such that

$$
\int_{U} v D_{\xi} \varphi d x+\int_{U} \varphi d\left(D_{\xi} v\right)=\int_{M} \varphi v_{M} \xi \cdot v d \mathcal{H}^{n-1}
$$

for every $\varphi \in C_{c}^{1}(V)$. Moreover

$$
\lim _{\rho \rightarrow 0+} \frac{1}{\rho^{n}} \int_{B_{\rho}(x) \cap U}\left|v(z)-v_{M}(x)\right| d z=0
$$

for $\mathcal{H}^{n-1}$-a.e. $x \in M$. Finally, for $\mathcal{H}^{n-1}$-a.e. $y \in \Pi^{\xi}$ we have

$$
v_{M}(y+\psi(y) \xi)=\underset{t \rightarrow \psi(y)-}{\operatorname{ap} \lim } v_{y}^{\xi}(t) .
$$

Proof. The existence of $v_{M} \in L_{\mathcal{H}^{n-1}}^{1}(M)$ satisfying (5.3) follows from [31, Lemma 1.1]. The proof of (5.4) can be obtained by slight modifications of the arguments of [18, Theorem 5.3.2], where the use of the coarea formula can be avoided. Equality (5.5) can be easily deduced from [31, formula (1.17)].

We are now in a position to prove the main result about traces of functions $u \in \operatorname{GBD}(\Omega)$ on a regular submanifold.

Theorem 5.2. Let $u \in \operatorname{GBD}(\Omega)$ and let $M \subset \Omega$ be a $C^{1}$ submanifold of dimension $n-1$ with unit normal $v$. Then for $\mathcal{H}^{n-1}$-a.e. $x \in M$ there exist $u_{M}^{+}(x), u_{M}^{-}(x) \in \mathbb{R}^{n}$ such that

$$
\underset{\substack{ \pm(y-x) \cdot v(x)>0 \\ y \rightarrow x}}{\operatorname{ap} \lim } u(y)=u_{M}^{ \pm}(x) .
$$

Moreover for every $\xi \in \mathbb{S}^{n-1}$ and for $\mathcal{H}^{n-1}$-a.e. $y \in \Pi^{\xi}$ we have

$$
u_{M}^{ \pm}(y+t \xi) \cdot \xi=\underset{\substack{\xi \\ \sigma_{y}^{\xi}(t)(s-t)>0 \\ s \rightarrow t}}{\operatorname{ap} \lim } \hat{u}_{y}^{\xi}(s) \quad \text { for every } t \in M_{y}^{\xi}
$$

where $\sigma: M \rightarrow\{-1,1\}$ is defined by $\sigma(x):=\operatorname{sign}(\xi \cdot v(x))$. Finally, the functions $u_{M}^{ \pm}: M \rightarrow \mathbb{R}^{n}$ are $\mathcal{H}^{n-1}$-measurable.

Proof. It is enough to prove (5.6) in a neighbourhood of each point. For every $x_{0} \in M$ there exist an open neighbourhood $A$ of $x_{0}$, a vector $\xi_{0} \in \mathbb{S}^{n-1}$, and a constant $0<\varepsilon<1$ such that for every $\xi \in \mathbb{S}^{n-1}$ with $\left|\xi-\xi_{0}\right|<\varepsilon$ we can represent $M \cap A$ as a Lipschitz graph in the direction determined by $\xi$ :

$$
M \cap A \subset\{y+\psi(y) \xi: y \in B\} \subset M,
$$


where $\psi, B, a$, and $b$ are as in Theorem 5.1. We may also assume that the set $V$ defined by (5.1) is contained in $\Omega$ and that $v(x) \cdot \xi>0$ for every $x \in M \cap V$. We set

$$
A^{-}:=A \cap U \quad \text { and } \quad A^{+}:=A \backslash\left(M \cup A^{-}\right),
$$

where $U$ is defined in (5.1).

Given $\tau \in \mathcal{T}$ with $\tau^{\prime}(t)>0$ for every $t \in \mathbb{R}$, we define $v:=\tau(u \cdot \xi)$. By condition (a) of Definition 4.1 and by Theorem 5.1 for $\mathcal{H}^{n-1}$-a.e. $x \in M \cap A$ there exist two real numbers $v_{M}^{+}(x)$ and $v_{M}^{-}(x)$ such that (5.4) holds with $v_{M}(x)$ replaced by $v_{M}^{ \pm}(x)$ and $U$ replaced by $A^{ \pm}$. This implies that

$$
\operatorname{ap}_{\substack{ \pm(y-x) \cdot v(x)>0 \\ y \rightarrow x}} \tau(u(y) \cdot \xi)=v_{M}^{ \pm}(x) .
$$

Moreover, by Theorem 5.1 the functions $v_{M}^{ \pm}: M \cap A \rightarrow \mathbb{R}$ are $\mathcal{H}^{n-1}$-measurable.

By (5.5) for $\mathcal{H}^{n-1}$-a.e. $y \in \Pi^{\xi}$ and for every $t \in M_{y}^{\xi} \cap A_{y}^{\xi}$ we have

$$
v_{M}^{ \pm}(y+t \xi)=\underset{s \rightarrow t \pm}{\operatorname{ap} \lim } v_{y}^{\xi}(s)=\tau\left(\underset{s \rightarrow t \pm}{\operatorname{ap} \lim } \hat{u}_{y}^{\xi}(s)\right),
$$

where the existence of the approximate limit of $\hat{u}_{y}^{\xi}$ follows from the fact that $\hat{u}_{y}^{\xi} \in$ $\mathrm{BV}_{\text {loc }}\left(\Omega_{y}^{\xi}\right)$ by condition (b) of Definition 4.1. By (5.9) we have $v_{M}^{ \pm}(x) \in \tau(\mathbb{R})$ for $\mathcal{H}^{n-1}$ a.e. $x \in M \cap A$. By inverting the function $\tau$ we deduce from (5.8) that for $\mathcal{H}^{n-1}$-a.e. $x \in M \cap A$ there exist two real numbers $u_{\xi, M}^{+}(x)$ and $u_{\xi, M}^{-}(x)$ such that

$$
\operatorname{ap}_{\substack{ \pm(y-x) \cdot v(x)>0 \\ y \rightarrow x}} u(y) \cdot \xi=u_{\xi, M}^{ \pm}(x) .
$$

Moreover, the functions $u_{\xi, M}^{ \pm}: M \cap A \rightarrow \mathbb{R}$ are $\mathcal{H}^{n-1}$-measurable. Since there exists a basis of $\mathbb{R}^{n}$ composed of vectors $\xi \in \mathbb{S}^{n-1}$ with $\left|\xi-\xi_{0}\right|<\varepsilon$, equality (5.10) implies that for $\mathcal{H}^{n-1}$-a.e. $x \in M \cap A$ there exist two vectors $u_{M}^{+}(x), u_{M}^{-}(x) \in \mathbb{R}^{n}$ satisfying (5.6) and such that the functions $u_{M}^{ \pm}: M \cap A \rightarrow \mathbb{R}^{n}$ are $\mathcal{H}^{n-1}$-measurable.

Let us prove (5.7) for an arbitrary $\xi \in \mathbb{S}^{n-1}$. Since $\mathcal{H}^{n-1}\left(\pi^{\xi}(\{x \in M: \xi \cdot \nu(x)=0\})\right)$ $=0$ by the area formula (see, e.g., [6, Theorem 2.91]), by localization we may assume that $M$ can be represented as in (5.2) and that $\xi \cdot v(x)>0$ for every $x \in M$. Let $\tau$ be as in the first part of the proof and let $U^{ \pm}:=\{y+t \xi: y \in B, a<t<b, \pm(t-\psi(y))>0\}$. By (5.6) we have

$$
\underset{\substack{y \in U^{ \pm} \\ y \rightarrow x}}{\operatorname{ap} \lim } \tau(u(y) \cdot \xi)=\tau\left(u_{M}^{ \pm}(x) \cdot \xi\right)
$$

for $\mathcal{H}^{n-1}$-a.e. $x \in M$. Since $\tau(u \cdot \xi)$ is bounded, this implies that

$$
\lim _{\rho \rightarrow 0+} \frac{1}{\rho^{n}} \int_{B_{\rho}(x) \cap U^{ \pm}}\left|\tau(u(y) \cdot \xi)-\tau\left(u_{M}^{ \pm}(x) \cdot \xi\right)\right| d y=0 .
$$


By Theorem 5.1, applied to $v:=\tau(u \cdot \xi)$, for $\mathcal{H}^{n-1}$-a.e. $y \in \Pi^{\xi}$ we have

$$
\tau\left(u_{M}^{ \pm}(y+t \xi) \cdot \xi\right)=\underset{s \rightarrow t \pm}{\operatorname{ap} \lim } \tau\left(\hat{u}_{y}^{\xi}(s)\right) \quad \text { for every } t \in M_{y}^{\xi}
$$

By inverting the function $\tau$ we conclude that (5.7) holds for $\mathcal{H}^{n-1}$-a.e. $x \in M$.

Definition 5.3. Let $u \in \operatorname{GBD}(\Omega)$ and let $M \subset \Omega$ be a $C^{1}$ submanifold of dimension $n-1$ with normal $v$. The $\mathbb{R}^{n}$-valued $\mathcal{H}^{n-1}$-measurable functions $u_{M}^{+}$and $u_{M}^{-}$, defined $\mathcal{H}^{n-1}$-a.e. on $M$ and satisfying (5.6), are called the traces of $u$ on the two sides of $M$.

Remark 5.4. Let $u \in \operatorname{GBD}(\Omega)$ and let $M \subset \Omega$ be a $C^{1}$ manifold of dimension $n-1$ with normal $v$. By (5.6) we have $\left\{x \in M: u_{M}^{+}(x) \neq u_{M}^{-}(x)\right\} \subset J_{u} \cap M$ and

$$
\mathcal{H}^{n-1}\left(J_{u} \cap M \backslash\left\{x \in M: u_{M}^{+}(x) \neq u_{M}^{-}(x)\right\}\right)=0 .
$$

Moreover

$$
\left(u^{+}(x), u^{-}(x), v_{u}(x)\right)=\left(u_{M}^{+}(x), u_{M}^{-}(x), v(x)\right) \quad \text { or } \quad=\left(u_{M}^{-}(x), u_{M}^{+}(x),-v(x)\right)
$$

for $\mathcal{H}^{n-1}$-a.e. $x \in J_{u} \cap M$.

When $\Omega$ has a Lipschitz boundary we can also consider traces on the boundary.

Theorem 5.5. Assume that $\Omega$ has a Lipschitz boundary and let $v$ be the outward unit normal. Then for every $u \in \operatorname{GBD}(\Omega)$ and for $\mathcal{H}^{n-1}$-a.e. $x \in \partial \Omega$ there exist $u_{\partial \Omega}(x) \in \mathbb{R}^{n}$ such that

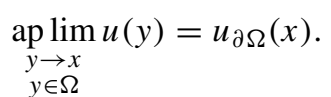

Moreover for every $\xi \in \mathbb{S}^{n-1}$ and for $\mathcal{H}^{n-1}$-a.e. $y \in \Pi^{\xi}$ we have

$$
u_{\partial \Omega}(y+t \xi) \cdot \xi=\operatorname{ap}_{\substack{\sigma_{y}^{\xi}(t)(s-t)>0 \\ s \rightarrow t}} \hat{u}_{y}^{\xi}(s) \quad \text { for every } t \in(\partial \Omega)_{y}^{\xi},
$$

where $\sigma: \partial \Omega \rightarrow\{-1,1\}$ is given by $\sigma(x):=\operatorname{sign}(\xi \cdot v(x))$. Finally, the function $u_{\partial \Omega}: \partial \Omega \rightarrow \mathbb{R}^{n}$ is $\mathcal{H}^{n-1}$-measurable.

Proof. The proof is similar to the proof of Theorem 5.2, and therefore is omitted.

Definition 5.6. Assume that $\Omega$ has a Lipschitz boundary. For every $u \in \operatorname{GBD}(\Omega)$ the $\mathbb{R}^{n}$-valued $\mathcal{H}^{n-1}$-measurable function $u_{\partial \Omega}$, defined $\mathcal{H}^{n-1}$-a.e. on $\partial \Omega$ and satisfying (5.11), is called the trace of $u$ on $\partial \Omega$.

\section{Rectifiability of the jump set}

In this section we prove that for every $u \in \operatorname{GBD}(\Omega)$ the jump set $J_{u}$ introduced in Definition 2.4 is countably $\left(\mathcal{H}^{n-1}, n-1\right)$-rectifiable according to [19, Section 3.2.14]. We recall that, by [19, Theorem 3.2.29], a set $E \subset \mathbb{R}^{n}$ is countably $\left(\mathcal{H}^{n-1}, n-1\right)$-rectifiable if and only if $\mathcal{H}^{n-1}$-almost all of $E$ is contained in the union of a countable family of $(n-1)$-dimensional submanifolds of $\mathbb{R}^{n}$ of class $C^{1}$. 
To prove the rectifiability of $J_{u}$, for every $u \in \operatorname{GBD}(\Omega)$ we consider the set

$$
\Theta_{u}:=\left\{x \in \Omega: \limsup _{\rho \rightarrow 0+} \frac{\hat{\mu}_{u}\left(B_{\rho}(x)\right)}{\rho^{n-1}}>0\right\},
$$

where $\hat{\mu}_{u}$ is the measure introduced in Definition 4.16.

Proposition 6.1. Let $u \in \operatorname{GBD}(\Omega)$. Then $\Theta_{u}$ is a countably $\left(\mathcal{H}^{n-1}, n-1\right)$-rectifiable Borel set.

Proof. The proof is a variant of the proof of [23, Part II, Theorem 4.18]. By Fatou's lemma for every $\rho>0$ the function $x \mapsto \hat{\mu}_{u}\left(B_{\rho}(x) \cap \Omega\right)$ is lower semicontinuous on $\Omega$. Since the limsup can be computed by considering only rational numbers $\rho>0$, we deduce that $\Theta_{u}$ is a Borel set.

To prove the rectifiability, for every $\varepsilon>0$ we consider the Borel set

$$
\Theta_{u}^{\varepsilon}:=\left\{x \in \Omega: \limsup _{\rho \rightarrow 0+} \frac{\hat{\mu}_{u}\left(B_{\rho}(x)\right)}{\rho^{n-1}}>\varepsilon\right\} .
$$

It is enough to show that $\Theta_{u}^{\varepsilon}$ is countably $\left(\mathcal{H}^{n-1}, n-1\right)$-rectifiable. By [19, Theorem 2.10.19] we have

$$
\varepsilon \mathcal{H}^{n-1}(B) \leq \omega_{n} \hat{\mu}_{u}(B)
$$

for every Borel set $B \subset \Theta_{u}^{\varepsilon}$. In particular $\mathcal{H}^{n-1}\left(\Theta_{u}^{\varepsilon}\right)<\infty$ and we can apply Federer's structure theorem [19, Theorems 3.3.13 and 2.10.15] to obtain a countably $\left(\mathcal{H}^{n-1}, n-1\right)$ rectifiable Borel set $R \subset \Theta_{u}^{\varepsilon}$ such that

$$
\mathcal{H}^{n-1}\left(\pi^{\xi}\left(\Theta_{u}^{\varepsilon} \backslash R\right)\right)=0 \quad \text { for } \mathcal{H}^{n-1} \text {-a.e. } \xi \in \mathbb{S}^{n-1} .
$$

By Proposition 4.17 we have $\hat{\mu}_{u}\left(\Theta_{u}^{\varepsilon} \backslash R\right)=0$. Choosing $B=\Theta_{u}^{\varepsilon} \backslash R$ in (6.3) we obtain $\mathcal{H}^{n-1}\left(\Theta_{u}^{\varepsilon} \backslash R\right)=0$. This proves that $\Theta_{u}^{\varepsilon}$ is countably $\left(\mathcal{H}^{n-1}, n-1\right)$-rectifiable and concludes the proof of the proposition.

We are now in a position to prove that the jump set of a function of $\operatorname{GBD}(\Omega)$ is countably $\left(\mathcal{H}^{n-1}, n-1\right)$-rectifiable.

Theorem 6.2. Let $u \in \operatorname{GBD}(\Omega)$, let $J_{u}$ be the jump set introduced in Definition 2.4, and let $\Theta_{u}$ be the set defined in (6.1). Then $J_{u}$ is countably $\left(\mathcal{H}^{n-1}, n-1\right)$-rectifiable, $J_{u} \subset \Theta_{u}$, and $\mathcal{H}^{n-1}\left(\Theta_{u} \backslash J_{u}\right)=0$.

Proof. To prove that $J_{u} \subset \Theta_{u}$, let us fix $x_{0} \in J_{u}$. Up to a translation, we may assume that $x_{0}=0$ and that $u^{-}(0)=0$. By Definition 2.4 there exist $a \in \mathbb{R}^{n}$, with $a \neq 0$, and $v \in \mathbb{S}^{n-1}$ such that

$$
\underset{\substack{x \cdot v>0 \\ x \rightarrow 0}}{\operatorname{ap} \lim } u(x)=a \quad \text { and } \quad \underset{\substack{x \cdot v<0 \\ x \rightarrow 0}}{\operatorname{ap} \lim } u(x)=0 .
$$


Let $r>0$ be such that $B_{r}(0) \subset \Omega$. For every $0<\rho<r$ we define $u_{\rho}: B_{1}(0) \rightarrow \mathbb{R}^{n}$ by setting $u_{\rho}(y):=u(\rho y)$ for every $y \in B_{1}(0)$. By a change of variables is easy to see that $u_{\rho} \in \operatorname{GBD}\left(B_{1}(0)\right)$ and that

$$
\hat{\mu}_{u_{\rho}}^{\xi}\left(B_{1}(0)\right)=\frac{\hat{\mu}_{u}^{\xi}\left(B_{\rho}(0)\right)}{\rho^{n-1}}
$$

for every $\xi \in \mathbb{S}^{n-1}$ and for every $0<\rho<r$. By (6.4), $u_{\rho} \rightarrow u_{0}$ in $\mathcal{L}^{n}$-measure on $B_{1}(0)$, where $u_{0}(x)=a$ for $x \cdot v>0$ and $u_{0}(x)=0$ for $x \cdot v<0$. Let us fix $\xi \in \mathbb{S}^{n-1}$ such that $v \cdot \xi \neq 0$ and $0<|a \cdot \xi|<1$. By Remark 4.13 we have $\hat{\mu}_{u_{0}}^{\xi}\left(B_{1}(0)\right)=\omega_{n-1}|v \cdot \xi||a \cdot \xi|$. Therefore (4.8) and (6.5), together with Lemma 4.18, give that

$$
0<\omega_{n-1}|v \cdot \xi||a \cdot \xi| \leq \liminf _{\rho \rightarrow 0+} \frac{\hat{\mu}_{u}^{\xi}\left(B_{\rho}(0)\right)}{\rho^{n-1}} \leq \liminf _{\rho \rightarrow 0+} \frac{\hat{\mu}_{u}\left(B_{\rho}(0)\right)}{\rho^{n-1}} .
$$

This implies that $0 \in \Theta_{u}$ by (6.1), and concludes the proof of the inclusion $J_{u} \subset \Theta_{u}$.

Since $\Theta_{u}$ is countably $\left(\mathcal{H}^{n-1}, n-1\right)$-rectifiable by Proposition 6.1, the rectifiability of $J_{u}$ follows from the inclusion $J_{u} \subset \Theta_{u}$.

Let us prove that $\mathcal{H}^{n-1}\left(\Theta_{u} \backslash J_{u}\right)=0$. It suffices to show that $\mathcal{H}^{n-1}\left(\Theta_{u}^{\varepsilon} \backslash J_{u}\right)=0$ for every $\varepsilon>0$, where $\Theta_{u}^{\varepsilon}$ is the set defined in (6.2). By (6.3) it is enough to prove that $\hat{\mu}_{u}\left(\Theta_{u}^{\varepsilon} \backslash J_{u}\right)=0$, and by (4.8) we have to show that

$$
\hat{\mu}_{u}^{\xi}\left(\Theta_{u}^{\varepsilon} \backslash J_{u}\right)=0 \quad \text { for every } \xi \in \mathbb{S}^{n-1} .
$$

Let us fix $\xi \in \mathbb{S}^{n-1}$. Since $\Theta_{u}^{\varepsilon}$ is countably $\left(\mathcal{H}^{n-1}, n-1\right)$-rectifiable, we can write

$$
\Theta_{u}^{\varepsilon} \backslash J_{u}=N_{0} \cup \bigcup_{i=1}^{\infty} N_{i}
$$

with $\mathcal{H}^{n-1}\left(N_{0}\right)=0$ and $N_{i} \subset M_{i}$ for every $i \geq 1$, where each $M_{i}$ is a $C^{1}$ manifold of dimension $n-1$ with normal unit vector $v_{i}$. We define

$$
M_{i}^{ \pm}:=\left\{x \in M_{i}: \pm \xi \cdot v_{i}(x)>0\right\} \quad \text { and } \quad M_{i}^{0}:=\left\{x \in M_{i}: \xi \cdot v_{i}(x)=0\right\} .
$$

Therefore $N_{i}=N_{i}^{+} \cup N_{i}^{-} \cup N_{i}^{0}$, where $N_{i}^{ \pm}:=N_{i} \cap M_{i}^{ \pm}$and $N_{i}^{0}:=N_{i} \cap M_{i}^{0}$. Since $\mathcal{H}^{n-1}\left(N_{0}\right)=0$ we have $\mathcal{H}^{n-1}\left(\pi^{\xi}\left(N_{0}\right)\right)=0$. By the area formula (see, e.g., [6, Theorem 2.91]) and by (6.8) we have $\mathcal{H}^{n-1}\left(\pi^{\xi}\left(N_{i}^{0}\right)\right)=0$ for every $i$. Therefore (4.5) implies that $\hat{\mu}_{u}^{\xi}\left(N_{0}\right)=0$ and $\hat{\mu}_{u}^{\xi}\left(N_{i}^{0}\right)=0$ for every $i$. It follows from (6.7) that

$$
\hat{\mu}_{u}^{\xi}\left(\Theta_{u}^{\varepsilon} \backslash J_{u}\right) \leq \sum_{i=1}^{\infty} \hat{\mu}^{\xi}\left(N_{i}^{+}\right)+\sum_{i=1}^{\infty} \hat{\mu}^{\xi}\left(N_{i}^{-}\right) .
$$

To prove (6.6) it is enough to show that for every $i$ we have

$$
\hat{\mu}_{u}^{\xi}\left(N_{i}^{+}\right)=0 \quad \text { and } \quad \hat{\mu}_{u}^{\xi}\left(N_{i}^{-}\right)=0
$$


Let us fix $i$ and let $u_{i}^{+}$and $u_{i}^{-}$be the traces of $u$ on $M_{i}$, oriented by $v_{i}$. Splitting $M_{i}^{+}$into a countable number of pieces, we may assume that there exist an open set $A$ in the relative topology of $\Pi^{\xi}$ and a function $\psi \in C^{1}(A)$ such that $M_{i}^{+}=\{y+\psi(y) \xi: y \in A\}$. By (6.8) we have $\xi \cdot v_{i}(x)>0$ for every $x \in M_{i}^{+}$. By Theorem 5.2 for $\mathcal{H}^{n-1}$-a.e. $y \in A$ we have

$$
u_{i}^{-}(y+\psi(y) \xi) \cdot \xi=\operatorname{ap}_{\substack{t \rightarrow \psi(y) \\ t<\psi(y)}} \hat{u}_{y}^{\xi}(t) \quad \text { and } \quad u_{i}^{+}(y+\psi(y) \xi) \cdot \xi=\operatorname{ap}_{\substack{t \rightarrow \psi(y) \\ t>\psi(y)}} \hat{u}_{y}^{\xi}(t)
$$

Since $N_{i}^{+} \cap J_{u}=\emptyset$, we have $u_{i}^{+}(x)=u_{i}^{-}(x)$ for every $x \in N_{i}^{+}$by Remark 5.4. By (6.11) we have

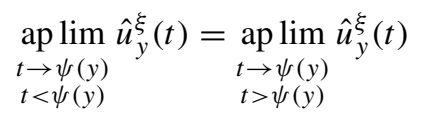

for $\mathcal{H}^{n-1}$-a.e. $y \in B:=\pi^{\xi}\left(N_{i}^{+}\right)$, hence $\psi(y) \notin J_{\hat{u}_{y}^{\xi} \cdot \xi}$ for $\mathcal{H}^{n-1}$-a.e. $y \in B$. Since $N_{i}^{+}=\{y+\psi(y) \xi: y \in B\}$ we have $\left(N_{i}^{+}\right)_{y}^{\xi}=\{\psi(y)\}$ for $y \in B$ and $\left(N_{i}^{+}\right)_{y}^{\xi}=\emptyset$ for $y \in \Pi^{\xi} \backslash B$. Therefore (4.4) and (4.5) give

$$
\hat{\mu}_{u}^{\xi}\left(N_{i}^{+}\right)=\int_{B}\left|D \hat{u}_{y}^{\xi}\right|(\{\psi(y)\}) d \mathcal{H}^{n-1}(y)=0,
$$

since $\psi(y) \notin J_{\hat{u}_{y}^{\xi}}$ for $\mathcal{H}^{n-1}$-a.e. $y \in B$. A similar argument shows that $\hat{\mu}_{u}^{\xi}\left(N_{i}^{-}\right)=0$. This proves (6.10) and concludes the proof of the equality $\mathcal{H}^{n-1}\left(\Theta_{u} \backslash J_{u}\right)=0$.

\section{The jump points of the restriction to hyperplanes}

In this section we prove a technical result that will play a crucial role in the proof of the slicing theorem for the jump set of a GBD function $u$ : all jump points of the restriction of the function $\pi^{\eta}(u)$ to the hyperplane $x_{0}+\Pi^{\eta}$ belong to the set $\Theta_{u}$ introduced in (6.1), provided that $\mathcal{H}^{n-1}\left(S_{u} \cap\left(x_{0}+\Pi^{\eta}\right)\right)=0$.

A key tool in the proof is the following parallelogram identity, which holds for every function $v: \Omega \rightarrow \mathbb{R}^{n}$ :

$$
\begin{aligned}
& v(x+h \xi) \cdot(\xi+\eta)-v(x-h \eta) \cdot(\xi+\eta) \\
+ & v(x+h \eta) \cdot(\xi+\eta)-v(x-h \xi) \cdot(\xi+\eta) \\
+ & v(x+h \xi) \cdot(\xi-\eta)-v(x+h \eta) \cdot(\xi-\eta) \\
+ & v(x-h \eta) \cdot(\xi-\eta)-v(x-h \xi) \cdot(\xi-\eta) \\
& =2 v(x+h \xi) \cdot \xi-2 v(x-h \xi) \cdot \xi+2 v(x+h \eta) \cdot \eta-2 v(x-h \eta) \cdot \eta
\end{aligned}
$$

for every $x \in \Omega$, every $\xi, \eta \in \mathbb{R}^{n}$, and every $h>0$ such that $x \pm h \xi, x \pm h \eta \in \Omega$. 
Theorem 7.1. Let $u \in \operatorname{GBD}(\Omega)$, let $x_{0} \in \Omega$, and let $\eta \in \mathbb{S}^{n-1}$. Assume that

$$
\mathcal{H}^{n-1}\left(S_{u} \cap\left(x_{0}+\Pi^{\eta}\right)\right)=0 \text {. }
$$

Let $v:\left(-x_{0}+\Omega\right) \cap \Pi^{\eta} \rightarrow \Pi^{\eta}$ be the function defined by $v(y):=\pi^{\eta}\left(\tilde{u}\left(x_{0}+y\right)\right)$ for $\mathcal{H}^{n-1}$-a.e. $y \in\left(-x_{0}+\Omega\right) \cap \Pi^{\eta}$. Suppose that there exist $v \in \mathbb{S}^{n-1} \cap \Pi^{\eta}$ and $b^{+}, b^{-} \in \Pi^{\eta}$ such that

$$
\lim _{\rho \rightarrow 0} \frac{\mathcal{H}^{n-1}\left(\left\{y \in B_{\rho}(0) \cap \Pi^{\eta}: \pm y \cdot v>0,\left|v(y)-b^{ \pm}\right|>\varepsilon\right\}\right)}{\rho^{n-1}}=0 .
$$

If $b^{+} \neq b^{-}$, then $x_{0} \in \Theta_{u}$.

Proof. It is not restrictive to consider only the case $x_{0}=0$. We assume, by contradiction, that $b^{+} \neq b^{-}$and $0 \notin \Theta_{u}$, and we fix $\xi \in \mathbb{S}^{n-1} \cap \Pi^{\eta}$ such that

$$
\left|\left(b^{+}-b^{-}\right) \cdot \xi\right| \geq \frac{1}{2}\left|b^{+}-b^{-}\right| \text {and } v \cdot \xi>0 .
$$

Let $S$ be the set of all $s \in \mathbb{R}^{+}$such that $y+s \eta \notin S_{u}$ and $y-s \eta \notin S_{u}$ for $\mathcal{H}^{n-1}$-a.e. $y \in \Pi^{\eta}$. Then $0 \in S$ and $\mathcal{L}^{1}\left(\mathbb{R}^{+} \backslash S\right)=0$ by Fubini's theorem, since $\mathcal{L}^{n}\left(S_{u}\right)=0$ by Remark 2.5.

For every $\rho>0$ we set $B_{\rho}:=B_{\rho}(0), B_{\rho}^{0}:=B_{\rho} \cap \Pi^{\eta}, B_{\rho}^{0 \pm}:=\left\{y \in B_{\rho}^{0}: \pm y \cdot v>0\right\}$, and $A_{\rho}:=B_{c \rho}^{0}=B_{c \rho} \cap \Pi^{\eta}$, with $0<c<v \cdot \xi \leq 1$. It follows that

$$
\rho \xi+A_{\rho} \subset B_{2 \rho}^{0+} \quad \text { and } \quad-\rho \xi+A_{\rho} \subset B_{2 \rho}^{0-} .
$$

Since $0 \notin \Theta_{u}$, by (6.1) we have

$$
\lim _{\rho \rightarrow 0+} \frac{\hat{\mu}_{u}\left(B_{\rho}\right)}{\rho^{n-1}}=0 .
$$

Let us fix $\varepsilon>0$ such that $3 \varepsilon<\frac{1}{2}\left|b^{+}-b^{-}\right|$. By (7.4) for every $\rho>0$ and every $y \in A_{\rho}$ we have

$$
\begin{aligned}
3 \varepsilon & <\left|\left(b^{+}-b^{-}\right) \cdot \xi\right| \\
& \leq\left|b^{+} \cdot \xi-v(y+\rho \xi) \cdot \xi\right|+|(v(y+\rho \xi)-v(y-\rho \xi)) \cdot \xi|+\left|v(y-\rho \xi) \cdot \xi-b^{-} \cdot \xi\right| \\
& \leq\left|b^{+}-v(y+\rho \xi)\right|+|(v(y+\rho \xi)-v(y-\rho \xi)) \cdot \xi|+\left|v(y-\rho \xi)-b^{-}\right| .
\end{aligned}
$$

It follows that

$$
\begin{aligned}
\mathcal{H}^{n-1}\left(A_{\rho}\right) \leq & \mathcal{H}^{n-1}\left(\left\{y \in A_{\rho}:\left|b^{+}-v(y+\rho \xi)\right|>\varepsilon\right\}\right) \\
& +\mathcal{H}^{n-1}\left(\left\{y \in A_{\rho}:|(v(y+\rho \xi)-v(y-\rho \xi)) \cdot \xi|>\varepsilon\right\}\right) \\
& +\mathcal{H}^{n-1}\left(\left\{y \in A_{\rho}:\left|v(y-\rho \xi)-b^{-}\right|>\varepsilon\right\}\right) .
\end{aligned}
$$

To conclude the proof of the theorem it is enough to show that

$$
\mathcal{H}^{n-1}\left(A_{\rho}\right)=o\left(\rho^{n-1}\right)
$$


along a sequence converging to zero. Indeed, the definition of $A_{\rho}$ gives $\mathcal{H}^{n-1}\left(A_{\rho}\right)=$ $\omega_{n-1} c^{n-1} \rho^{n-1}$, which contradicts (7.8) and shows that the relations $b^{+} \neq b^{-}$and $0 \notin \Theta_{u}$ cannot be true simultaneously.

To estimate the first term of the right-hand side of (7.7), we use (7.5) and we obtain

$$
\begin{aligned}
\mathcal{H}^{n-1}\left(\left\{y \in A_{\rho}:\left|b^{+}-v(y+\rho \xi)\right|>\varepsilon\right\}\right) & =\mathcal{H}^{n-1}\left(\left\{x \in \rho \xi+A_{\rho}:\left|b^{+}-v(x)\right|>\varepsilon\right\}\right) \\
& \leq \mathcal{H}^{n-1}\left(\left\{x \in B_{2 \rho}^{0+}:\left|b^{+}-v(x)\right|>\varepsilon\right\}\right) .
\end{aligned}
$$

By (7.3) the last term is $o\left(\rho^{n-1}\right)$, so that

$$
\mathcal{H}^{n-1}\left(\left\{y \in A_{\rho}:\left|b^{+}-v(y+\rho \xi)\right|>\varepsilon\right\}\right)=o\left(\rho^{n-1}\right) .
$$

In the same way we prove that

$$
\mathcal{H}^{n-1}\left(\left\{y \in A_{\rho}:\left|v(y-\rho \xi)-b^{-}\right|>\varepsilon\right\}\right)=o\left(\rho^{n-1}\right) .
$$

It remains to estimate $\mathcal{H}^{n-1}\left(\left\{y \in A_{\rho}:|(v(y+\rho \xi)-v(y-\rho \xi)) \cdot \xi|>\varepsilon\right\}\right)$. Since $\xi \in \Pi^{\eta}$, we have $v(y) \cdot \xi=\tilde{u}(y) \cdot \xi$ for $\mathcal{H}^{n-1}$-a.e. $y \in \Pi^{\eta}$, hence

$$
\begin{aligned}
\mathcal{H}^{n-1}\left(\left\{y \in A_{\rho}:\right.\right. & |(v(y+\rho \xi)-v(y-\rho \xi)) \cdot \xi|>\varepsilon\}) \\
= & \mathcal{H}^{n-1}\left(\left\{y \in A_{\rho}:|(\tilde{u}(y+\rho \xi)-\tilde{u}(y-\rho \xi)) \cdot \xi|>\varepsilon\right\}\right) .
\end{aligned}
$$

By the parallelogram identity (7.1) we have

$$
\begin{aligned}
\mathcal{H}^{n-1} & \left(\left\{y \in A_{\rho}:|(\tilde{u}(y+\rho \xi)-\tilde{u}(y-\rho \xi)) \cdot \xi|>\varepsilon\right\}\right) \\
& \leq \mathcal{H}^{n-1}\left(\left\{y \in A_{\rho}:|\tilde{u}(y+\rho \eta) \cdot \eta-\tilde{u}(y-\rho \eta) \cdot \eta|>\varepsilon / 5\right\}\right) \\
& +\mathcal{H}^{n-1}\left(\left\{y \in A_{\rho}:|\tilde{u}(y+\rho \xi) \cdot(\xi+\eta)-\tilde{u}(y-\rho \eta) \cdot(\xi+\eta)|>2 \varepsilon / 5\right\}\right) \\
& +\mathcal{H}^{n-1}\left(\left\{y \in A_{\rho}:|\tilde{u}(y+\rho \eta) \cdot(\xi+\eta)-\tilde{u}(y-\rho \xi) \cdot(\xi+\eta)|>2 \varepsilon / 5\right\}\right) \\
& +\mathcal{H}^{n-1}\left(\left\{y \in A_{\rho}:|\tilde{u}(y+\rho \xi) \cdot(\xi-\eta)-\tilde{u}(y+\rho \eta) \cdot(\xi-\eta)|>2 \varepsilon / 5\right\}\right) \\
& +\mathcal{H}^{n-1}\left(\left\{y \in A_{\rho}:|\tilde{u}(y-\rho \eta) \cdot(\xi-\eta)-\tilde{u}(y-\rho \xi) \cdot(\xi-\eta)|>2 \varepsilon / 5\right\}\right) .
\end{aligned}
$$

To estimate the first term on the right-hand side we fix $\tau \in C^{1}(\mathbb{R})$ with $-1 / 2<$ $\tau(t)<1 / 2,0<\tau^{\prime}(t)<1$, and $\tau(-t)=-\tau(t)$ for every $t \in \mathbb{R}$. Since $\tau$ is increasing, we have

$$
\begin{aligned}
& \mathcal{H}^{n-1}\left(\left\{y \in A_{\rho}:|\tilde{u}(y+\rho \eta) \cdot \eta-\tilde{u}(y-\rho \eta) \cdot \eta|>\varepsilon / 5\right\}\right) \\
& \quad=\mathcal{H}^{n-1}\left(\left\{y \in A_{\rho}:|\tau(\tilde{u}(y+\rho \eta) \cdot \eta-\tilde{u}(y-\rho \eta) \cdot \eta)|>\tau(\varepsilon / 5)\right\}\right) .
\end{aligned}
$$

Let $r: \mathbb{R}^{n} \rightarrow \mathbb{R}^{n}$ be the reflection in $\Pi^{\eta}$ :

$$
r(x):=x-2(x \cdot \eta) \eta
$$

for every $x \in \mathbb{R}^{n}$. Let $\hat{\Omega}:=\Omega \cap r(\Omega)$ and let $\varphi \in L^{\infty}(\hat{\Omega})$ be the function defined by

$$
\varphi(x):=\tau(u(x) \cdot \eta-u(r(x)) \cdot \eta) .
$$


If $x \in \hat{\Omega} \backslash S_{u}$ and $r(x) \notin S_{u}$, then $x \notin S_{\varphi}$ and

$$
\tilde{\varphi}(x)=\tau(\tilde{u}(x) \cdot \eta-\tilde{u}(r(x)) \cdot \eta) .
$$

For every $y \in \Pi^{\eta}$ we have

$$
\varphi_{y}^{\eta}=\tau\left(\hat{u}_{y}^{\eta}-\check{u}_{y}^{\eta}\right) \quad \text { on } \hat{\Omega}_{y}^{\eta},
$$

where $\check{u}_{y}^{\eta}(t):=\hat{u}_{y}^{\eta}(-t)=u(y-t \eta) \cdot \eta$.

By condition (b) of Definition 4.1 we have $\hat{u}_{y}^{\eta} \in \mathrm{BV}_{\mathrm{loc}}\left(\hat{\Omega}_{y}^{\eta}\right)$ and $\check{u}_{y}^{\eta} \in \mathrm{BV}_{\text {loc }}\left(\hat{\Omega}_{y}^{\eta}\right)$ for $\mathcal{H}^{n-1}$-a.e. $y \in \Pi^{\eta}$. By (7.15) this implies that $\varphi_{y}^{\eta} \in \mathrm{BV}_{\mathrm{loc}}\left(\hat{\Omega}_{y}^{\eta}\right)$ for $\mathcal{H}^{n-1}$-a.e. $y \in \Pi^{\eta}$. Since $0<\tau^{\prime}<1$ and $-1 / 2<\tau<1 / 2$, arguing as in the proof of Proposition 3.4 we deduce from Vol'pert's chain rule in BV (see [6, Theorem 3.96]) that

$$
\begin{aligned}
\left|D \varphi_{y}^{\eta}\right|\left(B \backslash J_{\varphi_{y}^{\eta}}\right) \leq & \left|D \hat{u}_{y}^{\eta}\right|\left(B \backslash J_{\hat{u}_{y}^{\eta}}\right)+\left|D \check{u}_{y}^{\eta}\right|\left(B \backslash J_{\check{u}_{y}^{\eta}}\right), \\
\left|D \varphi_{y}^{\eta}\right|\left(B \cap J_{\varphi_{y}^{\eta}}\right) \leq & \left|D \hat{u}_{y}^{\eta}\right|\left(B \cap J_{\hat{u}_{y}^{\eta}} \backslash J_{\hat{u}_{y}^{\eta}}^{1}\right)+\mathcal{H}^{0}\left(B \cap J_{\hat{u}_{y}^{\eta}}^{1}\right) \\
& +\left|D \hat{u}_{y}^{\eta}\right|\left(B \cap J_{\check{u}_{y}^{\eta}} \backslash J_{\check{u}_{y}^{\eta}}^{1}\right)+\mathcal{H}^{0}\left(B \cap J_{\check{u}_{y}^{\eta}}^{1}\right)
\end{aligned}
$$

for $\mathcal{H}^{n-1}$-a.e. $y \in \Pi^{\eta}$ and for every Borel set $B \subset \hat{\Omega}_{y}^{\eta}$. By an easy change of variables we obtain from the previous inequalities and from (4.4)

$$
\begin{aligned}
\left|D \varphi_{y}^{\eta}\right|(B) \leq & \left|D \hat{u}_{y}^{\eta}\right|\left(B \backslash J_{\hat{u}_{y}^{\eta}}^{1}\right)+\mathcal{H}^{0}\left(B \cap J_{\hat{u}_{y}^{\eta}}\right) \\
& +\left|D \hat{u}_{y}^{\eta}\right|\left((-B) \backslash J_{\hat{u}_{y}^{\eta}}^{1}\right)+\mathcal{H}^{0}\left((-B) \cap J_{\hat{u}_{y}^{\eta}}\right) \leq 2 \mu_{y}^{\eta}(B \cup(-B)) .
\end{aligned}
$$

Integrating on $\Pi^{\eta}$ we get

$$
\int_{\Pi^{\eta}}\left|D \varphi_{y}^{\eta}\right|\left(\hat{\Omega}_{y}^{\eta}\right) d \mathcal{H}^{n-1}(y) \leq 2 \int_{\Pi^{\eta}} \mu_{y}^{\eta}\left(\hat{\Omega}_{y}^{\eta}\right) d \mathcal{H}^{n-1}(y)=2 \mu^{\eta}(\hat{\Omega})<\infty,
$$

so that $D_{\eta} \varphi \in \mathcal{M}_{b}(\hat{\Omega})$ by Proposition 3.1.

Let us fix $\rho \in S$ with $B_{2 \rho} \subset \Omega$. Since $y \notin S_{u}$ and $y \pm \rho \eta \notin S_{u}$ for $\mathcal{H}^{n-1}$-a.e. $y \in \Pi^{\eta}$, while $y \pm \rho \eta \in B_{2 \rho} \subset \Omega$ for every $y \in A_{\rho}$, we have $y \in \hat{\Omega} \backslash S_{\varphi}$ and $y \pm \rho \eta \in \hat{\Omega} \backslash S_{\varphi}$ for $\mathcal{H}^{n-1}$-a.e. $y \in A_{\rho}$. Moreover $\tilde{\varphi}(y)=0$ for $\mathcal{H}^{n-1}$-a.e. $y \in \Omega \cap \Pi^{\eta}$ by (7.14). We can now apply Proposition 3.2 to obtain

$$
|\tilde{\varphi}(y+\rho \eta)|=|\tilde{\varphi}(y+\rho \eta)-\tilde{\varphi}(y)| \leq\left(V \tilde{\varphi}_{y}^{\eta}\right)([0, \rho])=\left|D \varphi_{y}^{\eta}\right|([0, \rho])
$$

for $\mathcal{H}^{n-1}$-a.e. $y \in A_{\rho}$. Therefore (7.16) yields

$$
|\tilde{\varphi}(y+\rho \eta)| \leq 2 \mu_{y}^{\eta}([-\rho, \rho]) .
$$

Integrating over $A_{\rho}$ we get

$$
\int_{A_{\rho}}|\tilde{\varphi}(y+\rho \eta)| d \mathcal{H}^{n-1}(y) \leq 2 \int_{A_{\rho}} \mu_{y}^{\eta}([-\rho, \rho]) d \mathcal{H}^{n-1}(y) .
$$


Since $y+t \eta \in B_{2 \rho}$ for every $y \in A_{\rho}$ and every $t \in[-\rho, \rho]$, by (4.5) we get

$$
\int_{A_{\rho}} \mu_{y}^{\eta}([-\rho, \rho]) d \mathcal{H}^{n-1}(y) \leq \mu^{\eta}\left(B_{2 \rho}\right) .
$$

From (4.8), (7.18), and (7.19) we deduce that

$$
\int_{A_{\rho}}|\tilde{\varphi}(y+\rho \eta)| d \mathcal{H}^{n-1}(y) \leq 2 \mu^{\eta}\left(B_{2 \rho}\right) \leq 2 \mu_{u}\left(B_{2 \rho}\right) .
$$

Since $y \pm \rho \eta \in \Omega \backslash S_{u}$ for $\mathcal{H}^{n-1}$-a.e. $y \in A_{\rho}$, by (7.14) we have $\tau(\tilde{u}(y+\rho \eta) \cdot \eta-$ $\tilde{u}(y-\rho \eta) \cdot \eta)=\tilde{\varphi}(y+\rho \eta)$ for $\mathcal{H}^{n-1}$-a.e. $y \in A_{\rho}$. Therefore (7.13), (7.20), and Chebyshev's inequality give

$$
\begin{aligned}
\mathcal{H}^{n-1}\left(\left\{y \in A_{\rho}: \mid \tilde{u}(y\right.\right. & +\rho \eta) \cdot \eta-\tilde{u}(y-\rho \eta) \cdot \eta \mid>\varepsilon / 5\}) \\
& =\mathcal{H}^{n-1}\left(\left\{y \in A_{\rho}:|\tilde{\varphi}(y+\rho \eta)|>\tau(\varepsilon / 5)\right\}\right) \\
& \leq \frac{1}{\tau(\varepsilon / 5)} \int_{A_{\rho}}|\tilde{\varphi}(y+\rho \eta)| d \mathcal{H}^{n-1}(y) \leq \frac{2}{\tau(\varepsilon / 5)} \mu_{u}\left(B_{2 \rho}\right)
\end{aligned}
$$

By (7.6) this implies that

$$
\mathcal{H}^{n-1}\left(\left\{y \in A_{\rho}:|\tilde{u}(y+\rho \eta) \cdot \eta-\tilde{u}(y-\rho \eta) \cdot \eta|>\varepsilon / 5\right\}\right)=o\left(\rho^{n-1}\right) \quad \text { for } \rho \in S .
$$

To estimate the second term on the right-hand side of (7.12) we set $\omega:=(\xi+\eta) / \sqrt{2}$ and we replace the reflection $r$ by the involution

$$
q(x):=x-2 \sqrt{2}(x \cdot \eta) \omega-\sqrt{2} \rho \omega=x-2(x \cdot \eta)(\xi+\eta)-\rho(\xi+\eta),
$$

which leaves the hyperplane $-\frac{\rho}{2} \eta+\Pi^{\eta}$ fixed and moves all points in the direction determined by $\omega$. We now define $\tilde{\Omega}:=\Omega \cap q(\Omega)$ and $\psi \in L^{\infty}(\tilde{\Omega})$ by

$$
\psi(x):=\tau(u(x) \cdot(\xi+\eta)-u(q(x)) \cdot(\xi+\eta)) .
$$

If $x \in \tilde{\Omega} \backslash S_{u}$ and $q(x) \notin S_{u}$, then $x \notin S_{\psi}$ and

$$
\tilde{\psi}(x)=\tau(\tilde{u}(x) \cdot(\xi+\eta)-\tilde{u}(q(x)) \cdot(\xi+\eta)) .
$$

For every $y \in-\frac{\rho}{2} \eta+\Pi^{\eta}$ we have $\psi_{y}^{\omega}=\tau\left(\sqrt{2} \hat{u}_{y}^{\omega}-\sqrt{2} \check{u}_{y}^{\omega}\right)$ on $\tilde{\Omega}_{y}^{\omega}$, where $\check{u}_{y}^{\omega}(t):=$ $\hat{u}_{y}^{\omega}(-t)=u(y-t \omega) \cdot \omega$.

Arguing as in the previous step we now obtain

$$
\left|D \psi_{y}^{\omega}\right|(B) \leq 2 \sqrt{2} \mu_{y}^{\omega}(B \cup(-B))
$$


for $\mathcal{H}^{n-1}$-a.e. $y \in-\frac{\rho}{2} \eta+\Pi^{\eta}$ and for every Borel set $B \subset \tilde{\Omega}_{y}^{\omega}$. Integrating on $\Pi^{\omega}$ and $-\frac{\rho}{2} \eta+\Pi^{\eta}$ we obtain, thanks to Remark 4.11,

$$
\begin{aligned}
\int_{\Pi^{\omega}}\left|D \psi_{y}^{\omega}\right|\left(\tilde{\Omega}_{y}^{\omega}\right) d \mathcal{H}^{n-1}(y) & =\frac{1}{\sqrt{2}} \int_{-\frac{\rho}{2} \eta+\Pi^{\eta}}\left|D \psi_{y}^{\omega}\right|\left(\tilde{\Omega}_{y}^{\omega}\right) d \mathcal{H}^{n-1}(y) \\
& \leq 2 \int_{-\frac{\rho}{2} \eta+\Pi^{\eta}} \mu_{y}^{\omega}\left(\tilde{\Omega}_{y}^{\omega}\right) d \mathcal{H}^{n-1}(y)=2 \sqrt{2} \mu^{\omega}(\tilde{\Omega})<\infty,
\end{aligned}
$$

which gives $D_{\omega} \psi \in \mathcal{M}_{b}(\tilde{\Omega})$ thanks to Proposition 3.1.

Let us fix $\rho \in S$ with $\rho / 2 \in S$ and $B_{2 \rho} \subset \Omega$. For every $y \in \Pi^{\eta}$ we define $a(y):=$ $y+\frac{\rho}{2}(\xi-\eta) \in-\frac{\rho}{2} \eta+\Pi^{\eta}$, so that

$$
\begin{aligned}
& y+\rho \xi=a(y)+\frac{\rho}{2}(\xi+\eta)=a(y)+\frac{\rho}{\sqrt{2}} \omega, \\
& y-\rho \eta=a(y)-\frac{\rho}{2}(\xi+\eta)=a(y)-\frac{\rho}{\sqrt{2}} \omega .
\end{aligned}
$$

Since $y+\rho \xi \notin S_{u}, y-\rho \eta \notin S_{u}$, and $a(y) \notin S_{u}$ for $\mathcal{H}^{n-1}$-a.e. $y \in \Pi^{\eta}$, while $y+\rho \xi \in$ $B_{2 \rho} \subset \Omega, y-\rho \eta \in B_{2 \rho} \subset \Omega$, and $a(y) \in B_{2 \rho} \subset \Omega$ for every $y \in A_{\rho}$, we have $y+\rho \xi \in \tilde{\Omega} \backslash S_{\psi}, y-\rho \eta \in \tilde{\Omega} \backslash S_{\psi}$, and $a(y) \in \tilde{\Omega} \backslash S_{\psi}$ for $\mathcal{H}^{n-1}$-a.e. $y \in A_{\rho}$. Moreover $\tilde{\psi}(a(y))=0$ for $\mathcal{H}^{n-1}$-a.e. $y \in A_{\rho}$ by $(7.21)$, since $q(a(y))=a(y)$ for every $y \in \Pi^{\eta}$. We can now apply Proposition 3.2 to obtain

$$
\begin{aligned}
|\tilde{\psi}(y+\rho \xi)| & =\left|\tilde{\psi}\left(a(y)+\frac{\rho}{\sqrt{2}} \omega\right)-\tilde{\psi}(a(y))\right| \leq\left(V \tilde{\psi}_{a(y)}^{\omega}\right)([0, \rho / \sqrt{2}]) \\
& =\left|D \psi_{a(y)}^{\omega}\right|([0, \rho / \sqrt{2}])
\end{aligned}
$$

for $\mathcal{H}^{n-1}$-a.e. $y \in A_{\rho}$. Therefore (7.22) yields

$$
|\tilde{\psi}(y+\rho \xi)| \leq 2 \sqrt{2} \mu_{a(y)}^{\omega}([-\rho / \sqrt{2}, \rho / \sqrt{2}]) .
$$

Integrating over $A_{\rho}$ we get

$$
\left.\int_{A_{\rho}} \mid \tilde{\psi}(y+\rho \xi)\right) \mid d \mathcal{H}^{n-1}(y) \leq 2 \sqrt{2} \int_{A_{\rho}} \mu_{a(y)}^{\omega}([-\rho / \sqrt{2}, \rho / \sqrt{2}]) d \mathcal{H}^{n-1}(y) .
$$

Since $a(y)+t \omega \in B_{2 \rho}$ for every $y \in A_{\rho}$ and for every $t \in[-\rho / \sqrt{2}, \rho / \sqrt{2}]$, by Remark 4.11 we get

$$
\int_{A_{\rho}} \mu_{a(y)}^{\omega}([-\rho / \sqrt{2}, \rho / \sqrt{2}]) \mid d \mathcal{H}^{n-1}(y) \leq \sqrt{2} \mu^{\omega}\left(B_{2 \rho}\right) .
$$

From (4.8), (7.24), and (7.25) we deduce that

$$
\int_{A_{\rho}}|\tilde{\psi}(y+\rho \xi)| d \mathcal{H}^{n-1}(y) \leq 4 \mu^{\omega}\left(B_{2 \rho}\right) \leq 4 \mu_{u}\left(B_{2 \rho}\right) .
$$


Since $y+\rho \xi \in \Omega \backslash S_{u}$ and $q(y+\rho \xi)=y-\rho \eta \in \Omega \backslash S_{u}$ for $\mathcal{H}^{n-1}$-a.e. $y \in A_{\rho}$, by (7.21) we have $\tau(\tilde{u}(y+\rho \xi) \cdot(\xi+\eta)-\tilde{u}(y-\rho \eta) \cdot(\xi+\eta))=\tilde{\psi}(y+\rho \xi)$ for $\mathcal{H}^{n-1}$-a.e. $y \in A_{\rho}$. Therefore (7.26) and Chebyshev's inequality give

$$
\begin{aligned}
\mathcal{H}^{n-1}( & \left.\left\{y \in A_{\rho}:|\tilde{u}(y+\rho \xi) \cdot(\xi+\eta)-\tilde{u}(y-\rho \eta) \cdot(\xi+\eta)|>2 \varepsilon / 5\right\}\right) \\
& =\mathcal{H}^{n-1}\left(\left\{y \in A_{\rho}:|\tau(\tilde{u}(y+\rho \xi) \cdot(\xi+\eta)-\tilde{u}(y-\rho \eta) \cdot(\xi+\eta))|>\tau(2 \varepsilon / 5)\right\}\right) \\
& =\mathcal{H}^{n-1}\left(\left\{y \in A_{\rho}:|\tilde{\psi}(y+\rho \xi)|>\tau(2 \varepsilon / 5)\right\}\right) \\
& \leq \frac{1}{\tau(2 \varepsilon / 5)} \int_{A_{\rho}}|\tilde{\psi}(y+\rho \xi)| d \mathcal{H}^{n-1}(y) \leq \frac{4}{\tau(\varepsilon / 5)} \mu_{u}\left(B_{2 \rho}\right) .
\end{aligned}
$$

By (7.6) this implies that

$$
\mathcal{H}^{n-1}\left(\left\{y \in A_{\rho}:|\tilde{u}(y+\rho \xi) \cdot(\xi+\eta)-\tilde{u}(y-\rho \eta) \cdot(\xi+\eta)|>\varepsilon / 5\right\}\right)=o\left(\rho^{n-1}\right)
$$

for $\rho \in S$ with $\rho / 2 \in S$.

The other terms on the right-hand side of (7.12) can be estimated in a similar way. This proves (7.8) and concludes the proof of the theorem.

\section{Slicing of the jump set}

In this section we prove that for every $u \in \operatorname{GBD}(\Omega)$ the jump set $J_{u}$ introduced in Definition 2.4 can be reconstructed from the jump sets of the one-dimensional slices $\hat{u}_{y}^{\xi}$.

Theorem 8.1. Let $u \in \operatorname{GBD}(\Omega)$, let $\xi \in \mathbb{S}^{n-1}$, and let

$$
J_{u}^{\xi}:=\left\{x \in J_{u}:[u](x) \cdot \xi \neq 0\right\} .
$$

Then for $\mathcal{H}^{n-1}$-a.e. $y \in \Pi^{\xi}$ we have

$$
\begin{gathered}
\left(J_{u}^{\xi}\right)_{y}^{\xi}=J_{\hat{u}_{y}^{\xi}}, \\
u^{ \pm}(y+t \xi) \cdot \xi=\left(\hat{u}_{y}^{\xi}\right)^{ \pm}(t) \quad \text { for every } t \in\left(J_{u}\right)_{y}^{\xi},
\end{gathered}
$$

where the normals to $J_{u}$ and $J_{\hat{u}_{y}^{\xi}}$ are oriented so that $\xi \cdot v_{u} \geq 0$ and $v_{\hat{u}_{y}^{\xi}}=1$.

Proof. Let us prove that for $\mathcal{H}^{n-1}$-a.e. $y \in \Pi^{\xi}$ we have

$$
\left(J_{u}^{\xi}\right)_{y}^{\xi} \subset J_{\hat{u}_{y}^{\xi}} \text {. }
$$

Since $J_{u}$ is countably $\left(\mathcal{H}^{n-1}, n-1\right)$-rectifiable by Theorem 6.2 , we can write

$$
J_{u}=N_{0} \cup \bigcup_{i=1}^{\infty} N_{i},
$$


with $\mathcal{H}^{n-1}\left(N_{0}\right)=0$ and $N_{i} \subset M_{i}$ for every $i \geq 1$, where each $M_{i}$ is a $C^{1}$ manifold of dimension $n-1$ with normal unit vector $v_{i}$. By Remark 5.4 we have $v_{u}= \pm v_{i} \mathcal{H}^{n-1}$ a.e. on $N_{i}$ for every $i \geq 1$. Removing an $\mathcal{H}^{n-1}$-negligible set, we may assume that these equalities hold everywhere on $N_{i}$. Splitting, if needed, each $N_{i}$ into two parts, we may also assume that the sign is constant in each $N_{i}$, and we may reorient the manifold $M_{i}$ so that $v_{u}=v_{i}$ on $N_{i}$ for every $i \geq 1$.

Let $M_{i}^{ \pm}$and $M_{i}^{0}$ be the sets defined in (6.8). Since $\xi \cdot v_{i}=\xi \cdot v_{u} \geq 0$ on $N_{i}$, we have $N_{i}=N_{i}^{+} \cup N_{i}^{0}$, where $N_{i}^{+}:=N_{i} \cap M_{i}^{+}$and $N_{i}^{0}:=N_{i} \cap M_{i}^{0}$. Since $\mathcal{H}^{n-1}\left(N_{0}\right)=0$ we have $\mathcal{H}^{n-1}\left(\pi^{\xi}\left(N_{0}\right)\right)=0$. By the area formula (see, e.g., [6, Theorem 2.91]) and by (6.8) we have $\mathcal{H}^{n-1}\left(\pi^{\xi}\left(N_{i}^{0}\right)\right)=0$ for every $i$. Let $E_{0}$ be the union of the sets $\pi^{\xi}\left(N_{0}\right)$ and $\pi^{\xi}\left(N_{i}^{0}\right)$ for $i \geq 1$. Then $\mathcal{H}^{n-1}\left(E_{0}\right)=0$ and it is enough to prove (8.4) for $\mathcal{H}^{n-1}$-a.e. $y \in \Pi^{\xi} \backslash E_{0}$.

To obtain this result it suffices to show that for every $i \geq 1$ we have

$$
\left(N_{i}^{+}\right)_{y}^{\xi} \cap\left(J_{u}^{\xi}\right)_{y}^{\xi} \subset J_{\hat{u}_{y}^{\xi}}
$$

for $\mathcal{H}^{n-1}$-a.e. $y \in \Pi^{\xi}$. Let us fix $i \geq 1$ and let $u_{i}^{+}$and $u_{i}^{-}$be the traces of $u$ on $M_{i}$, oriented by $v_{i}$. Splitting $M_{i}^{+}$into a countable number of pieces, we may assume that there exist an open set $A$ in the relative topology of $\Pi^{\xi}$ and a function $\psi \in C^{1}(A)$ such that $M_{i}^{+}=\{y+\psi(y) \xi: y \in A\}$. By (6.8) we have $\xi \cdot v_{i}(x)>0$ for every $x \in M_{i}^{+}$. By Theorem 5.2 for $\mathcal{H}^{n-1}$-a.e. $y \in A$ we have

$$
u_{i}^{-}(y+\psi(y) \xi) \cdot \xi=\operatorname{ap}_{\substack{t \rightarrow \psi(y) \\ t<\psi(y)}} \hat{u}_{y}^{\xi}(t) \text { and } \quad u_{i}^{+}(y+\psi(y) \xi) \cdot \xi=\operatorname{ap}_{\substack{t \rightarrow \psi(y) \\ t>\psi(y)}} \hat{u}_{y}^{\xi}(t) .
$$

By Remark 5.4 we have $u_{i}^{+}(x) \cdot \xi=u^{+}(x) \cdot \xi \neq u^{-}(x) \cdot \xi=u_{i}^{-}(x) \cdot \xi$ for $\mathcal{H}^{n-1}$-a.e. $x \in N_{i}^{+} \cap J_{u}^{\xi}$. This inequality, together with (8.7), gives

$$
\operatorname{aplim}_{\substack{t \rightarrow \psi(y) \\ t<\psi(y)}} \hat{u}_{y}^{\xi}(t) \neq \underset{\substack{t \rightarrow \psi(y) \\ t>\psi(y)}}{\operatorname{ap} \lim _{y}} \hat{u}_{y}^{\xi}(t) \quad \text { for } \mathcal{H}^{n-1} \text {-a.e. } y \in B:=\pi^{\xi}\left(N_{i}^{+} \cap J_{u}^{\xi}\right),
$$

hence $\psi(y) \in J_{\hat{u}_{y}^{\xi}}$ for $\mathcal{H}^{n-1}$-a.e. $y \in B$. Since $N_{i}^{+} \cap J_{u}^{\xi}=\{y+\psi(y) \xi: y \in B\}$ we have $\left(N_{i}^{+}\right)_{y}^{\xi} \cap\left(J_{u}^{\xi}\right)_{y}^{\xi}=\{\psi(y)\}$ for $y \in B$ and $\left(N_{i}^{+}\right)_{y}^{\xi} \cap\left(J_{u}^{\xi}\right)_{y}^{\xi}=\emptyset$ for $y \in \Pi^{\xi} \backslash B$. Therefore we have $\left(N_{i}^{+}\right)_{y}^{\xi} \cap\left(J_{u}^{\xi}\right)_{y}^{\xi} \subset J_{\hat{u}_{y}^{\xi}}$ for $\mathcal{H}^{n-1}$-a.e. $y \in \Pi^{\xi}$. This proves (8.6) and concludes the proof of (8.4). Moreover (8.7), together with the equality $u_{i}^{+}(x)=u^{+}(x)$ and $u_{i}^{-}(x)=u^{-}(x)$ for $\mathcal{H}^{n-1}$-a.e. $x \in N_{i}^{+}$(see Remark 5.4), proves (8.3) for $\mathcal{H}^{n-1}$-a.e. $y \in \Pi^{\xi}$.

Let us prove that

$$
J_{\hat{u}_{y}^{\xi}} \subset\left(J_{u}\right)_{y}^{\xi} \quad \text { for } \mathcal{H}^{n-1} \text {-a.e. } y \in \Pi^{\xi} .
$$

This inclusion is trivial for $n=1$. We prove it by induction on the dimension $n$. By changing $u$ on a set of Lebesgue measure zero, we may assume that $u$ is a Borel function 
and that $\hat{u}_{y}^{\xi} \in \operatorname{BV}_{\text {loc }}\left(\Omega_{y}^{\xi}\right)$ for every $y \in \Pi^{\xi}$. Since $\mathcal{H}^{n-1}\left(\Theta_{u} \backslash J_{u}\right)=0$ by Theorem 6.2, to prove (8.8) it is enough to show that

$$
J_{\hat{u}_{y}^{\xi}} \subset\left(\Theta_{u}\right)_{y}^{\xi} \quad \text { for } \mathcal{H}^{n-1} \text {-a.e. } y \in \Pi^{\xi} .
$$

Let $n \geq 2$ and assume that (8.8) is true in dimension $n-1$. We fix $\eta \in \mathbb{S}^{n-1}$ with $\eta \cdot \xi=0$. For every $s \in \mathbb{R}$ and for every $B \subset \Omega$ let $B_{s}:=\left\{z \in \Pi^{\eta}: z+s \eta \in B\right\}$ and let $u_{s}: \Omega_{s} \rightarrow \Pi^{\eta}$ be the function defined by $u_{s}(z):=\pi^{\eta}(u(z+s \eta))$. Then $(s, z) \mapsto u_{s}(z)$ is a Borel function on the open set $\tilde{\Omega}:=\left\{(s, z): s \in \mathbb{R}, z \in \Omega_{s}\right\} \subset \mathbb{R} \times \Pi^{\eta}$.

Let $\tilde{F}:=\left\{(s, z): s \in \mathbb{R}, z \in J_{u_{s}}\right\} \subset \tilde{\Omega}$ and let $F:=\left\{z+s \eta: s \in \mathbb{R}, z \in J_{u_{s}}\right\}=$ $\{z+s \eta:(s, z) \in \tilde{F}\} \subset \Omega$, so that

$$
J_{u_{s}}=F_{s}:=\left\{z \in \Pi^{\eta}: z+s \eta \in F\right\} .
$$

Arguing as in the proof of [6, Proposition 3.69] and using Remark 2.2 we find that $\tilde{F}$ is a Borel subset of $\tilde{\Omega}$, hence $F$ is a Borel subset of $\Omega$.

Let $\Pi^{\eta \xi}:=\Pi^{\eta} \cap \Pi^{\xi}$. Since $\eta \cdot \xi=0$, we have

$$
\Pi^{\xi}=\left\{a+s \eta: a \in \Pi^{\eta \xi}, s \in \mathbb{R}\right\}
$$

and

$$
B_{a+s \eta}^{\xi}=\left(B_{s}\right)_{a}^{\xi}
$$

for every $a \in \Pi^{\eta \xi}$, every $s \in \mathbb{R}$, and every $B \subset \Omega$. Since $u \cdot \xi=\pi^{\eta}(u) \cdot \xi$, we have

$$
\hat{u}_{a+s \eta}^{\xi}=\left(\widehat{u_{s}}\right)_{a}^{\xi} \quad \text { on } \Omega_{a+s \eta}^{\xi}=\left(\Omega_{s}\right)_{a}^{\xi}
$$

for every $a \in \Pi^{\eta \xi}$ and every $s \in \mathbb{R}$.

For every $x \in \Omega$ we can define

$\hat{u}_{+}^{\xi}(x):=\limsup _{\rho \rightarrow 0+} \frac{1}{\rho} \int_{0}^{\rho} u(x+s \xi) \cdot \xi d s$ and $\hat{u}_{-}^{\xi}(x):=\limsup _{\rho \rightarrow 0+} \frac{1}{\rho} \int_{-\rho}^{0} u(x+s \xi) \cdot \xi d s$.

Since we are assuming that $u$ is a Borel function, by Fubini's theorem $\hat{u}_{+}^{\xi}$ and $\hat{u}_{-}^{\xi}$ are Borel functions on $\Omega$. Therefore $E:=\left\{x \in \Omega: \hat{u}_{+}^{\xi}(x) \neq \hat{u}_{-}^{\xi}(x)\right\}$ is a Borel set. For every $y \in \Pi^{\xi}$ we have $\left(\hat{u}_{+}^{\xi}\right)_{y}^{\xi}=\left(\hat{u}_{-}^{\xi}\right)_{y}^{\xi}=\hat{u}_{y}^{\xi} \mathcal{L}^{1}$-a.e. in $\Omega_{y}^{\xi}$ thanks to Lebesgue's differentiation theorem. By elementary properties of $\mathrm{BV}$ functions in dimension one, this implies that

$$
J_{\hat{u}_{y}^{\xi}}=E_{y}^{\xi} \quad \text { for every } y \in \Pi^{\xi} .
$$

By Theorem 4.19 there exists a Borel set $N_{1} \subset \mathbb{R}$, with $\mathcal{L}^{1}\left(N_{1}\right)=0$, such that for every $s \in \mathbb{R} \backslash N_{1}$ the function $u_{s}$ belongs to $\operatorname{GBD}\left(\Omega_{s}\right)$. Moreover, since $\mathcal{L}^{n}\left(S_{u}\right)=0$ and $u=\tilde{u} \mathcal{L}^{n}$-a.e. in $\Omega$ by Remark 2.5, using Fubini's theorem we find a Borel set $N_{2} \subset \mathbb{R}$, with $\mathcal{L}^{1}\left(N_{2}\right)=0$, such that for every $s \in \mathbb{R} \backslash N_{2}$ we have $\mathcal{H}^{n-1}\left(S_{u} \cap\left(s \eta+\Pi^{\eta}\right)\right)=0$ and $u=\tilde{u} \mathcal{H}^{n-1}$-a.e. in $s \eta+\Pi^{\eta}$. Let $N_{0}:=N_{1} \cup N_{2}$. 
By the inductive hypothesis for every $s \in \mathbb{R} \backslash N_{0}$ we have $J_{\left(\widehat{u_{s}}\right)_{a}^{\xi}} \subset\left(J_{u_{s}}\right)_{a}^{\xi}$ for $\mathcal{H}^{n-2}$ a.e. $a \in \Pi^{\eta \xi}:=\Pi^{\eta} \cap \Pi^{\xi}$. By (8.10) and (8.12)-(8.14) we have

$$
\left.E_{a+s \eta}^{\xi}=J_{\hat{u}_{a+s \eta}^{\xi}}=J_{\left(\widehat{u_{s}}\right)}\right)_{a}^{\xi} \subset\left(J_{u_{s}}\right)_{a}^{\xi}=\left(F_{s}\right)_{a}^{\xi}=F_{a+s \eta}^{\xi}
$$

for every $s \in \mathbb{R} \backslash N_{0}$ and for $\mathcal{H}^{n-2}$-a.e. $a \in \Pi^{\eta \xi}$. By (8.11) and Fubini's theorem there exists a Borel set $N \subset \Pi^{\xi}$, with $\mathcal{H}^{n-1}(N)=0$, such that for every $y \in \Pi^{\xi} \backslash N$ we have $E_{y}^{\xi} \subset F_{y}^{\xi}$ and $y \cdot \eta \notin N_{0}$.

Let us fix $y \in \Pi^{\xi} \backslash N$ and let $t \in J_{\hat{u}_{y}^{\xi}}$. Then $y=a+s \eta$ with $a \in \Pi^{\eta, \xi}$ and $s \in \mathbb{R} \backslash N_{0}$. Therefore

$$
J_{\hat{u}_{y}^{\xi}}=E_{y}^{\xi} \subset F_{a+s \eta}^{\xi}=\left(F_{s}\right)_{a}^{\xi}=\left(J_{u_{s}}\right)_{a}^{\xi}
$$

by (8.10), (8.12), and (8.14), so that $t \in\left(J_{u_{s}}\right)_{a}^{\xi}$, hence $a+t \xi \in J_{u_{s}}$. Let $x_{0}:=y+t \xi=$ $a+s \eta+t \xi$. Since $x_{0}+\Pi^{\eta}=s \eta+\Pi^{\eta}$ and $s \notin N_{2}$, we have $\mathcal{H}^{n-1}\left(S_{u} \cap\left(s \eta+\Pi^{\eta}\right)\right)=0$ and $u=\tilde{u} \mathcal{H}^{n-1}$-a.e. in $s \eta+\Pi^{\eta}$. Therefore the function $v$ considered in Theorem 7.1 satisfies $v(z)=u_{s}(z+a+t \xi)$ for $\mathcal{H}^{n-1}$-a.e. $z \in \Pi^{\eta}$. Since $a+t \xi \in J_{u_{s}}$, hypothesis (7.3) is satisfied with $b^{+} \neq b^{-}$. Therefore Theorem 7.1 implies that $x_{0} \in \Theta_{u}$. Since $x_{0}:=y+t \xi$ we have $t \in\left(\Theta_{u}\right)_{y}^{\xi}$. This proves (8.9) and concludes the proof of (8.8).

Let us prove that for $\mathcal{H}^{n-1}$-a.e. $y \in \Pi^{\xi}$ we have

$$
J_{\hat{u}_{y}^{\xi}} \subset\left(J_{u}^{\xi}\right)_{y}^{\xi} \text {. }
$$

By (8.3) and (8.8) for $\mathcal{H}^{n-1}$-a.e. $y \in \Pi^{\xi}$ and for every $t \in J_{\hat{u}_{y}^{\xi}}$ we have $y+t \xi \in J_{u}$ and

$$
\left(u^{+}(y+t \xi)-u^{-}(y+t \xi)\right) \cdot \xi=\left[\hat{u}_{y}^{\xi}\right](t) \neq 0,
$$

hence $y+t \xi \in J_{u}^{\xi}$ by (8.1). This proves (8.15) and concludes the proof of the theorem.

\section{Approximate symmetric differentiability}

In this section we prove that every $u \in \operatorname{GBD}(\Omega)$ has an approximate symmetric gradient $\mathcal{L}^{n}$-a.e. in $\Omega$. This means that for $\mathcal{L}^{n}$-a.e. $x \in \Omega$ there exists a symmetric matrix, denoted by $\mathcal{E} u(x)$, such that

$$
\operatorname{ap}_{y \rightarrow x} \frac{(u(y)-u(x)-\mathcal{E} u(x)(y-x)) \cdot(y-x)}{|y-x|^{2}}=0 .
$$

We also prove that the function $x \mapsto \mathcal{E} u(x)$, defined $\mathcal{L}^{n}$-a.e. in $\Omega$, belongs to $L^{1}\left(\Omega ; \mathbb{M}_{\text {sym }}^{n \times n}\right)$, where $\mathbb{M}_{\text {sym }}^{n \times n}$ is the space of symmetric $n \times n$ matrices. We also show that the one-dimensional slices of $\mathcal{E} u$ are related with the density $\nabla \hat{u}_{y}^{\xi}$ of the absolutely continuous part $D^{a} \hat{u}_{y}^{\xi}$ of the measure $D \hat{u}_{y}^{\xi}$ with respect to $\mathcal{L}^{1}$. 
Theorem 9.1. Let $u \in \operatorname{GBD}(\Omega)$. Then there exists a function $\mathcal{E} u \in L^{1}\left(\Omega ; \mathbb{M}_{\mathrm{sym}}^{n \times n}\right)$ such that (9.1) holds for $\mathcal{L}^{n}$-a.e. $x \in \Omega$. Moreover for every $\xi \in \mathbb{R}^{n} \backslash\{0\}$ and for $\mathcal{H}^{n-1}$-a.e. $y \in \Pi^{\xi}$ we have

$$
(\mathcal{E} u)_{y}^{\xi} \xi \cdot \xi=\nabla \hat{u}_{y}^{\xi} \quad \mathcal{L}^{1} \text {-a.e. on } \Omega_{y}^{\xi}
$$

Proof. Since the problem is local, we may assume that $u$ has compact support in $\Omega$. Let us fix $\xi \in \mathbb{R}^{n} \backslash\{0\}$. By modifying $u$ on a set of Lebesgue measure zero, we may assume that $u$ is a Borel function on $\Omega$ and $\hat{u}_{y}^{\xi} \in \mathrm{BV}_{\text {loc }}\left(\Omega_{y}^{\xi}\right)$ for every $y \in \Pi^{\xi}$. For every $x \in \Omega$ we define

$$
\begin{aligned}
\hat{u}^{\xi}(x) & :=\limsup _{\rho \rightarrow 0+} \frac{1}{2 \rho} \int_{-\rho}^{\rho} u(x+s \xi) \cdot \xi d s, \\
e^{\xi}(x) & :=\limsup _{\rho \rightarrow 0+} \frac{1}{\rho} \int_{0}^{\rho} \frac{\hat{u}^{\xi}(x+s \xi)-\hat{u}^{\xi}(x)}{s} d s .
\end{aligned}
$$

Then $u^{\xi}$ and $e^{\xi}$ are Borel functions and have compact support on $\Omega$. By an easy change of variables we can prove that

$$
e^{\rho \xi}(x)=\rho^{2} e^{\xi}(x) \quad \text { for every } \rho>0 \text { and every } x \in \Omega
$$

By the Lebesgue differentiation theorem for every $y \in \Pi^{\xi}$ we have

$$
\left(\hat{u}^{\xi}\right)_{y}^{\xi}=\hat{u}_{y}^{\xi} \quad \mathcal{L}^{1} \text {-a.e. in } \Omega_{y}^{\xi} .
$$

Since $\hat{u}_{y}^{\xi} \in \mathrm{BV}\left(\Omega_{y}^{\xi}\right)$ and $\left(\hat{u}^{\xi}\right)_{y}^{\xi}$ is a good representative of $\hat{u}_{y}^{\xi}$ by (9.3), using well known properties of BV functions in dimension one (see, e.g., [6, Section 3.2]) we deduce that

$$
\left(\nabla \hat{u}_{y}^{\xi}\right)(t)=\lim _{s \rightarrow 0} \frac{\left(\hat{u}^{\xi}\right)_{y}^{\xi}(t+s)-\left(\hat{u}^{\xi}\right)_{y}^{\xi}(t)}{s}=\left(e^{\xi}\right)_{y}^{\xi}(t)
$$

for every $y \in \Pi^{\xi}$ and for $\mathcal{L}^{1}$-a.e. $t \in \Omega_{y}^{\xi}$.

Let $g: \mathbb{R} \rightarrow[0,1)$ be an even continuous function, with $g(0)=0$, such that $g$ is strictly increasing and concave on $\mathbb{R}^{+}$. It is easy to prove that $g$ satisfies the triangle inequality

$$
g(s+t) \leq g(s)+g(t) \quad \text { for every } s, t \in \mathbb{R} .
$$

By (9.6) and (9.7) we have

$$
\lim _{\rho \rightarrow 0+} \frac{1}{\rho} \int_{0}^{\rho} g\left(\frac{\hat{u}_{y}^{\xi}(t+s)-\hat{u}_{y}^{\xi}(t)}{s}-\left(e^{\xi}\right)_{y}^{\xi}(t)\right) d s=0
$$

for every $y \in \Pi^{\xi}$ and for $\mathcal{L}^{1}$-a.e. $t \in \Omega_{y}^{\xi}$. By Fubini's theorem this implies that

$$
\lim _{\rho \rightarrow 0+} \frac{1}{\rho} \int_{0}^{\rho} g\left(\frac{u(x+s \xi) \cdot \xi-u(x) \cdot \xi}{s}-e^{\xi}(x)\right) d s=0
$$


for $\mathcal{L}^{n}$-a.e. $x \in \Omega$. Integrating over $\Omega$ and exchanging the order of integration we obtain

$$
\lim _{\rho \rightarrow 0+} \frac{1}{\rho} \int_{0}^{\rho}\left[\int_{\Omega} g\left(\frac{u(x+s \xi) \cdot \xi-u(x) \cdot \xi}{s}-e^{\xi}(x)\right) d x\right] d s=0
$$

for every $\xi \in \mathbb{R}^{n} \backslash\{0\}$. We define $e^{\xi}(x)=0$ for $\xi=0$. Note that (9.10) holds also in this case.

Let us fix $\eta \in \mathbb{R}^{n}$. By the triangle inequality (9.8) for every $s>0$ small enough we have

$$
\begin{aligned}
& \int_{\Omega} g\left(\frac{u(x+s \eta+s \xi) \cdot \xi-u(x+s \eta) \cdot \xi}{s}-e^{\xi}(x)\right) d x \\
& \leq \int_{\Omega} g\left(\frac{u(x+s \eta+s \xi) \cdot \xi-u(x+s \eta) \cdot \xi}{s}-e^{\xi}(x+s \eta)\right) d x \\
&+\int_{\Omega} g\left(e^{\xi}(x+s \eta)-e^{\xi}(x)\right) d x \\
&= \int_{\Omega} g\left(\frac{u(x+s \xi) \cdot \xi-u(x) \cdot \xi}{s}-e^{\xi}(x)\right) d x \\
&+\int_{\Omega} g\left(e^{\xi}(x+s \eta)-e^{\xi}(x)\right) d x
\end{aligned}
$$

where, in the last equality, we have used the fact that $u$ and $e^{\xi}$ have compact support in $\Omega$.

Let us prove that

$$
\lim _{s \rightarrow 0+} \int_{\Omega} g\left(e^{\xi}(x+s \eta)-e^{\xi}(x)\right) d x=0 .
$$

Let us fix $\tau \in \mathcal{T}$ with $\tau^{\prime}(t)>0$ for every $t \in \mathbb{R}$. By the continuity of translations in $L^{1}(\Omega)$ we have

$$
\lim _{s \rightarrow 0+} \int_{\Omega}\left|\tau\left(e^{\xi}(x+s \eta)\right)-\tau\left(e^{\xi}(x)\right)\right| d x=0 .
$$

This implies that for every sequence $s_{k} \rightarrow 0$ there exists a subsequence $s_{k_{j}}$ such that $\tau\left(e^{\xi}\left(x+s_{k_{j}} \eta\right)\right) \rightarrow \tau\left(e^{\xi}(x)\right)$ for $\mathcal{L}^{n}$-a.e. $x \in \Omega$. Since $\tau$ is invertible and the inverse function is continuous, we deduce that $e^{\xi}\left(x+s_{k_{j}} \eta\right) \rightarrow e^{\xi}(x)$ for $\mathcal{L}^{n}$-a.e. $x \in \Omega$. This implies that

$$
\lim _{j \rightarrow \infty} \int_{\Omega} g\left(e^{\xi}\left(x+s_{k_{j}} \eta\right)-e^{\xi}(x)\right) d x=0
$$

by the Dominated Convergence Theorem. Since the sequence $s_{k} \rightarrow 0$ is arbitrary, we obtain (9.12). That equality, together with (9.10) and (9.11), gives

$$
\lim _{\rho \rightarrow 0+} \frac{1}{\rho} \int_{0}^{\rho}\left[\int_{\Omega} g\left(\frac{u(x+s \eta+s \xi) \cdot \xi-u(x+s \eta) \cdot \xi}{s}-e^{\xi}(x)\right) d x\right] d s=0 .
$$

Let us fix $\xi, \eta \in \mathbb{R}^{n}$. We want to prove that the following parallelogram identity holds:

$$
e^{\xi+\eta}(x)+e^{\xi-\eta}(x)=2 e^{\xi}(x)+2 e^{\xi}(x) \quad \text { for } \mathcal{L}^{n} \text {-a.e. } x \in \Omega \text {. }
$$


By the parallelogram identity (7.1), the homogeneity condition (9.5), and the triangle inequality (9.10), for every $s>0$ small enough we have

$$
\begin{aligned}
& g\left(2 e^{\xi+\eta}(x)+2 e^{\xi-\eta}(x)-4 e^{\xi}(x)-4 e^{\xi}(x)\right) \\
& \leq g\left(e^{\xi+\eta}(x)-\frac{u(x+s \xi) \cdot(\xi+\eta)-u(x-s \eta) \cdot(\xi+\eta)}{s}\right) \\
&+g\left(e^{\xi+\eta}(x)-\frac{u(x+s \eta) \cdot(\xi+\eta)-u(x-s \xi) \cdot(\xi+\eta)}{s}\right) \\
&+g\left(e^{\xi-\eta}(x)-\frac{u(x+s \xi) \cdot(\xi-\eta)-u(x+s \eta) \cdot(\xi-\eta)}{s}\right) \\
&+g\left(e^{\xi-\eta}(x)-\frac{u(x-s \eta) \cdot(\xi-\eta)-u(x-s \xi) \cdot(\xi-\eta)}{s}\right) \\
&+g\left(e^{2 \xi}(x)-\frac{u(x+s \xi) \cdot(2 \xi)-u(x-s \xi) \cdot(2 \xi)}{s}\right) \\
&+g\left(e^{2 \eta}(x)-\frac{u(x+s \eta) \cdot(2 \eta)-u(x-s \eta) \cdot(2 \eta)}{s}\right) .
\end{aligned}
$$

Using (9.13) we obtain

$$
\lim _{\rho \rightarrow 0+} \frac{1}{\rho} \int_{0}^{\rho}\left[\int_{\Omega} g\left(2 e^{\xi+\eta}(x)+2 e^{\xi-\eta}(x)-4 e^{\xi}(x)-4 e^{\xi}(x)\right) d x\right] d s=0,
$$

which implies

$$
\int_{\Omega} g\left(2 e^{\xi+\eta}(x)+2 e^{\xi-\eta}(x)-4 e^{\xi}(x)-4 e^{\xi}(x)\right) d x=0 .
$$

Since $g(s)=0$ if and only if $s=0$, we obtain (9.14).

Let $\mathbb{Q}$ be the field of rational numbers. By (9.14) there exists a Borel set $N \subset \Omega$, with $\mathcal{L}^{n}(N)=0$, such that for every $x \in \Omega \backslash N$ the parallelogram identity

$$
e^{\xi+\eta}(x)+e^{\xi-\eta}(x)=2 e^{\xi}(x)+2 e^{\xi}(x)
$$

holds for every $\xi, \eta \in \mathbb{Q}^{n}$. Since $e^{\xi}(x)$ is also positively homogeneous of degree 2 by (9.5), arguing as in the proof of [15, Proposition 11.9] we deduce that for every $x \in \Omega \backslash N$ there exists a symmetric $\mathbb{Q}$-bilinear form $B_{x}: \mathbb{Q}^{n} \times \mathbb{Q}^{n} \rightarrow \mathbb{R}$ such that

$$
e^{\xi}(x)=B_{x}(\xi, \xi)
$$

for every $\xi \in \mathbb{Q}^{n}$. This implies that for every $x \in \Omega \backslash N$ there exists a symmetric matrix $\mathcal{E} u(x) \in \mathbb{M}_{\text {sym }}^{n \times n}$ such that

$$
e^{\xi}(x)=\mathcal{E} u(x) \xi \cdot \xi
$$

for every $\xi \in \mathbb{Q}^{n}$.

Let us fix $\xi_{0} \in \mathbb{R}^{n}$. We want to prove that (9.16) holds for $\xi=\xi_{0}$ and for $\mathcal{L}^{n}$-a.e. $x \in \Omega$. Let $\Xi$ be the vector subspace over $\mathbb{Q}$ generated by $\mathbb{Q}^{n} \cup\left\{\xi_{0}\right\}$. Since $\Xi$ is countable, 
there exists a Borel set $N_{0} \subset \mathbb{R}^{n}$, with $N_{0} \supset N$ and $\mathcal{L}^{n}\left(N_{0}\right)=0$, such that (9.15) holds for every $x \in \Omega \backslash N_{0}$ and for every $\xi, \eta \in \Xi$. Arguing as before we prove that for every $x \in \Omega \backslash N_{0}$ there exists a symmetric matrix $A(x) \in \mathbb{M}_{\text {sym }}^{n \times n}$ such that

$$
e^{\xi}(x)=A(x) \xi \cdot \xi
$$

for every $\xi \in \Xi$. Since $\mathbb{Q}^{n} \subset \Xi$ and $N \subset N_{0}$, equalities (9.16) and (9.17) hold for every $x \in \Omega \backslash N_{0}$ and for every $\xi \in \mathbb{Q}^{n}$. This implies that $A(x)=\mathcal{E} u(x)$ for every $x \in \Omega \backslash N_{0}$. Since (9.17) holds for every $x \in \Omega \backslash N_{0}$ and for every $\xi \in \Xi$, we deduce that the same is true for (9.16). Since $\xi_{0} \in \Xi$, we conclude that (9.16) holds for $\xi=\xi_{0}$ and for every $x \in \Omega \backslash N_{0}$.

Since $\xi_{0}$ is arbitrary, we have shown that for every $\xi \in \mathbb{R}^{n}$ we have

$$
e^{\xi}(x)=\mathcal{E} u(x) \xi \cdot \xi \quad \mathcal{L}^{n} \text {-a.e. in } \Omega .
$$

By Fubini's theorem, (9.18) gives

$$
\left(e^{\xi}\right)_{y}^{\xi}(t)=(\mathcal{E} u)_{y}^{\xi}(t) \xi \cdot \xi
$$

for every $\xi \in \mathbb{R}^{n} \backslash\{0\}$, for $\mathcal{H}^{n-1}$-a.e. $y \in \Pi^{\xi}$, and for $\mathcal{L}^{1}$-a.e. $t \in \Omega_{y}^{\xi}$. Together with (9.7), this property implies (9.2) for every $\xi \in \mathbb{R}^{n} \backslash\{0\}$ and for $\mathcal{H}^{n-1}$-a.e. $y \in \Pi^{\xi}$.

By (9.9) and (9.18) for every $\xi \in \mathbb{S}^{n-1}$ and for $\mathcal{L}^{n}$-a.e. $x \in \Omega$ we have

$$
\lim _{\rho \rightarrow 0+} \frac{1}{\rho} \int_{0}^{\rho} g\left(\frac{u(x+s \xi) \cdot(s \xi)-u(x) \cdot(s \xi)-\mathcal{E} u(x)(s \xi) \cdot(s \xi)}{s^{2}}\right) d s=0 .
$$

This implies

$$
\lim _{\rho \rightarrow 0+} \frac{1}{\rho^{n}} \int_{0}^{\rho} g\left(\frac{u(x+s \xi) \cdot(s \xi)-u(x) \cdot(s \xi)-\mathcal{E} u(x)(s \xi) \cdot(s \xi)}{s^{2}}\right) s^{n-1} d s=0 .
$$

Integrating over $\mathbb{S}^{n-1}$ and using the formula for polar coordinates we obtain

$$
\begin{aligned}
0 & =\lim _{\rho \rightarrow 0+} \frac{1}{\rho^{n}} \int_{B_{\rho}(0)} g\left(\frac{u(x+y) \cdot y-u(x) \cdot y-\mathcal{E} u(x) y \cdot y}{|y|^{2}}\right) d y= \\
& =\lim _{\rho \rightarrow 0+} \frac{1}{\rho^{n}} \int_{B_{\rho}(x)} g\left(\frac{(u(y)-u(x)-\mathcal{E} u(x)(y-x)) \cdot(y-x)}{|y-x|^{2}}\right) d y,
\end{aligned}
$$

which implies (9.1) by Chebyshev's inequality and by the properties of $g$.

It remains to prove that $\mathcal{E} u \in L^{1}\left(\Omega ; \mathbb{M}_{\text {sym }}^{n \times n}\right)$. Let $\hat{\mu}_{y}^{\xi}$ and $\hat{\mu}^{\xi}$ be the measures introduced in Definitions 4.8 and 4.10. By (9.2) we have

$$
\int_{\Omega_{y}^{\xi}}\left|(\mathcal{E} u)_{y}^{\xi} \xi \cdot \xi\right| d t=\left|D^{a} \hat{u}_{y}^{\xi}\right|\left(\Omega_{y}^{\xi}\right) \leq \hat{\mu}_{y}^{\xi}\left(\Omega_{y}^{\xi}\right)
$$


for every $\xi \in \mathbb{S}^{n-1}$ and for $\mathcal{H}^{n-1}$-a.e. $y \in \Pi^{\xi}$. Integrating over $\Pi^{\xi}$ and using Fubini's theorem and (4.6) for every $\xi \in \mathbb{S}^{n-1}$ we obtain

$$
\int_{\Omega}|\mathcal{E} u \xi \cdot \xi| d x \leq \hat{\mu}^{\xi}(\Omega) \leq \hat{\mu}_{u}(\Omega)<\infty,
$$

where $\hat{\mu}_{u}$ is the measure introduced in Definition 4.16. This implies that $\mathcal{E} u \in$ $L^{1}\left(\Omega ; \mathbb{M}_{\text {sym }}^{n \times n}\right)$ and concludes the proof of the theorem.

Remark 9.2. By the structure theorem for BD functions (see [5, Theorem 4.5]) and by Theorem 9.1, for every $u \in \operatorname{BD}(\Omega)$ the function $\mathcal{E} u$ coincides with the density of the absolutely continuous part of $E u$ with respect to $\mathcal{L}^{n}$.

Remark 9.3. Let $\sigma: \mathbb{R} \rightarrow \mathbb{R}$ be the truncation function defined by $\sigma(s):=\min \{|s|, 1\}$. By Theorems 8.1 and 9.1 for every $u \in \operatorname{GBD}(\Omega)$ we have

$$
\left|D^{a} \hat{u}_{y}^{\xi}\right|\left(B_{y}^{\xi}\right)+\int_{B_{y}^{\xi} \cap J_{\hat{u} y}^{\xi}} \sigma\left(\left[\hat{u}_{y}^{\xi}\right]\right) d \mathcal{H}^{0}=\int_{B_{y}^{\xi}}\left|(\mathcal{E} u)_{y}^{\xi} \xi \cdot \xi\right| d t+\int_{B_{y}^{\xi} \cap\left(J_{u}^{\xi}\right)_{y}^{\xi}} \sigma\left([u]_{y}^{\xi} \cdot \xi\right) d \mathcal{H}^{0}
$$

for every $\xi \in \mathbb{S}^{n-1}$, for $\mathcal{H}^{n-1}$-a.e. $y \in \Pi^{\xi}$, and for every Borel set $B \subset \Omega$. By the area formula (see, e.g., [6, Theorem 2.71]) and by Fubini's theorem it follows that for every $u \in \operatorname{GSBD}(\Omega)$ the measure $\hat{\mu}_{u}^{\xi}$ defined by (4.5) satisfies

$\hat{\mu}_{u}^{\xi}(B)=\int_{B}|\mathcal{E} u \xi \cdot \xi| d x+\int_{B \cap J_{u}} \sigma([u] \cdot \xi)\left|v_{u} \cdot \xi\right| d \mathcal{H}^{n-1} \leq \int_{B}|\mathcal{E} u| d x+\mathcal{H}^{n-1}\left(B \cap J_{u}\right)$

for every $\xi \in \mathbb{S}^{n-1}$ and for every Borel set $B \subset \Omega$. Therefore for every $u \in \operatorname{GSBD}(\Omega)$ the measure $\hat{\mu}_{u}$ introduced in Definition 4.16 satisfies the estimate

$$
\hat{\mu}_{u}(B) \leq \int_{B}|\mathcal{E} u| d x+\mathcal{H}^{n-1}\left(B \cap J_{u}\right) \quad \text { for every Borel set } B \subset \Omega .
$$

\section{Compactness and slicing}

In this section we prove some extensions of the well-known Fréchet-Kolmogorov compactness criterion in $L^{1}$. In particular we are interested in some conditions that imply sequential compactness with respect to $\mathcal{L}^{n}$-a.e. pointwise convergence. The main result is obtained by assuming suitable properties of the one-dimensional slices.

To simplify the exposition, in this section every function $u$ defined on $\Omega$ is always extended to $\mathbb{R}^{n}$ by setting $u(x)=0$ for every $x \in \mathbb{R}^{n} \backslash \Omega$. These results are based on the notion of modulus of continuity, made precise by the following definition.

Definition 10.1. A modulus of continuity is an increasing continuous function $\omega$ : $\mathbb{R}^{+} \rightarrow \mathbb{R}^{+}$such that $\omega(0)=0$.

The first lemma provides a compactness result with respect to pointwise $\mathcal{L}^{n}$-a.e. convergence. Note that the usual $L^{1}$ bound is replaced by (10.3). 
Lemma 10.2. Let $\mathcal{U}$ be a set of $\mathcal{L}^{n}$-measurable functions from $\Omega$ into $\mathbb{R}^{n}$, let $g: \mathbb{R}^{+} \rightarrow \mathbb{R}^{+}$be a nondecreasing continuous function satisfying

$$
g(0)=0 \text { and } \liminf _{s \rightarrow 0+} \frac{g(s)}{s}>0,
$$

and let $\psi_{0}: \mathbb{R}^{+} \rightarrow \mathbb{R}^{+}$be an increasing continuous function with

$$
\lim _{s \rightarrow \infty} \psi_{0}(s)=\infty .
$$

Assume that there exist a constant $M \in \mathbb{R}^{+}$and a modulus of continuity $\omega$ such that

$$
\begin{gathered}
\int_{\Omega} \psi_{0}(|u|) d x \leq M, \\
\int_{\mathbb{R}^{n}} g(|u(x+h \xi)-u(x)|) d x \leq \omega(h)
\end{gathered}
$$

for every $u \in \mathcal{U}$, every $\xi \in \mathbb{S}^{n-1}$, and every $0<h<1$. Then every sequence in $\mathcal{U}$ has a subsequence that converges pointwise $\mathcal{L}^{n}$-a.e. on $\Omega$ to an $\mathcal{L}^{n}$-measurable function $u: \Omega \rightarrow \mathbb{R}^{n}$.

Proof. By (10.1) there exist $a, r>0$ such that $a s \leq g(s)$ for every $0 \leq s \leq 2 r$. Let $\varphi: \mathbb{R}^{n} \rightarrow B_{r}(0)$ be the homeomorphism defined by

$$
\varphi(z)=\frac{r z}{\sqrt{r^{2}+|z|^{2}}} .
$$

There exists $c \in \mathbb{R}^{+}$such that $\left|\varphi\left(z_{2}\right)-\varphi\left(z_{1}\right)\right| \leq c\left|z_{2}-z_{1}\right|$ for every $z_{1}, z_{2} \in \mathbb{R}^{n}$. Therefore

$$
\begin{aligned}
\frac{a}{c} \int_{\mathbb{R}^{n}}|\varphi(u(x+h \xi))-\varphi(u(x))| d x & \leq \int_{\mathbb{R}^{n}} g(|\varphi(u(x+h \xi))-\varphi(u(x))|) d x \\
& \leq \int_{\mathbb{R}^{n}} g(c|u(x+h \xi)-u(x)|) d x \leq \omega(c h)
\end{aligned}
$$

for every $u \in \mathcal{U}$, every $\xi \in \mathbb{S}^{n-1}$, and every $0<h<1$. By the Fréchet-Kolmogorov compactness criterion every sequence $u_{k}$ in $\mathcal{U}$ has a subsequence, not relabelled, such that $v_{k}:=\varphi\left(u_{k}\right)$ converges strongly in $L^{1}\left(\Omega ; \mathbb{R}^{n}\right)$ to a function $v: \Omega \rightarrow \bar{B}_{r}(0)$. Passing to a further subsequence we may assume that $v_{k}$ converges to $v$ pointwise $\mathcal{L}^{n}$-a.e. on $\Omega$.

Let us prove that $|v(x)|<r$ for $\mathcal{L}^{n}$-a.e. $x \in \Omega$. Let $A:=\{x \in \Omega:|v(x)|=r\}$. By (10.5) we have

$$
u_{k}=\frac{r v_{k}}{\sqrt{r^{2}-v_{k}^{2}}},
$$

so that $\left|u_{k}(x)\right| \rightarrow \infty$ for $\mathcal{L}^{n}$-a.e. $x \in A$. By (10.2) this implies $\psi_{0}\left(\left|u_{k}(x)\right|\right) \rightarrow \infty$ for $\mathcal{L}^{n}$-a.e. $x \in A$. By (10.3) and by Fatou's lemma we conclude that $\mathcal{L}^{n}(A)=0$, hence $|v(x)|<r$ for $\mathcal{L}^{n}$-a.e. $x \in \Omega$. By (10.6) we deduce that $u_{k}$ converges pointwise $\mathcal{L}^{n}$-a.e. on $\Omega$ to the function

This concludes the proof.

$$
u=\frac{r v}{\sqrt{r^{2}-v^{2}}} .
$$


The next lemma shows that in the Fréchet-Kolmogorov condition it is enough to consider only the components of a vector function along the translation vectors.

Lemma 10.3. Let $\mathcal{U}$ be a set of $\mathcal{L}^{n}$-measurable functions from $\Omega$ into $\mathbb{R}^{n}$, let $g: \mathbb{R}^{+} \rightarrow \mathbb{R}^{+}$be a nondecreasing continuous function such that

$$
g(0)=0 \quad \text { and } \quad g(s+t) \leq g(s)+g(t)
$$

for every $s, t \in \mathbb{R}^{+}$, and let $\psi_{0}: \mathbb{R}^{+} \rightarrow \mathbb{R}^{+}$be an increasing continuous function satisfying (10.2). Assume that there exist a constant $M \in \mathbb{R}^{+}$and two moduli of continuity $\omega$ and $\hat{\omega}$ such that (10.3) holds and

$$
\begin{gathered}
\int_{\mathbb{R}^{n}} g(|u(x+h \xi) \cdot \xi-u(x) \cdot \xi|) d x \leq \omega(h), \\
g(h s) \leq \hat{\omega}(h) \psi_{0}(s)
\end{gathered}
$$

for every $u \in \mathcal{U}$, every $\xi \in \mathbb{S}^{n-1}$, every $0<h<1$, and every $s \in \mathbb{R}^{+}$. Then there exists a modulus of continuity $\tilde{\omega}$ such that

$$
\int_{\mathbb{R}^{n}} g(|u(x+h \xi)-u(x)|) d x \leq \tilde{\omega}(h)
$$

for every $u \in \mathcal{U}$, every $\xi \in \mathbb{S}^{n-1}$, and every $0<h<1$.

Proof. Let us fix $\xi \in \mathbb{S}^{n-1}$ and $0<h<1 / 2$. There exist $\eta^{1}, \ldots, \eta^{n-1} \in \mathbb{S}^{n-1}$ such that $\xi, \eta^{1}, \ldots, \eta^{n-1}$ form an orthonormal basis. Then

$$
|u(x+h \xi)-u(x)| \leq|u(x+h \xi) \cdot \xi-u(x) \cdot \xi|+\sum_{i=1}^{n-1}\left|u(x+h \xi) \cdot \eta^{i}-u(x) \cdot \eta^{i}\right|
$$

for every $x \in \mathbb{R}^{n}$. By the triangle inequality we have

$$
\begin{aligned}
& \left|u(x+h \xi) \cdot \eta^{i}-u(x) \cdot \eta^{i}\right| \\
& \leq\left|u(x+h \xi) \cdot \eta^{i}-u\left(x+\sqrt{h} \eta^{i}\right) \cdot \eta^{i}\right|+\left|u\left(x+\sqrt{h} \eta^{i}\right) \cdot \eta^{i}-u(x) \cdot \eta^{i}\right| .
\end{aligned}
$$

Let

so that

$$
\eta_{h}^{i}:=\frac{\sqrt{h}}{\sqrt{h+h^{2}}} \eta^{i}-\frac{h}{\sqrt{h+h^{2}}} \xi
$$

$$
\left|\eta_{h}^{i}\right|=1, \quad h \xi-\sqrt{h} \eta^{i}=-s_{h} \eta_{h}^{i}, \quad\left|\eta_{h}^{i}-\eta^{i}\right| \leq \sqrt{2} \sqrt{h},
$$

with $s_{h}:=\sqrt{h+h^{2}}$. By the triangle inequality and (10.13) we have

$$
\begin{aligned}
\mid u(x+h \xi) & \cdot \eta^{i}-u\left(x+\sqrt{h} \eta^{i}\right) \cdot \eta^{i} \mid \\
\leq & |u(x+h \xi)|\left|\eta^{i}-\eta_{h}^{i}\right|+\left|u(x+h \xi) \cdot \eta_{h}^{i}-u\left(x+\sqrt{h} \eta^{i}\right) \cdot \eta_{h}^{i}\right| \\
& +\left|u\left(x+\sqrt{h} \eta_{h}^{i}\right)\right|\left|\eta_{h}^{i}-\eta^{i}\right| \\
\leq & \left|u\left(x+\sqrt{h} \eta^{i}-s_{h} \eta_{h}^{i}\right) \cdot \eta_{h}^{i}-u\left(x+\sqrt{h} \eta^{i}\right) \cdot \eta_{h}^{i}\right| \\
& +|u(x+h \xi)| \sqrt{2} \sqrt{h}+\left|u\left(x+\sqrt{h} \eta_{h}^{i}\right)\right| \sqrt{2} \sqrt{h} .
\end{aligned}
$$


From (10.7), (10.8), (10.11), (10.12), and (10.14) for every $u \in \mathcal{U}$ we obtain

$$
\begin{aligned}
& \int_{\mathbb{R}^{n}} g(|u(x+h \xi)-u(x)|) d x \\
& \leq \int_{\mathbb{R}^{n}} g(|u(x+h \xi) \cdot \xi-u(x) \cdot \xi|) d x+\sum_{i=1}^{n-1} \int_{\mathbb{R}^{n}} g\left(\left|u\left(x-s_{h} \eta_{h}^{i}\right) \cdot \eta_{h}^{i}-u(x) \cdot \eta_{h}^{i}\right|\right) d x \\
& \quad+2(n-1) \int_{\mathbb{R}^{n}} g(|u(x)| \sqrt{2} \sqrt{h}) d x+\sum_{i=1}^{n-1} \int_{\mathbb{R}^{n}} g\left(\left|u\left(x+\sqrt{h} \eta^{i}\right) \cdot \eta^{i}-u(x) \cdot \eta^{i}\right|\right) d x \\
& \leq \omega(h)+(n-1) \omega\left(\sqrt{h+h^{2}}\right)+2(n-1) \int_{\mathbb{R}^{n}} g(|u(x)| \sqrt{2} \sqrt{h}) d x+(n-1) \omega(\sqrt{h}) .
\end{aligned}
$$

By (10.3) and (10.9) we have

$$
\int_{\mathbb{R}^{n}} g(|u(x)| \sqrt{2} \sqrt{h}) d x \leq \hat{\omega}(\sqrt{2} \sqrt{h}) \int_{\mathbb{R}^{n}} \psi_{0}(|u(x)|) d x \leq M \hat{\omega}(\sqrt{2} \sqrt{h}),
$$

which, together with the previous inequality, gives

$$
\begin{aligned}
\int_{\mathbb{R}^{n}} g(|u(x+h \xi)-u(x)|) d x \\
\quad \leq \omega(h)+(n-1) \omega\left(\sqrt{h+h^{2}}\right)+2(n-1) M \hat{\omega}(\sqrt{2} \sqrt{h})+(n-1) \omega(\sqrt{h}) .
\end{aligned}
$$

for every $0<h<1 / 2$. By the triangle inequality (10.7) this implies that (10.10) holds for every $0<h<1$ with $\tilde{\omega}(h):=2 \omega(h / 2)+2(n-1) \omega\left(\sqrt{2 h+h^{2}} / 2\right)+4(n-1) M \hat{\omega}(\sqrt{h})+$ $2(n-1) \omega(\sqrt{h} / \sqrt{2})$.

Remark 10.4. Inequality (10.9) is satisfied if

$$
g(s) \leq \psi_{0}(s) \quad \text { and } \quad \psi_{0}(h s) \leq \hat{\omega}(h) \psi_{0}(s)
$$

for every $s \in \mathbb{R}^{+}$and every $0<h<1$. Note that the second inequality in (10.15) holds when $\psi_{0}(s):=s^{p}$ with $p>0$. In particular, Lemma 10.3 can be applied with $g(s)=\psi_{0}(s)=s$ for every $s \in \mathbb{R}^{+}$.

Remark 10.5. Inequality (10.9) is satisfied if

$$
\psi_{0}(0)>0 \text { and } \lim _{s \rightarrow \infty} \frac{\psi_{0}(s)}{g(s)}=\infty .
$$

Indeed, (10.9) is equivalent to

$$
\lim _{h \rightarrow 0+} \sup _{s \in \mathbb{R}^{+}} \frac{g(h s)}{\psi_{0}(s)}=0 .
$$

By (10.16) the supremum is attained at a point $s_{h}$. Let $h_{j}$ be a sequence in $\mathbb{R}^{+}$converging to 0 . If $s_{h_{j}}$ is bounded, then $g\left(h_{j} s_{h_{j}}\right) / \psi_{0}\left(s_{h_{j}}\right) \leq g\left(h_{j} s_{h_{j}}\right) / \psi_{0}(0) \rightarrow 0$ since $g$ is continuous and $g(0)=0$ by (10.7). If $s_{h_{j}} \rightarrow \infty$, then $g\left(h_{j} s s_{h_{j}}\right) / \psi_{0}\left(s_{h_{j}}\right) \leq g\left(s_{h_{j}}\right) / \psi_{0}\left(s_{h_{j}}\right) \rightarrow 0$ 
by (10.16). Since every sequence $s_{h_{j}}$ has either a bounded subsequence or a subsequence diverging to $\infty$, we obtain (10.17). Since (10.7) gives $g(s) \leq 2 s g(1)$ for every $s \geq 1$, (10.16) is always satisfied if $\psi_{0}(0)>0$ and $\psi_{0}(s) / s \rightarrow \infty$ as $s \rightarrow \infty$.

Remark 10.6. When $\Omega$ has a Lipschitz boundary, Lemma 10.3 provides a quick proof of the compactness of the embedding of $\operatorname{BD}(\Omega)$ into $L^{1}\left(\Omega ; \mathbb{R}^{n}\right)$. For every $u \in C_{c}^{\infty}\left(\mathbb{R}^{n} ; \mathbb{R}^{n}\right)$, every $\xi \in \mathbb{S}^{n-1}$, and every $h>0$ we have

$$
|u(x+h \xi) \cdot \xi-u(x) \cdot \xi| \leq \int_{0}^{h}|D u(x+t \xi) \xi \cdot \xi| d t=\int_{0}^{h}|E u(x+t \xi) \xi \cdot \xi| d t
$$

where $(E u)_{i j}=\frac{1}{2}\left(D_{i} u_{j}+D_{j} u_{i}\right)$. It follows that

$$
\int_{\mathbb{R}^{n}}|u(x+h \xi) \cdot \xi-u(x) \cdot \xi| d x \leq \int_{0}^{h}\left(\int_{\mathbb{R}^{n}}|E u(x+t \xi) \xi \cdot \xi| d x\right) d t \leq h \int_{\mathbb{R}^{n}}|E u(x)| d x .
$$

If $u \in \mathrm{BD}(\Omega)$, we can approximate by convolutions its extension, which belongs to $\mathrm{BD}\left(\mathbb{R}^{n}\right)$ by the regularity of the boundary, and we get

$$
\int_{\mathbb{R}^{n}}|u(x+h \xi) \cdot \xi-u(x) \cdot \xi| d x \leq h|E u|\left(\mathbb{R}^{n}\right) .
$$

If $\mathcal{U}$ is a bounded subset of $\operatorname{BD}(\Omega)$, we can apply Lemma 10.3 with $g(s)=\psi_{0}(s)=s$ (see Remark 10.4) and we find that there exists a modulus of continuity $\tilde{\omega}$ such that

$$
\int_{\mathbb{R}^{n}}|u(x+h \xi)-u(x)| d x \leq \tilde{\omega}(h)
$$

for every $u \in \mathcal{U}$ and every $0<h<1$. By the Fréchet-Kolmogorov compactness criterion $\mathcal{U}$ is relatively compact in $L^{1}\left(\Omega ; \mathbb{R}^{n}\right)$.

In the next lemma we obtain the relative compactness with respect to pointwise $\mathcal{L}^{n}$ a.e. convergence from the behaviour of the one-dimensional slices. The proof follows the lines of [1, Theorem 6.6]. The main difference is that our assumptions concern only the components $u \cdot \xi$ of $u$ and the corresponding slices in the same direction $\xi$. Moreover we cannot assume $L^{\infty}$ bounds in view of the application to Theorem 11.1. This makes the statement of the lemma quite involved.

Lemma 10.7. Let $\mathcal{U}$ be a set of $\mathcal{L}^{n}$-measurable functions from $\Omega$ into $\mathbb{R}^{n}$, let $g: \mathbb{R}^{+} \rightarrow$ $\mathbb{R}^{+}$be a nondecreasing continuous function satisfying (10.1), (10.7), and

$$
g(s) \leq s \quad \text { for every } s \in \mathbb{R}^{+},
$$

and let $\psi_{0}: \mathbb{R}^{+} \rightarrow \mathbb{R}^{+}$be an increasing continuous function satisfying (10.2). Assume that there exist $M \in \mathbb{R}^{+}$such that (10.3) holds for every $u \in \mathcal{U}$ and a modulus of continuity $\hat{\omega}$ such that (10.9) holds for every $0<h<1$ and every $s \in \mathbb{R}^{+}$. Assume also that for every $\delta>0$ we can find a modulus of continuity $\omega_{\delta}$ such that for every $\xi \in \mathbb{S}^{n-1}$ there exists a set $\mathcal{V}_{\delta}^{\xi}$ of $\mathcal{L}^{n}$-measurable functions from $\Omega$ into $\mathbb{R}$ with the following properties: 
(a) for every $u \in \mathcal{U}$ there exists $v \in \mathcal{V}_{\delta}^{\xi}$ with

$$
\int_{\mathbb{R}^{n}} g(|u(x) \cdot \xi-v(x)|) d x \leq \delta
$$

(b) for every $v \in \mathcal{V}_{\delta}^{\xi}$ and for $\mathcal{H}^{n-1}$-a.e. $y \in \Pi^{\xi}$ we have

$$
\int_{\mathbb{R}}\left|v_{y}^{\xi}(t+h)-v_{y}^{\xi}(t)\right| d t \leq \omega_{\delta}(h) \quad \text { for every } 0<h<1 .
$$

Then every sequence in $\mathcal{U}$ has a subsequence that converges pointwise $\mathcal{L}^{n}$-a.e. on $\Omega$ to an $\mathcal{L}^{n}$-measurable function $u: \Omega \rightarrow \mathbb{R}^{n}$. If, in addition, $g(s)=s$ for every $s \in \mathbb{R}^{+}$, then $\mathcal{U} \subset L^{1}\left(\Omega ; \mathbb{R}^{n}\right)$ and every sequence in $\mathcal{U}$ has a subsequence that converges strongly in $L^{1}\left(\Omega ; \mathbb{R}^{n}\right)$.

Note that the modulus of continuity in (10.20) does not depend on $y$ or $\xi$.

Proof of Lemma 10.7. Let us fix $u \in \mathcal{U}, \delta>0$, and $\xi \in \mathbb{S}^{n-1}$. Then there exists $v \in \mathcal{V}_{\delta}^{\xi}$ satisfying (10.19). By (10.7), (10.18), and (10.20), for every $0<h<1$ we have

$$
\begin{aligned}
\int_{\mathbb{R}^{n}} g(|u(x+h \xi) \cdot \xi-u(x) \cdot \xi|) d x \leq 2 \delta+\int_{\mathbb{R}^{n}}|v(x+h \xi)-v(x)| d x \\
=2 \delta+\int_{\pi^{\xi}(\Omega)}\left(\int_{\mathbb{R}}\left|v_{y}^{\xi}(t+h)-v_{y}^{\xi}(t)\right| d t\right) d \mathcal{H}^{n-1}(y) \leq 2 \delta+c_{\Omega} \omega_{\delta}(h),
\end{aligned}
$$

where $c_{\Omega}:=\omega_{n-1} \operatorname{diam}(\Omega)^{n-1}$. Let

$$
\omega(h):=\inf _{\delta>0}\left(2 \delta+c_{\Omega} \omega_{\delta}(h)\right) .
$$

By (10.21) we have (10.8) for every $u \in \mathcal{U}$, every $\xi \in \mathbb{S}^{n-1}$, and every $0<h<1$. Since $\omega(h) \rightarrow 0$ as $h \rightarrow 0+$, by Lemma 10.3 there exists a modulus of continuity $\tilde{\omega}$ such that (10.10) holds. The main conclusion follows now from Lemma 10.2.

If $g(s)=s$, then $s \leq \hat{\omega}(1) \psi_{0}(s)$ for every $s \in \mathbb{R}^{+}$by (10.9). Therefore (10.3) implies that $\mathcal{U} \subset L^{1}\left(\Omega ; \mathbb{R}^{n}\right)$ and $\mathcal{U}$ is bounded in $L^{1}\left(\Omega ; \mathbb{R}^{n}\right)$. The relative compactness in $L^{1}\left(\Omega ; \mathbb{R}^{n}\right)$ follows now from the Fréchet-Kolmogorov criterion.

In the proof of the compactness theorem for $\operatorname{GBD}(\Omega)$ we need the following estimate of the modulus of continuity in $L^{1}$ of the translations of $\mathrm{BV}$ functions of one real variable.

Lemma 10.8. Let $z \in \mathrm{BV}(\mathbb{R})$. Assume that there exist constants $a, b>0$ such that

$$
|D z|\left(\mathbb{R} \backslash J_{z}^{1}\right)+\mathcal{H}^{0}\left(J_{z}^{1}\right) \leq a \text { and }\|z\|_{L^{\infty}(\mathbb{R})} \leq b .
$$

Then

$$
\int_{\mathbb{R}}|z(t+h)-z(t)| d t \leq(a+2 a b) h \quad \text { for every } h>0 .
$$


Proof. By (10.22), $\mathcal{H}^{0}\left(J_{z}^{1}\right) \leq a<\infty$ and for every $t \in J_{z}^{1}$ we have $|D z|(\{t\})=|[z](t)|$ $\leq 2 b$, so that $|D z|\left(J_{z}^{1}\right) \leq 2 a b$. Using (10.22) again, we obtain $|D z|(\mathbb{R}) \leq a+2 a b$. Regularizing $z$ by convolutions, we find a sequence $z_{k}$ in $C^{\infty}(\mathbb{R}) \cap \mathrm{BV}(\mathbb{R})$ such that

$$
z_{k} \rightarrow z \quad \text { strongly in } L^{1}(\mathbb{R}) \quad \text { and } \quad \int_{\mathbb{R}}\left|z_{k}^{\prime}\right| d t \leq a+2 a b \quad \text { for every } k .
$$

For every $t \in \mathbb{R}$ and every $h>0$ we have

$$
\left|z_{k}(t+h)-z_{k}(t)\right| \leq \int_{0}^{h}\left|z_{k}^{\prime}(t+s)\right| d s .
$$

By (10.24), integrating over $\mathbb{R}$ and interchanging the order of integration we get

$$
\begin{aligned}
& \int_{\mathbb{R}}\left|z_{k}(t+h)-z_{k}(t)\right| d t \leq \int_{\mathbb{R}}\left(\int_{0}^{h}\left|z_{k}^{\prime}(t+s)\right| d s\right) d t \\
&=\int_{0}^{h}\left(\int_{\mathbb{R}}\left|z_{k}^{\prime}(t+s)\right| d t\right) d s=h \int_{\mathbb{R}}\left|z_{k}^{\prime}(t)\right| d t \leq(a+2 a b) h .
\end{aligned}
$$

Passing to the limit as $k \rightarrow \infty$ and using (10.24) we obtain (10.23).

\section{Two compactness results}

In this section we prove the following analogue of the compact embedding of $\operatorname{BD}(\Omega)$ into $L^{1}\left(\Omega ; \mathbb{R}^{n}\right)$ : every subset of $\operatorname{GBD}(\Omega)$ satisfying uniform bounds for the measures $\hat{\mu}_{u}^{\xi}$ and for suitable integrals involving $u$ has a subsequence that converges pointwise $\mathcal{L}^{n}$ a.e. on $\Omega$. This allows us to obtain a compactness result for $\operatorname{GSBD}(\Omega)$, following the proof of the analogous result for $\operatorname{SBD}(\Omega)$ developed in [9]. As in the previous section, every function $u$ defined on $\Omega$ is always extended to $\mathbb{R}^{n}$ by setting $u(x)=0$ for every $x \in \mathbb{R}^{n} \backslash \Omega$.

Theorem 11.1. Let $\mathcal{U}$ be a subset of $\operatorname{GBD}(\Omega)$. Suppose that there exist a constant $M \in$ $\mathbb{R}^{+}$and an increasing continuous function $\psi_{0}: \mathbb{R}^{+} \rightarrow \mathbb{R}^{+}$, with

$$
\lim _{s \rightarrow \infty} \psi_{0}(s)=\infty
$$

such that for every $u \in \mathcal{U}$ and for every $\xi \in \mathbb{S}^{n-1}$ we have

$$
\int_{\Omega} \psi_{0}(|u|) d x \leq M \quad \text { and } \quad \hat{\mu}_{u}^{\xi}(\Omega) \leq M
$$

where $\hat{\mu}_{u}^{\xi}$ is the measure introduced in Definition 4.10. Then every sequence in $\mathcal{U}$ has a subsequence that converges pointwise $\mathcal{L}^{n}$-a.e. on $\Omega$ to an $\mathcal{L}^{n}$-measurable function $u: \Omega \rightarrow \mathbb{R}^{n}$. If, in addition

$$
\lim _{s \rightarrow \infty} \frac{\psi_{0}(s)}{s}=\infty
$$

then $\mathcal{U} \subset L^{1}\left(\Omega ; \mathbb{R}^{n}\right)$ and every sequence in $\mathcal{U}$ has a subsequence that converges strongly in $L^{1}\left(\Omega ; \mathbb{R}^{n}\right)$. 
Proof. It is enough to prove the result for every relatively compact open subset of $\Omega$. Therefore it is not restrictive to assume that $\Omega$ is the union of a finite number of open rectangles. This implies, in particular, that $\mathcal{H}^{0}\left(\partial\left(\Omega_{y}^{\xi}\right)\right)<\infty$ for every $\xi \in \mathbb{S}^{n-1}$ and every $y \in \Pi^{\xi}$, so that for every $u \in \mathcal{U}$ the slice $\hat{u}_{y}^{\xi}$ belongs to $\mathrm{BV}\left(\Omega_{y}^{\xi}\right)$ (see Proposition 3.4), and hence to $\mathrm{BV}(\mathbb{R})$.

To prove the main assertion, it is enough to show that $\mathcal{U}$ satisfies the hypotheses of Lemma 10.7. For every $u \in \mathcal{U}, \xi \in \mathbb{S}^{n-1}$, and $a>0$ we define

$$
\hat{A}_{u}^{\xi, a}:=\left\{y \in \Pi^{\xi}: \hat{u}_{y}^{\xi} \in \mathrm{BV}(\mathbb{R}),\left|D \hat{u}_{y}^{\xi}\right|\left(\mathbb{R} \backslash J_{\hat{u}_{y}^{\xi}}^{1}\right)+\mathcal{H}^{0}\left(J_{\hat{u}_{y}^{\xi}}^{1}\right) \leq a\right\} .
$$

Moreover we set $\hat{B}_{u}^{\xi, a}:=\Pi^{\xi} \backslash \hat{A}_{u}^{\xi, a}$ and we define

$$
A_{u}^{\xi, a}:=\left\{x \in \Omega: \pi^{\xi}(x) \in \hat{A}_{u}^{\xi, a}\right\} \quad \text { and } \quad B_{u}^{\xi, a}:=\left\{x \in \Omega: \pi^{\xi}(x) \in \hat{B}_{u}^{\xi, a}\right\} .
$$

Since for $\mathcal{H}^{n-1}$-a.e. $y \in \hat{B}_{u}^{\xi, a}$ we have $\hat{u}_{y}^{\xi} \in \mathrm{BV}(\mathbb{R})$ and

$$
\left|D \hat{u}_{y}^{\xi}\right|\left(\Omega_{y}^{\xi} \backslash J_{\hat{u}_{y}^{\xi}}^{1}\right)+\mathcal{H}^{0}\left(\Omega_{y}^{\xi} \cap J_{\hat{u}_{y}^{\xi}}^{1}\right)+\mathcal{H}^{0}\left(\partial\left(\Omega_{y}^{\xi}\right)\right)>a,
$$

by Chebyshev's inequality and by (4.4), (4.5), and (11.2) we have

$$
\mathcal{H}^{n-1}\left(\hat{B}_{u}^{\xi, a}\right) \leq \frac{M+\mathcal{H}^{n-1}(\partial \Omega)}{a},
$$

hence by Fubini's theorem

$$
\mathcal{L}^{n}\left(B_{u}^{\xi, a}\right) \leq \frac{M+\mathcal{H}^{n-1}(\partial \Omega)}{a} \operatorname{diam}(\Omega) .
$$

For every $b>0$ let $\sigma_{b}$ be the truncation function defined by $\sigma_{b}(t)=-b$ for $t \leq-b$, $\sigma_{b}(t)=t$ for $-b \leq t \leq b$, and $\sigma_{b}(t)=b$ for $t \geq b$. We define $v_{u, b}^{\xi, a} \in L^{1}(\Omega)$ by setting

$$
v_{u, b}^{\xi, a}:= \begin{cases}\sigma_{b}(u \cdot \xi) & \text { in } A_{u}^{\xi, a}, \\ 0 & \text { in } B_{u}^{\xi, a} .\end{cases}
$$

Let $g$ be a function satisfying all assumptions of Lemma 10.7 and such that

$$
\lim _{s \rightarrow \infty} \frac{\psi_{0}(s)}{g(s)}=\infty
$$

For every $\delta>0$ there exists $b_{\delta}>0$ such that $g(s) \leq \frac{\delta}{4 M} \psi_{0}(s)$ for every $s \geq b_{\delta}$. By (11.6) there exists $a_{\delta}>0$ such that $g(b) \mathcal{L}^{n}\left(B_{u}^{\xi, a}\right) \leq \delta / 2$. Therefore (11.2) gives

$$
\begin{aligned}
\int_{\Omega} g\left(\left|u \cdot \xi-v_{u, b_{\delta}}^{\xi, a}\right|\right) d x & =\int_{A_{u}^{\xi, a}} g\left(\left|u \cdot \xi-\sigma_{b_{\delta}}(u \cdot \xi)\right|\right) d x+\int_{B_{u}^{\xi, a}} g(|u \cdot \xi|) d x \\
& \leq 2 \int_{\{|u \cdot \xi|>b\}} g(|u|) d x+g(b) \mathcal{L}^{n}\left(B_{u}^{\xi, a}\right) \\
& \leq \frac{\delta}{2 M} \int_{\Omega} \psi_{0}(|u|) d x+g(b) \mathcal{L}^{n}\left(B_{u}^{\xi, a}\right) \leq \delta
\end{aligned}
$$

Then we define $\mathcal{V}_{\delta}^{\xi}:=\left\{v_{u, b_{\delta}}^{\xi, a_{\delta}}: u \in \mathcal{U}\right\}$, so that condition (a) of Lemma 10.7 is satisfied. 
As for condition (b), we observe that, if $v=v_{u, b_{\delta}}^{\xi, a_{\delta}} \in \mathcal{V}_{\delta}^{\xi}$, then by (11.7) the slices $v_{y}^{\xi}$ satisfy $v_{y}^{\xi}=\sigma_{b_{\delta}}\left(\hat{u}_{y}^{\xi}\right)$ for every $y \in \hat{A}_{u}^{\xi, a_{\delta}}$, and $v_{y}^{\xi}=0$ for every $y \in \hat{B}_{u}^{\xi, a_{\delta}}$. Since, by definition, $\hat{u}_{y}^{\xi} \in \mathrm{BV}(\mathbb{R})$ for every $y \in \hat{A}_{u}^{\xi, a}$, it follows that $v_{y}^{\xi} \in \mathrm{BV}(\mathbb{R})$ and $\left\|v_{y}^{\xi}\right\|_{L^{\infty}(\mathbb{R})} \leq b_{\delta}$ for every $y \in \Pi^{\xi}$. Moreover, $\left|D v_{y}^{\xi}\right|(B) \leq\left|D \hat{u}_{y}^{\xi}\right|(B)$ for every Borel set $B \subset \mathbb{R}$, $J_{v_{y}^{\xi}} \subset J_{\hat{u}_{y}^{\xi}}$, and $\left|\left[v_{y}^{\xi}\right]\right| \leq\left|\left[\hat{u}_{y}^{\xi}\right]\right|$ on $J_{v_{y}^{\xi}}$, hence $J_{v_{y}^{\xi}}^{1} \subset J_{\hat{u}_{y}^{\xi}}^{1}$. Therefore (11.4) implies that

$$
\begin{aligned}
\left|D v_{y}^{\xi}\right|\left(\mathbb{R} \backslash J_{v_{y}^{\xi}}^{1}\right)+\mathcal{H}^{0}\left(J_{v_{y}^{\xi}}^{1}\right) & =\left|D v_{y}^{\xi}\right|\left(\mathbb{R} \backslash J_{\hat{u}_{y}^{\xi}}^{1}\right)+\left|D v_{y}^{\xi}\right|\left(J_{\hat{u}_{y}^{\xi}}^{1} \backslash J_{v_{y}^{\xi}}^{1}\right)+\mathcal{H}^{0}\left(J_{v_{y}^{\xi}}^{1}\right) \\
& \leq\left|D \hat{u}_{y}^{\xi}\right|\left(\mathbb{R} \backslash J_{\hat{u}_{y}^{\xi}}^{1}\right)+\mathcal{H}^{0}\left(J_{\hat{u}_{y}^{\xi}}^{1} \backslash J_{v_{y}^{\xi}}^{1}\right)+\mathcal{H}^{0}\left(J_{v_{y}^{\xi}}^{1}\right) \\
& \leq a_{\delta} \quad \text { for every } y \in \hat{A}_{u}^{\xi, a_{\delta}} .
\end{aligned}
$$

Since $\left|D v_{y}^{\xi}\right|\left(\mathbb{R} \backslash J_{v_{y}^{\xi}}^{1}\right)+\mathcal{H}^{0}\left(J_{v_{y}^{\xi}}^{1}\right)=0$ for every $y \in \hat{B}_{u}^{\xi, a_{\delta}}$, using Lemma 10.8 we obtain (10.20) with $\omega_{\delta}(h):=\left(a_{\delta}+2 a_{\delta} b_{\delta}\right) h$. Therefore condition (b) of Lemma 10.7 is satisfied and the proof of the main assertion is complete.

If (11.3) holds, then $\mathcal{U} \subset L^{1}\left(\Omega ; \mathbb{R}^{n}\right)$ by (11.2) and we can take $g(s)=s$ in the proof, thanks to Remark 10.5. The convergence in $L^{1}\left(\Omega ; \mathbb{R}^{n}\right)$ follows now from the last part of Lemma 10.7.

The following corollary is an easy consequence of Theorem 11.1 and of the arguments used in the proof of Lemma 4.18.

Corollary 11.2. Let $u_{k}$ be a sequence in $\operatorname{GBD}(\Omega)$. Suppose that there exist an increasing continuous function $\psi_{0}: \mathbb{R}^{+} \rightarrow \mathbb{R}^{+}$satisfying (11.1) and a constant $M \in \mathbb{R}^{+}$such that

$$
\int_{\Omega} \psi_{0}\left(\left|u_{k}\right|\right) d x \leq M \quad \text { and } \quad \hat{\mu}_{u_{k}}(\Omega) \leq M
$$

for every $k$, where $\hat{\mu}_{u_{k}}$ is the measure introduced in Definition 4.16. Then there exist a subsequence, still denoted by $u_{k}$, and a function $u \in \operatorname{GBD}(\Omega)$, such that $u_{k} \rightarrow u$ pointwise $\mathcal{L}^{n}$-a.e. on $\Omega$. If, in addition, (11.3) holds, then $u_{k} \in L^{1}\left(\Omega ; \mathbb{R}^{n}\right)$ for every $k$, $u \in L^{1}\left(\Omega ; \mathbb{R}^{n}\right)$, and the subsequence converges strongly in $L^{1}\left(\Omega ; \mathbb{R}^{n}\right)$.

Proof. Since $\hat{\mu}_{u_{k}}^{\xi} \leq \hat{\mu}_{u_{k}}$ for every $\xi \in \mathbb{S}^{n-1}$ and every $k$, by Theorem 11.1 there exist a subsequence, still denoted by $u_{k}$, and an $\mathcal{L}^{n}$-measurable function $u: \Omega \rightarrow \mathbb{R}^{n}$, such that $u_{k} \rightarrow u$ pointwise $\mathcal{L}^{n}$-a.e. on $\Omega$. We want to prove that $u \in \operatorname{GBD}(\Omega)$.

By (11.9) there exist a subsequence, still denoted by $\hat{\mu}_{u_{k}}$, and a measure $\lambda \in \mathcal{M}_{b}^{+}(\Omega)$, such that $\hat{\mu}_{u_{k}} \rightarrow \lambda$ weakly* in $\mathcal{M}_{b}(\Omega)$. By Theorem 3.5 and Definitions 4.8, 4.10, and 4.16 for every $\tau \in \mathcal{T}$, every $\xi \in \mathbb{S}^{n-1}$, and every $\varphi \in C_{c}^{1}(\Omega)$ with $|\varphi| \leq 1$ in $\Omega$, we have

$$
\int_{\Omega} \tau\left(u_{k} \cdot \xi\right) \nabla \varphi \cdot \xi d x \leq \int_{\Omega}|\varphi| d\left|D_{\xi}\left(\tau\left(u_{k} \cdot \xi\right)\right)\right| \leq \int_{\Omega}|\varphi| d \hat{\mu}_{u_{k}}^{\xi} \leq \int_{\Omega}|\varphi| d \hat{\mu}_{u_{k}} .
$$

Passing to the limit as $k \rightarrow \infty$ we get

$$
\int_{\Omega} \tau(u \cdot \xi) \nabla \varphi \cdot \xi d x \leq \int_{\Omega}|\varphi| d \lambda
$$


This implies that for every $\xi \in \mathbb{S}^{n-1}$ and every $\tau \in \mathcal{T}$ the partial derivative $D_{\xi}(\tau(u \cdot \xi))$ belongs to $\mathcal{M}_{b}(\Omega)$ and its total variation satisfies $\left|D_{\xi}(\tau(u \cdot \xi))\right|(B) \leq \lambda(B)$ for every Borel set $B \subset \Omega$. Therefore $u$ satisfies condition (a) of Definition 4.1 for every $\xi \in \mathbb{S}^{n-1}$, hence $u \in \operatorname{GBD}(\Omega)$.

If (11.3) holds, then $u_{k} \in L^{1}\left(\Omega ; \mathbb{R}^{n}\right)$ for every $k$ by (11.9). The other assertions follow from the last part of Theorem 11.1.

We are now in a position to prove the compactness result for $\operatorname{GSBD}(\Omega)$.

Theorem 11.3. Let $u_{k}$ be a sequence in $\operatorname{GSBD}(\Omega)$. Suppose that there exist a constant $M \in \mathbb{R}^{+}$and two increasing continuous functions $\psi_{0}: \mathbb{R}^{+} \rightarrow \mathbb{R}^{+}$and $\psi_{1}: \mathbb{R}^{+} \rightarrow \mathbb{R}^{+}$, with

such that

$$
\lim _{s \rightarrow \infty} \psi_{0}(s)=\infty \text { and } \lim _{s \rightarrow \infty} \frac{\psi_{1}(s)}{s}=\infty
$$

$$
\int_{\Omega} \psi_{0}\left(\left|u_{k}\right|\right) d x+\int_{\Omega} \psi_{1}\left(\left|\mathcal{E} u_{k}\right|\right) d x+\mathcal{H}^{n-1}\left(J_{u_{k}}\right) \leq M
$$

for every $k$. Then there exist a subsequence, still denoted by $u_{k}$, and a function $u \in$ $\operatorname{GSBD}(\Omega)$, such that

$$
\begin{gathered}
u_{k} \rightarrow u \quad \text { pointwise } \mathcal{L}^{n} \text {-a.e. on } \Omega, \\
\mathcal{E} u_{k} \rightarrow \mathcal{E} u \quad \text { weakly in } L^{1}\left(\Omega ; \mathbb{M}_{\mathrm{sym}}^{n \times n}\right), \\
\mathcal{H}^{n-1}\left(J_{u}\right) \leq \liminf _{k \rightarrow \infty} \mathcal{H}^{n-1}\left(J_{u_{k}}\right) .
\end{gathered}
$$

If, in addition, (11.3) holds, then $u_{k} \in L^{1}\left(\Omega ; \mathbb{R}^{n}\right)$ for every $k, u \in L^{1}\left(\Omega ; \mathbb{R}^{n}\right)$, and the subsequence converges strongly in $L^{1}\left(\Omega ; \mathbb{R}^{n}\right)$.

Proof. By (11.10) and (11.11) there exists a constant $M_{1} \in \mathbb{R}^{+}$such that

$$
\int_{\Omega}\left|\mathcal{E} u_{k}\right| d x+\mathcal{H}^{n-1}\left(J_{u_{k}}\right) \leq M_{1}
$$

for every $k$. Therefore (9.19) implies that $\hat{\mu}_{u_{k}}(\Omega) \leq M_{1}$ for every $k$. By Corollary 11.2 there exist a subsequence, still denoted by $u_{k}$, and a function $u \in \operatorname{GBD}(\Omega)$, such that $u_{k} \rightarrow u$ pointwise $\mathcal{L}^{n}$-a.e. on $\Omega$.

Taking into account Remark 4.3, to prove that $u \in \operatorname{GSBD}(\Omega)$ it is enough to show that for $\mathcal{H}^{n-1}$-a.e. $y \in \Pi^{\xi}$ the function $\hat{u}_{y}^{\xi}:=u_{y}^{\xi} \cdot \xi$ belongs to $\operatorname{GSBV}\left(\Omega_{y}^{\xi}\right)$. This property, as well as (11.12)-(11.14), can be obtained as in the proof of [9, Theorem 1.1]. We have just to redefine the function $\mathrm{II}_{y, \xi}\left(u_{k}\right)$ introduced on page 342 of that paper by

$$
\mathrm{II}_{y, \xi}\left(u_{k}\right):=\int_{\Omega_{y}^{\xi}} \psi_{0}\left(\left|\left(\hat{u}_{k}\right)_{y}^{\xi}\right|\right) d t,
$$

and to modify the proof accordingly. In particular we cannot use the boundedness in $\operatorname{BD}(\Omega)$ and we apply the compactness theorem for $\operatorname{GSBV}\left(\Omega_{y}^{\xi}\right)$ (see [6, Theorem 4.36]) to the one-dimensional slices in order to obtain (2.13) and the formula after (2.18) of [9].

If (11.3) holds, the assertions at the end of the theorem follow from the last part of Corollary 11.2. 


\section{Two examples}

In this section we give two examples that show that the compactness result for $\operatorname{GSBD}(\Omega)$ (Theorem 11.3) cannot be easily improved. The first example shows that we cannot expect convergence in $L^{1}\left(\Omega ; \mathbb{R}^{n}\right)$ if we remove (11.3). More precisely, we show that, if we take $\psi_{0}(s)=s$ for every $s \in \mathbb{R}^{+}$, then, in general, we have only pointwise $\mathcal{L}^{n}$-a.e. convergence. Note that in this case (11.11) gives that $u_{k}$ is bounded in $L^{1}\left(\Omega ; \mathbb{R}^{n}\right)$ and that the pointwise limit $u$ belongs to $L^{1}\left(\Omega ; \mathbb{R}^{n}\right)$ by the Fatou lemma.

Example 12.1. Let $x_{0} \in \Omega$, let $\xi \in \mathbb{S}^{n-1}$, and, for every $k$, let $u_{k} \in \operatorname{SBV}\left(\Omega ; \mathbb{R}^{n}\right)$ be defined by $u_{k}(x)=k^{n} \xi$ for $\left|x-x_{0}\right|<1 / k$ and $u_{k}(x)=0$ for $\left|x-x_{0}\right| \geq 1 / k$. Then $\mathcal{E} u_{k}=0 \mathcal{L}^{n}$-a.e. on $\Omega, \mathcal{H}^{n-1}\left(J_{u_{k}}\right)=n \omega_{n} / k^{n-1}$, and $\int_{\Omega}\left|u_{k}\right| d x=\omega_{n}$ for $k$ large enough. Therefore the hypotheses of Theorem 11.3 are satisfied with $\psi_{0}(s):=s$ and $\psi_{1}(s):=s^{2}$ for every $s \in \mathbb{R}^{+}$. The sequence $u_{k}$ converges to 0 pointwise $\mathcal{L}^{n}$-a.e. on $\Omega$, but $u_{k}$ does not converge to 0 in $L^{1}\left(\Omega ; \mathbb{R}^{n}\right)$.

In the rest of this section we construct a sequence $u_{j}$ in $\operatorname{SBD}(\Omega)$ that satisfies all hypotheses of the compactness result for $\operatorname{GSBD}(\Omega)$ (Theorem 11.3), but the limit function $u$ does not belong to $\operatorname{BD}(\Omega)$. Since $u \in \operatorname{GSBD}(\Omega)$, this shows also that $\operatorname{GSBD}(\Omega) \neq$ $\operatorname{SBD}(\Omega)$ and $\operatorname{GBD}(\Omega) \neq \operatorname{BD}(\Omega)$. For the construction we need the elementary result contained in the following lemma.

Lemma 12.2. Let $\rho_{k}$ be a sequence in $\mathbb{R}^{+}$such that

$$
\sum_{k=1}^{\infty} \rho_{k}^{n}<\infty
$$

Then there exists a sequence $x_{k}$ in $\mathbb{R}^{n}$ converging to 0 such that the balls $B_{\rho_{k}}\left(x_{k}\right)$ are pairwise disjoint.

Proof. It is not restrictive to assume that $0<\rho_{k+1} \leq \rho_{k} \leq 1 / 2$ for every $k$. For every integer $i \geq 1$ let $k_{i}$ be the smallest index $k$ such that $\rho_{k} \leq 2^{-i-1}$. Then $k_{1}=1$ and for every $k_{i} \leq k<k_{i+1}$ we have

$$
2^{-i-1}<2 \rho_{k} \leq 2^{-i}
$$

which, together with (12.1), gives

$$
\sum_{i=1}^{\infty}\left(k_{i+1}-k_{i}\right) 2^{-n i}<\infty .
$$

Since $\rho_{k}>0$ for every $k$, we have

$$
\lim _{i \rightarrow \infty} k_{i}=\infty
$$

Let $a_{i}$ be the largest integer such that $\left(k_{i+1}-k_{i}+1\right) 2^{-n i} \leq 2^{-n a_{i}}$. By definition we have

$$
a_{i} \leq i \quad \text { and } \quad 2^{-n a_{i}-n}<\left(k_{i+1}-k_{i}+1\right) 2^{-n i} \leq 2^{-n a_{i}} .
$$


By (12.3) and (12.5) we have

$$
\sum_{i=1}^{\infty} 2^{-n a_{i}}<\infty
$$

Let

$$
\beta_{j}:=\sum_{i=j}^{\infty} 2^{-n a_{i}} .
$$

By (12.6) the sequence $\beta_{j}$ is decreasing and tends to 0 as $j \rightarrow \infty$. Let $j_{0}$ be the largest integer such that $\beta_{1} \leq 2^{-n j_{0}}$. For every integer $j \geq j_{0}$ let $m_{j}$ be the smallest integer $i$ such that $\beta_{i} \leq 2^{-n j}$. Then $m_{j_{0}}=1$ and for every $m_{j} \leq i<m_{j+1}$ we have

$$
2^{-n j-n}<\beta_{i} \leq 2^{-n j}
$$

Since $\beta_{i}>0$ for every $i$, we have

$$
\lim _{j \rightarrow \infty} m_{j}=\infty .
$$

Moreover by (12.7) and (12.8)

$$
\sum_{m_{j} \leq i<m_{j+1}} 2^{-n a_{i}}<\beta_{m_{j}} \leq 2^{-n j} .
$$

If $i \geq m_{j}$, by (12.7) we have

$$
2^{-n a_{i}}<\beta_{i} \leq \beta_{m_{j}} \leq 2^{-n j}
$$

so that by (12.5),

$$
j<a_{i} \leq i .
$$

Let $Q=[0,1)^{n}$ and let $Q_{j}:=2^{-j} Q$. By (12.12) for $m_{j} \leq i<m_{j+1}$ the set $Q_{j-1} \backslash Q_{j}$ is the union of disjoint cubes of the form $z+Q_{i}$, where $z \in 2^{-i} \mathbb{Z}^{n}$ and $\mathbb{Z}$ is the set of integers. We start with $i=m_{j}$ and observe that $\left(k_{i}-k_{i+1}\right) 2^{-n i}<2^{-n a_{i}}<$ $2^{-n j} \leq 2^{-n(j-1)}-2^{-n j}=\mathcal{L}^{n}\left(Q_{j-1} \backslash Q_{j}\right)$ by (12.5) and (12.11). Therefore we can find a family $Q_{k}^{j, m_{j}}, k_{m_{j}} \leq k<k_{m_{j}+1}$, of pairwise disjoint cubes of the form described above and contained in $Q_{j-1} \backslash Q_{j}$. Suppose now that $i=m_{j}+1<m_{j+1}$ and let $A$ be the union (with respect to $k$ ) of the cubes $Q_{k}^{j, m_{j}}, k_{m_{j}} \leq k<k_{m_{j}+1}$. By (12.5) we have $\mathcal{L}^{n}(A)<2^{-n a_{m_{j}}}$, so that $\mathcal{L}^{n}\left(\left(Q_{j-1} \backslash Q_{j}\right) \backslash A\right)>2^{-n j}\left(2^{n}-1\right)-2^{-n a_{m_{j}}} \geq 2^{-n a_{i}}$ by (12.10). Since the set $\left(Q_{j-1} \backslash Q_{j}\right) \backslash A$ is the union of disjoint cubes of the form $z+Q_{i}$, where $z \in 2^{-i} \mathbb{Z}^{n}$ and $i=m_{j}+1$, there exists a family $Q_{k}^{j, i}, k_{i} \leq k<k_{i+1}$, of pairwise disjoint cubes of this form and contained in $\left(Q_{j-1} \backslash Q_{j}\right) \backslash A$. Continuing in the same way for every $m_{j} \leq i<m_{j+1}$, we construct a family $Q_{k}^{j, i}, k_{i} \leq k<k_{i+1}$, of pairwise disjoint cubes of side $2^{-i}$ and contained in $Q_{j-1} \backslash Q_{j}$, such that the cubes of two different families have empty intersection. 
Let $x_{k}^{j, i}, j \geq j_{0}, m_{j} \leq i<m_{j+1}, k_{i} \leq k<k_{i+1}$, be the centres of the cubes $Q_{k}^{j, i}$. By (12.2) we have

$$
B_{\rho_{k}}\left(x_{k}^{j, i}\right) \subset Q_{k}^{j, i}
$$

Let $x_{k}$ be the sequence defined by $x_{k}:=x_{k}^{j, i}$ for $k_{i} \leq k<k_{i+1}, m_{j} \leq i<m_{j+1}$, and $j \geq j_{0}$. By (12.4) and (12.9) we have $x_{k} \rightarrow 0$. Since the cubes $Q_{k}^{j, i}$ are pairwise disjoint, the balls $B_{\rho_{k}}\left(x_{k}\right)$ are pairwise disjoint by (12.13).

The following example shows that $\operatorname{GSBD}(\Omega) \neq \operatorname{SBD}(\Omega)$ and $\operatorname{GBD}(\Omega) \neq \operatorname{BD}(\Omega)$. Moreover it shows that, if a sequence in $\operatorname{SBD}(\Omega)$ satisfies the assumptions of Theorem 11.3, but does not satisfy the assumptions of [9, Theorem 1.1], then the limit of a subsequence may not belong to $\operatorname{SBD}(\Omega)$.

Example 12.3. Assume that $n \geq 2$. Let $p>1$ and let $\rho_{k}$ be a sequence of positive real numbers such that

$$
\sum_{k=1}^{\infty} \rho_{k}^{n-1}<\infty \quad \text { and } \quad \sum_{k=1}^{\infty} \rho_{k}^{n-1-1 / p}=\infty
$$

for instance $\rho_{k}:=k^{-1 /(n-1-1 / p)}$. By Lemma 12.2 there exist a sequence $x_{k}$ and a point $x_{0} \in \Omega$ such that the balls $B_{k}:=B_{\rho_{k}}\left(x_{k}\right)$ are pairwise disjoint and $x_{k} \rightarrow x_{0}$ as $k \rightarrow \infty$. Let $A_{k}$ be a sequence of antisymmetric $n \times n$ matrices such that

$$
\left|A_{k}\right|=\rho_{k}^{-1-1 / p}
$$

for every $k$. From (12.14) and (12.15) we obtain

$$
\sum_{k=1}^{\infty}\left|A_{k}\right|^{p} \rho_{k}^{p+n}<\infty \text { and } \sum_{k=1}^{\infty}\left|A_{k}\right| \rho_{k}^{n}=\infty .
$$

For every $k$ let $w_{k}$ be the function defined by $w_{k}(x)=A_{k}\left(x-x_{k}\right)$ if $x \in B_{k}$ and $w_{k}(x)=0$ if $x \in \Omega \backslash B_{k}$. Finally, let

$$
u_{j}:=\sum_{k=1}^{j} w_{k} \quad \text { and } \quad u:=\sum_{k=1}^{\infty} w_{k} .
$$

For every $j$ the function $u_{j}$ belongs to $\operatorname{SBV}\left(\Omega ; \mathbb{R}^{n}\right) \subset \operatorname{SBD}(\Omega) \subset \operatorname{GSBD}(\Omega)$. Moreover $u_{j} \in L^{\infty}\left(\Omega ; \mathbb{R}^{n}\right)$ and $\mathcal{E} u_{j}=0 \mathcal{L}^{n}$-a.e. in $\Omega$, since each matrix $A_{k}$ is antisymmetric. As $\left|w_{k}\right| \leq\left|A_{k}\right| \rho_{k}$, using the inequalities in (12.14) and (12.16) we find a constant $M \in \mathbb{R}^{+}$ such that (11.11) holds with $\psi_{0}(s)=\psi_{1}(s):=s^{p}$. The inequality in (12.16) implies also that $u \in L^{p}\left(\Omega ; \mathbb{R}^{n}\right)$ and $u_{j} \rightarrow u$ strongly in $L^{p}\left(\Omega ; \mathbb{R}^{n}\right)$ as $j \rightarrow \infty$. By Theorem 11.3 we have $u \in \operatorname{GSBD}(\Omega)$. This follows also from condition (b) of Definition 4.1, using the 
fact that for every Borel set $B \subset \Omega$, every $\xi \in \mathbb{S}^{n-1}$, and $\mathcal{H}^{n-1}$-a.e. $y \in \Pi^{\xi}$ we have $\left|D \hat{u}_{y}^{\xi}\right|\left(B_{y}^{\xi} \backslash J_{\hat{u}_{y}^{\xi}}\right)=0$ and

$$
\begin{aligned}
\int_{\Pi^{\xi}}\left(\left|D \hat{u}_{y}^{\xi}\right|\left(B_{y}^{\xi} \cap J_{\hat{u}_{y}^{\xi}} \backslash J_{\hat{u}_{y}^{\xi}}^{1}\right)+\mathcal{H}^{0}\left(B_{y}^{\xi} \cap J_{\hat{u}_{y}^{\xi}}^{1}\right)\right) d \mathcal{H}^{n-1}(y) \\
\quad \leq \int_{\Pi^{\xi}} \mathcal{H}^{0}\left(B_{y}^{\xi} \cap J_{\hat{u}_{y}^{\xi}}\right) d \mathcal{H}^{n-1}(y) \leq \sum_{k=1}^{\infty} \int_{\Pi^{\xi}} \mathcal{H}^{0}\left(B_{y}^{\xi} \cap\left(\partial B_{k}\right)_{y}^{\xi}\right) d \mathcal{H}^{n-1}(y) \\
\leq \sum_{k=1}^{\infty} \mathcal{H}^{n-1}\left(B \cap \partial B_{k}\right)=: \lambda(B)<\infty
\end{aligned}
$$

where the last inequality follows from (12.14).

Let $E u$ be the matrix-valued Radon measure considered in Remark 4.5. For every $\varepsilon>0$ we have

$$
|E u|\left(\Omega \backslash B_{\varepsilon}\left(x_{0}\right)\right) \geq \frac{1}{\sqrt{2}} \sum_{k=1}^{k_{\varepsilon}} \int_{B_{k}}\left|A_{k}\left(x-x_{k}\right)\right| d \mathcal{H}^{n-1}(x) \geq c_{n} \sum_{k=1}^{k_{\varepsilon}}\left|A_{k}\right| \rho_{k}^{n},
$$

where $k_{\varepsilon}$ is the largest index such that $B_{k} \cap B_{\varepsilon}\left(x_{0}\right)=\emptyset$ for every $k \leq k_{\varepsilon}$, and $c_{n}$ is a constant depending only on the dimension $n$. If $u \in \operatorname{BD}(\Omega)$, then $|E u|\left(\Omega \backslash B_{\varepsilon}\left(x_{0}\right)\right) \leq$ $|E u|(\Omega)<\infty$ for every $\varepsilon>0$. By (12.17) this contradicts the equality in (12.16). Therefore $u \notin \mathrm{BD}(\Omega)$.

Acknowledgments. This material is based on work supported by the Italian Ministry of Education, University, and Research under the Project "Variational Problems with Multiple Scales" 2008 and by the European Research Council under Grant No. 290888 "Quasistatic and Dynamic Evolution Problems in Plasticity and Fracture". I wish to thank Antonin Chambolle for a useful discussion during the Oberwolfach Workshop "Variational Methods for Evolution", December 4-10, 2011. The outcome of this discussion is the second part of Proposition 4.17, which simplifies the proofs in Section 7. I also wish to thank the Mathematisches Forschungsinstitut Oberwolfach for the hospitality on the occasion of that workshop, where this result was first presented.

\section{References}

[1] Alberti, G., Bouchitté, G., Seppecher, P.: Phase transition with line-tension effect. Arch. Ration. Mech. Anal. 144, 1-46 (1998) Zbl 0915.76093 MR 1657316

[2] Ambrosio, L.: A compactness theorem for a new class of functions of bounded variation. Boll. Un. Mat. Ital. B (7) 3, 857-881(1989) Zbl 0767.49001 MR 1032614

[3] Ambrosio, L.: Existence theory for a new class of variational problems. Arch. Ration. Mech. Anal. 111, 291-322 (1990) Zbl 0711.49064 MR 1068374

[4] Ambrosio, L.: A new proof of the SBV compactness theorem. Calc. Var. Partial Differential Equations 3, 127-137 (1995) Z Zbl 0837.49011 MR 1384840

[5] Ambrosio, L., Coscia, A., Dal Maso, G.: Fine properties of functions with bounded deformation. Arch. Ration. Mech. Anal. 139, 201-238 (1997) Zbl 0890.49019 MR 1480240 
[6] Ambrosio, L., Fusco, N., Pallara, D.: Functions of Bounded Variation and Free Discontinuity Problems. Oxford Univ. Press, Oxford (2000) Zbl 0957.49001 MR 1857292

[7] Anzellotti, G.: On the extremal stress and displacement in Hencky plasticity. Duke Math. J. 51, 133-147 (1984) Zbl 0548.73022 MR 0744291

[8] Anzellotti, G., Giaquinta, M.: On the existence of the field of stresses and displacements for an elasto-perfectly plastic body in static equilibrium. J. Math. Pures Appl. 61, 219-244 (1982) Zbl 0467.73044 MR 0690394

[9] Bellettini, G., Coscia, A., Dal Maso, G.: Compactness and lower semicontinuity properties in $\operatorname{SBD}(\Omega)$. Math. Z. 228, 337-351 (1998) Zbl 0914.46007 MR 1630504

[10] Bonetti, E., Frémond, M.: Collisions and fractures: a model in SBD. Rend. Lincei Mat. Appl. 15, 47-57 (2004) Zbl 1072.74004 MR 2102749

[11] Bourdin, B.: Numerical implementation of the variational formulation for quasi-static brittle fracture. Interfaces Free Bound. 9, 411-430 (2007) Zbl 1130.74040 MR 2341850

[12] Bourdin, B., Francfort, G. A., Marigo, J. J.: The variational approach to fracture. J. Elasticity 91, 5-148 (2008) Zbl 1176.74018 MR 2390547

[13] Chambolle, A.: An approximation result for special functions with bounded deformation. J. Math. Pures Appl. (9) 83, 929-954 (2004) Zbl 1084.49038 MR 2112874

[14] Cohn, D. L.: Measure Theory. Birkhäuser, Boston (1980) Zbl 0436.28001 MR 0578344

[15] Dal Maso, G.: An Introduction to $\Gamma$-Convergence, Birkhäuser, Boston (1993) Zbl 0816.49001 MR 1201152

[16] Dal Maso, G., Francfort, G. A., Toader R.: Quasistatic crack growth in nonlinear elasticity. Arch. Ration. Mech. Anal. 176, 165-225 (2005) 1064.74150 MR 2186036

[17] Dal Maso, G., Lazzaroni, G.: Quasistatic crack growth in finite elasticity with noninterpenetration. Ann. Inst. H. Poincaré Anal. Non Linéaire 27, 257-290 (2010) Zbl 1188.35205 MR 2580510

[18] Evans, L. C., Gariepy, R. F.: Measure Theory and Fine Properties of Functions. CRC Press, Boca Raton (1992) Zbl 0804.28001 MR 1158660

[19] Federer, H.: Geometric Measure Theory. Springer, Berlin (1969) Zbl 0176.00801 MR 0257325

[20] Francfort, G. A., Larsen, C. J.: Existence and convergence for quasi-static evolution in brittle fracture. Comm. Pure Appl. Math. 56, 1465-1500 (2003) Zbl 1068.74056 MR 1988896

[21] Francfort, G. A., Marigo, J.-J.: Revisiting brittle fracture as an energy minimization problem. J. Mech. Phys. Solids 46, 1319-1342 (1998) Zbl 0966.74060 MR 1633984

[22] Johnson, C.: Existence theorems for plasticity problems. J. Math. Pures Appl. 55, 431-444 (1976) Zbl 0351.73049 MR 0438867

[23] Kohn, R. V.: New estimates for deformations in terms of their strains. Ph.D. Thesis, Princeton Univ. (1979) MR 2630218

[24] Kohn, R. V., Temam, R.: Dual spaces of stresses and strains, with applications to Hencky plasticity. Appl. Math. Optim. 10, 1-35 (1983) Zbl 0532.73039 MR 0701898

[25] Matthies, H., Strang, G., Christiansen, E.: The saddle point of a differential program. In: Energy Methods in Finite Element Analysis, R. Glowinski et al. (eds.), Wiley, New York, 309-318 (1979) MR 0537013

[26] Miranda, M.: Superfici cartesiane generalizzate ed insiemi di perimetro localmente finito sui prodotti cartesiani. Ann. Scuola Norm. Sup. Pisa Cl. Sci. (3) 18, 515-542 (1964) Zbl 0152.24402 MR 0174706

[27] Percivale, D., Tomarelli, F.: From SBD to SBH: the elastic-plastic plate. Interfaces Free Bound. 4, 137-165 (2002) Zbl 0998.35057 MR 1950527 
[28] Percivale, D., Tomarelli, F.: From special bounded deformation to special bounded Hessian: the elastic-plastic beam. Math. Models Methods Appl. Sci. 15, 1009-1058 (2005) Zbl 1088.49009 MR 2151797

[29] Suquet, P.: Sur les équations de la plasticité: existence et régularité des solutions. J. Mécanique 20, 3-39 (1981) Zbl 0474.73030 MR 0618942

[30] Temam, R.: Mathematical Problems in Plasticity. Gauthier-Villars, Paris (1985) Zbl 0457.73017 MR 0711964

[31] Temam, R., Strang, G.: Functions of bounded deformation. Arch. Ration. Mech. Anal. 75, 7-21 (1980) Zbl 0472.73031 MR 0592100

[32] Temam, R., Strang, G.: Duality and relaxation in the variational problem of plasticity. J. Mécanique 19, 493-527 (1980) Zbl 0465.73033 MR 0595981

[33] Vol'pert, A. I.: Spaces BV and quasilinear equations. Mat. Sb. 73, 255-302 (1967) (in Russian) MR 0216338 